\title{
The modulation of synaptic transmission at the calyx of Held synapse
}

\author{
Dissertation
}

for the award of the degree

'Doctor of Philosophy' (Ph.D.)

in the Sensory and Motor neuroscience Program

of the Göttingen graduate school for neurosciences and molecular biosciences (GGNB)

at the Georg-August-University Göttingen

Faculty of Biology

\section{Submitted by}

Lijun Yao

From Hangzhou, China

\section{Göttingen 2010}


Dr. Takeshi Sakaba Member of the Thesis Committee (Reviewer)

Research group for biophysics of synaptic transmission at the Max Planck Institute for Biophysical Chemistry

Prof. Tobias Moser Member of the Thesis Committee (Reviewer)

Dept. of Otorhynolaryngology, Georg-August-University Göttingen

Prof. Andreas Stumpner Member of the Thesis Committee

Johann-Friedrich-Blumenbach Institute for Zoology and Anthropology, Georg-AugustUniversity Göttingen

Additional reviewers:

Prof. Erwin Neher Max-Planck Institute for Biophysical Chemistry, Göttingen

Prof. Walter Stühmer Max-Planck Institute of Experimental Medicine, Göttingen

Dr. Oliver M. Schlüter European Neuroscience Institute, Göttingen

Date of the thesis defense: at 10am $-11: 30 \mathrm{am}$, on $28^{\text {th }}$ Sep. 2010 


\section{Declaration}

I hereby declare that my $\mathrm{PhD}$ thesis 'The modulation of synaptic transmission at the calyx of Held synapse' has been written independently with no other aids or sources than quoted.

Göttingen, August $31^{\text {th }} 2010$ 


\section{Contents}

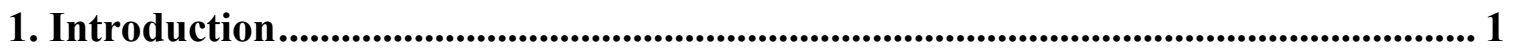

1.1 Neurotransmitter release in the central nervous system................................ 1

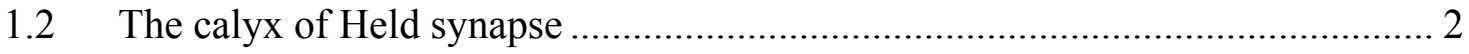

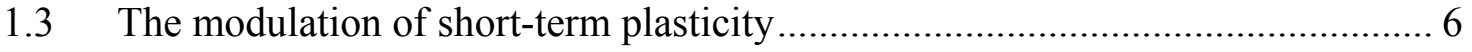

1.4 Ca-dependent vesicle fusion.................................................................. 9

1.5 Membrane retrieval at the calyx of Held synapse .......................................... 13

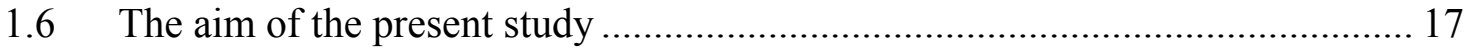

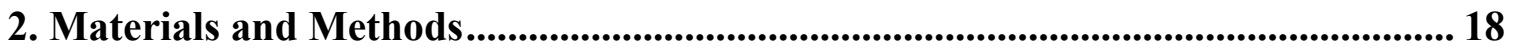

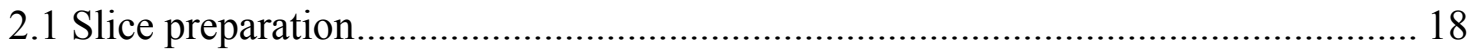

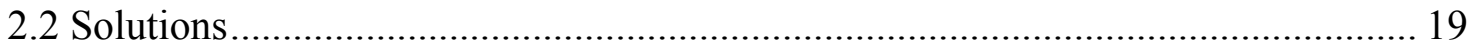

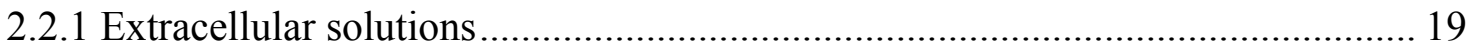

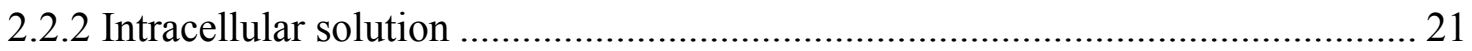

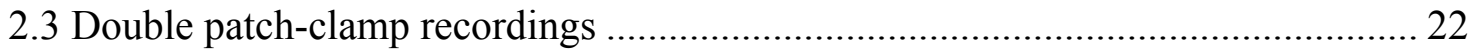

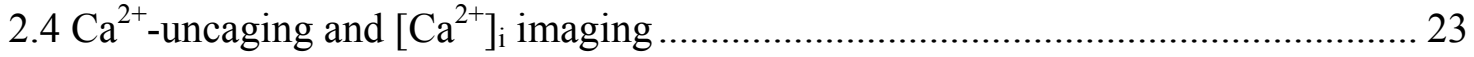

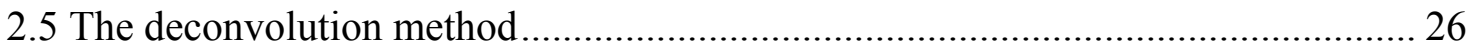

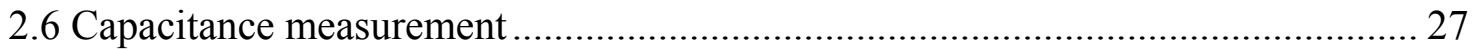

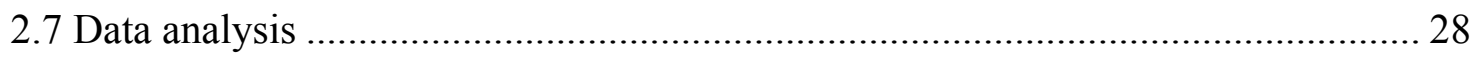

3. Results ......................................................................................................................... 29

3.1 cAMP modulates the intracellular $\mathrm{Ca}^{2+}$ sensitivity of the fast-releasing synaptic

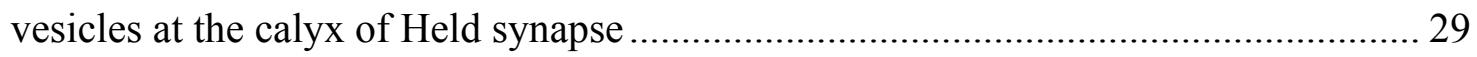

3.1.1 Forskolin increases the synaptic vesicle release at the calyx of Held synapse .... 29

3.1.2 Forskolin increases transmitter release induced by presynaptic $\mathrm{Ca}^{2+}$ uncaging .. 33

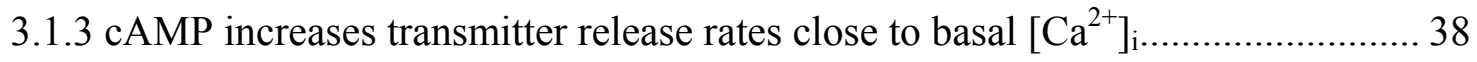

3.1.4 Fast- but not slowly-releasing vesicles are modulated by cAMP ..................... 40

3.1.5 Modelling the $\mathrm{Ca}^{2+}$-dependent synaptic vesicle fusion with a simplified allosteric

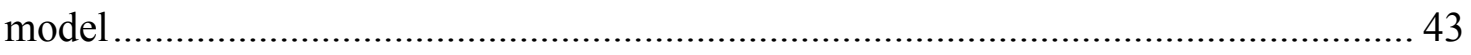

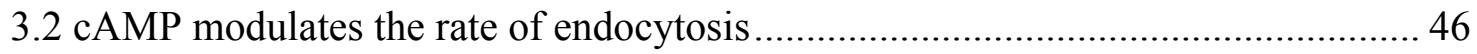

3.2.1 Normal endocytosis at the calyx of Held synapse......................................... 46 
3.2.2 Endogenous cAMP is essential for endocytosis following strong stimulation .... 49

3.2.3 The effect of calmodulin inhibitors on endocytosis at the calyx of Held synapse54

3.2.4 cAMP/PKA is the downstream target of calmodulin during endocytosis

3.2.5 Calmodulin modulates synaptic vesicle replenishment and endocytosis differently

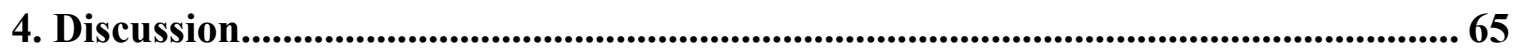

4.1 The potentiation of synaptic transmission by cAMP at the calyx of Held synapse 67

4.2 The underlying mechanism that cAMP increases the intracellular $\mathrm{Ca}^{2+}$ sensitivity of

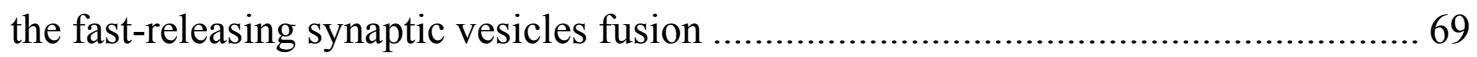

4.3 Calmodulin modulates the rate of endocytosis in response to strong stimulation .. 71

$4.4 \mathrm{cAMP} / \mathrm{PKA}$ is a downstream target after the activation of $\mathrm{Ca}^{2+} /$ calmodulin......... 73

Summary ................................................................................................................. 75

Bibliography ................................................................................................................... 76

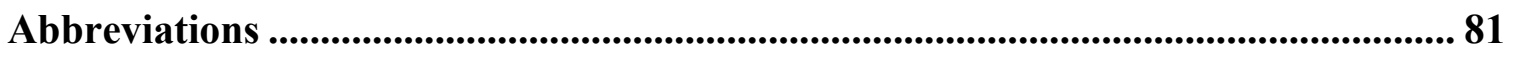

Acknowledgment................................................................................................................ 83

Curriculum Vitae ........................................................................................................................ 84

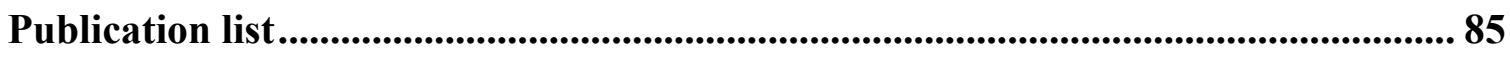




\section{Introduction}

\subsection{Neurotransmitter release in the central nervous system}

In the central nervous system, there are two types of synapses which are responsible for signal transduction between neurons. One is the electrical synapse, where gapjunction channels connect pre- and postsynaptic cells. The second is the chemical synapse (Fig. 1-1); most of neurons use this type of synapses. A chemical synapse can be divided into three parts: presynaptic part, synaptic cleft and postsynaptic part. The presynaptic terminal contains synaptic vesicles, which pack several thousand molecules of neurotransmitters. Some of these vesicles are found to be docked at presynaptic density; named active zone, which contains voltage-gated $\mathrm{Ca}^{2+}$ channels and the proteins relevant for synaptic vesicle fusion. The synaptic cleft usually has a diameter of 20-40 $\mathrm{nm}$, which separates the pre- and post components. The postsynaptic density contains synaptic receptors, which alter the postsynaptic membrane potential and biochemical state upon binding of transmitter molecules.

When an action potential (AP) arrives at a presynaptic terminal, $\mathrm{Ca}^{2+}$ enters the presynaptic terminal through voltage-gated $\mathrm{Ca}^{2+}$ channels. The rise of $\left[\mathrm{Ca}^{2+}\right]_{\mathrm{i}}$ in the terminal triggers synaptic vesicle fusion with the plasma membrane, thereby releasing transmitters into the synaptic cleft. This process is called exocytosis. The released transmitters diffuse into the synaptic cleft and bind to the postsynaptic receptors. The binding of transmitters opens ion channels and changes the membrane conductance and the membrane potential of the postsynaptic cell. This way, the signal is transferred from one neuron to another. The efficiency of synaptic transmission can be modulated either by pre- or postsynaptic factors. At the presynaptic terminal, the number of readilyreleasable vesicles is limited, and the readily-releasable pool (RRP) of vesicles is depleted during a train of APs. In order to maintain steady-state synaptic transmission, the RRP needs to be replenished by new vesicles during the stimulation. Fused vesicles are retrieved by a process called endocytosis. The endocytosed vesicles are recycled and used for the next round of exocytosis. This local recycling of synaptic vesicles is an 
economic way compared with the situation that vesicles have to be transported from the cell body.

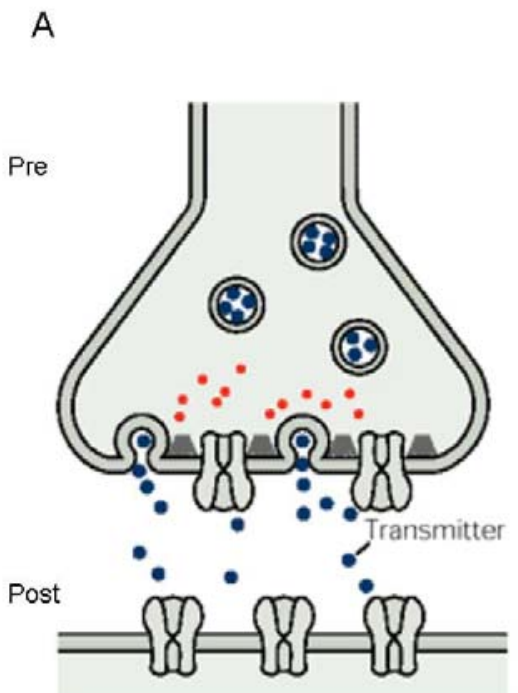

B

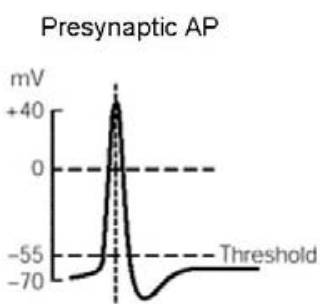

$$
\begin{aligned}
& \text { Excitatory postsynaptic } \\
& \text { potential }
\end{aligned}
$$

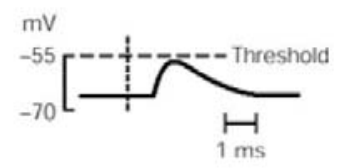

Figure 1-1: Synapse and synaptic transmission in the central nervous system.

A: A simplified diagram of a synapse. B: A presynaptic AP evokes an excitatory postsynaptic potential

\subsection{The calyx of Held synapse}

The calyx of Held is a giant glutamatergic synapse in the mammalian auditory brainstem, which allows direct patch-clamp presynaptic component. In order to study presynaptic function, I chose this synaptic preparation as a model system in my study. The calyx of Held was initially found by Golgi staining in the auditory system in cats, and was named after a German anatomist Hans Held. Later on, Ramón y Cajal used Golgi staining to study the morphology in-depth, and contributed the first high-resolution image of calyx of Held under light-microscope. The location, morphology, properties and the reason for choosing this preparation will be described in this section.

Sound signals arriving at cochlea are transmitted to the ipsilateral anterior cochlear nucleus (aVCN) by auditory nerves onto globular and spherical bushy cells (Fig 1.2). The synapses formed by auditory nerves and spherical bushy cells are named as endbulb of Held. The axons of globular bushy cells then cross the brainstem midline and make contacts with medial nucleus of the trapezoid body (MNTB) in the contralateral 
side. This contact site is called the calyx of Held synapse(Schneggenburger and Forsythe, 2006). Normally, one presynaptic calyx-type terminal makes contact with a single principal cell, but multiple calyces' inputs to one principle cell are also observed with a proportion of $5 \%$ (Bergsman et al., 2004). Also $20 \%$ of the afferent fibers provide two calyces with different principal cells (Kuwabara et al., 1991; Rodriguez-Contreras et al., 2006). Nevertheless, during a paired pre- and postsynaptic recordings, there is only oneto-one relationship in a given synapse. MNTB principal cells not only receive excitatory inputs from calyces, but also receive conventional, small excitatory and inhibitory inputs (Awatramani et al., 2004; Forsythe and Barnes-Davies, 1993; Hamann et al., 2003). The sources of these small inputs are unknown. In some of my experiments, these small inhibitory inputs were blocked with chemical reagents. MNTB principal cells provide inhibitory inputs to the lateral superior olive (LSO). The LSO also receives excitatory inputs from spherical bushy cells of the ipsilateral aVCN. Thus, the signals from both sides converge here. The excitatory input from the ipsilateral side is slightly faster than that from the contralateral side, with a delay of $200 \mu \mathrm{s}$ in cats (Joris and Yin, 1998). So LSO is thought as a detector of binaural signals, and MNTB cells function as a fast, signinverting relay station.

A

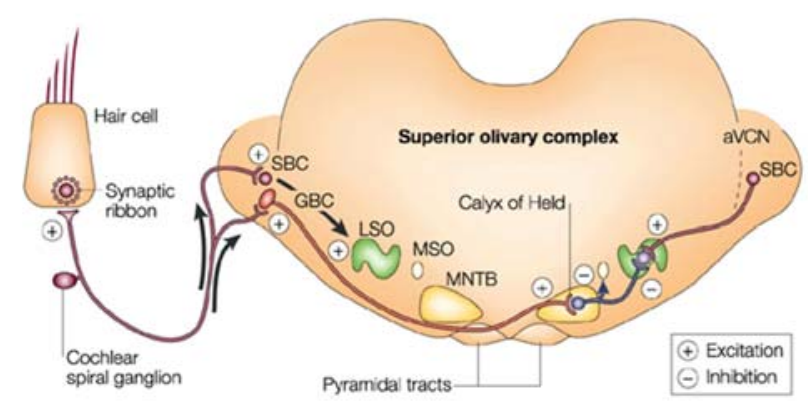

B

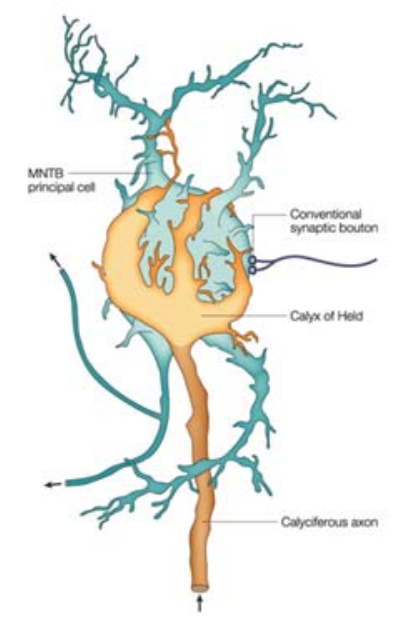

Figure 1-2: the structure and location of the calyx of Held synapse.

A: The calyx of Held synapse in the auditory brain stem circuit. The calyx of Held synapse composes 
of the axon of contralateral globular bushy cells (GBCs) and the cell body of medial nucleus of the trapezoid body (MNTB). Principle cells in MNTB project axons to the ipsilateral leteral superior olive (Washbourne et al.) to form inhibitory synapses. B: Morphology of the calyx of Held synapse; a yellow part is the presynaptic compartment, while a blue part is the pincinple neuron. Modified from von Gersdorff and Borst (2002)

Because of its large size, it is possible to patch-clamp both pre-and post components of the synapse. Direct recordings from the calyx of Held presynaptic terminal were performed in an acute slice preparation of the rat brainstem (Borst et al., 1995; Forsythe, 1994). Using this preparation, one can study pre-synaptic functions such as ion channels, transmitter release and regulation of the $\mathrm{Ca}^{2+}$ signals directly. Also, the accessibility to the presynaptic terminal allows one to manipulate the presynaptic function more easily by introduction of $\mathrm{Ca}^{2+}$ dyes, Ca chelators, peptides, antibodies to the presynaptic terminal through patch pipettes. Therefore, the calyx of Held is a model system to study synaptic functions, and especially presynaptic functions.

We have obtained a lot of insight into the mechanisms of synaptic transmission using the calyx of Held synapse, in the last decade. Under whole-cell voltage-clamp from MNTB neurons, a two-component excitatory postsynaptic currents (EPSCs) was observed: the fast component was mediated by $\alpha$-amino-3-5-methyl-4-isoxazolepropionic acid (AMPA) receptors whereas the slow component was mediated by N-methyl-Daspartate (NMDA) receptors (Forsythe and Barnes-Davies, 1993). After postnatal maturation, only a small fraction of NMDA-EPSCs remained after P20 (Joshi and Wang, 2002; Taschenberger and von Gersdorff, 2000). Due to the slow kinetics and gradually disappearance of the NMDA receptors, I only recorded AMPA receptors mediated EPSC as to monitor presynaptic vesicle fusion. At the same time the miniature EPSCs (mEPSCs) and AMPA receptor-mediated EPSCs become faster with development. The fast AMPA EPSC decay is caused by the GluRD subunits, which intrinsically have fast deactivation and desensitization kinetics (Joshi et al., 2004; Koike-Tani et al., 2005).

The RRP size is an important parameter for a given synapse, which determines the efficiency and fidelity of synaptic transmission. EM reconstruction of a single calyx of Held from P9 rat has shown that each presynaptic terminal contains 550 individual active zones with an average distance of $\sim 0.6 \mu \mathrm{m}$. These active zones are 
morphologically very similar to conventional CNS synapses. Each active zone has an average surface area of $0.1 \mu \mathrm{m}^{2}$, and contains $\sim 2$ docked vesicles (Satzler et al., 2002). Several different protocols have been used to estimate the number of vesicles in the RRP, such as back-extrapolation of the cumulative EPSC amplitude induced by high-frequency stimuli (Bollmann et al., 2000; Schneggenburger et al., 1999), deconvolution of the EPSCs induced either by depolarizing pulses or $\mathrm{Ca}^{2+}$-uncaging (Lou et al., 2005; Neher and Sakaba, 2001a; Sakaba and Neher, 2001c; Wölfel and Schneggenburger, 2003) and presynaptic capacitance measurements (Sun and $\mathrm{Wu}, 2001$ ). Although the number of the vesicle pool is somewhat heterogenous among cells, overall, the RRP consists of $\sim 3000$ 4000 vesicles at the calyx of Held. One AP releases $\sim 150-200$ vesicles, meaning a release probability of $\sim 5-7 \%$ (Schneggenburger and Forsythe, 2006). In this study, I used the above-mentioned techniques, and found that cAMP increases the pool size to some extent. With respect to the $\mathrm{Ca}^{2+}$ influx through voltage-gated $\mathrm{Ca}^{2+}$ channels, $\mathrm{N}-, \mathrm{R}$ - and P-type of $\mathrm{Ca}^{2+}$ channels are expressed before the opening of the auditory canal $(<\mathrm{P} 10)$ (Wu et al., 1999). In contrast, only P-type of $\mathrm{Ca}^{2+}$ channels contributes to $\mathrm{Ca}^{2+}$ influx at mature calyces (P20) (Iwasaki et al., 2000; Iwasaki and Takahashi, 1998).

Although there are a lot of functional differences between the calyx synapse and other small synapses in the nervous system, calyces do share a lot of similarities with conventional CNS synapses such as: each active zone contains 100 200 clear-core vesicles (Satzler et al., 2002); P/Q type of calcium channels is a dominant $\mathrm{Ca}^{2+}$ channel subtype (Iwasaki and Takahashi, 1998; Wu et al., 1999) especially in mature calyces (Iwasaki and Takahashi, 1998); high-frequency presynaptic firing causes short-term depression of the postsynaptic responses (von Gersdorff and Borst, 2002); endocytosis is slow during mild stimulation and becomes fast in response to strong stimulation (Wu et al., 2005). Although the calyx terminal contains unusually high numbers of active zones, overall release probability of the synaptic vesicle is similar to that of conventional boutons (Zucker and Regehr, 2002). Therefore, the calyx of Held could be considered as a cluster of conventional synaptic boutons, and the principle of synaptic transmission should be somewhat conserved. Therefor the results obtained from the calyx synapse may have some relevance to other CNS synapses. 


\subsection{The modulation of short-term plasticity}

The synaptic efficiency can be modified upon patterns of the recent stimulation, displaying enhancement or depression. Short-term plasticity represents increase or decrease of synaptic strengths, which last at most a few minutes. Dependinng on the lifetimes of the effect, short-term synaptic enhancement can be categorized into several forms, such as facilitation, which can last hundreds of milliseconds; augmentation, the effect of which grows and decays with a time constant of 5-10 s; and post-tetanic potentiation, which can be only induced by intense stimulation and lasts $30 \mathrm{~s}$ to several minutes (Zucker and Regehr, 2002). At a single synapse, these three types of enhancement together with synaptic depression can co-exist, and synaptic plasticity depends on the stimulation pattern, the presence of neuromodulators and the development stages. The same stimulation pattern does not necessarily induce the same form of synaptic plasticity at different synapses, and some synapses may show facilitation and others may show strong depression. The input-output relationship between the pre- and postsynaptic neurons can be dynamically modulated by short-term synaptic plasticity, determining how the signal is transformed from one neuron to the other. To understand the cellular and molecular mechanisms underling short-term plasticity is, therefore, pivotal to uncover the process of synaptic transmission.

The mechanisms of short-term plasticity are still not well understood. Modification of synaptic strengths could arise from pre- or postsynaptic factors. Presynaptically, the number of synaptic vesicles in the RRP, their $\mathrm{Ca}^{2+}$ sensitivity for release, and the amplitude of $\left[\mathrm{Ca}^{2+}\right]_{\mathrm{i}}$ near the $\mathrm{Ca}^{2+}$ sensor are important parameters subject to modulation, and hence determine the amount of transmitter release in response to an APs. Postsynaptically, desensitization and saturation of the postsynaptic receptors can affect the efficiency of synaptic transmission. Notably, postsynaptic mechanisms are relatively well understood whereas the presynaptic mechanisms remain unsolved. One reason is that most of the synaptic terminals in the central nervous system are too small to be manipulated by electrophysiogical methods. The calyx of Held synapse provides a large terminal, which allows one to examine presynaptic mechanisms for short-term plasticity using conventional electrophysiology. However, this synapse also has several disadvantages. For example, lack of NMDA receptors especially in mature animals (Futai 
et al., 2001; Taschenberger and von Gersdorff, 2000) makes it impossible to study certain types of plasticity, such as long-term potentiation, induction of which recquires NMDA receptors.

For facilitation, the residual $\mathrm{Ca}^{2+}$ hypothesis is the most prevalent hypothesis (Zucker and Regehr, 2002). The residual $\mathrm{Ca}^{2+}$ refers to the sub-micromolar, bulk $\mathrm{Ca} 2+$, which is caused upon an AP invasion in the presynaptic terminal and decays slowly following the AP. If the next AP comes in a short time, elevation of local $\left[\mathrm{Ca}^{2+}\right]_{\mathrm{i}}$ through $\mathrm{Ca}$ channels is added to the residual $\mathrm{Ca}^{2+}$ from the previous AP, inducing larger EPSCs. The first evidence in support of this hypothesis was obtained from neuromuscular junctions. A preceding AP can cause facilitation of postsynaptic responses to subsequent stimuli even if transmitter release does not occur during the first AP. Therefore presynaptic $\mathrm{Ca}^{2+}$ entry during the first AP is critical for facilitation (Del Castillo and Katz, 1954; Dudel and Kuffler, 1961; Katz and Miledi, 1968). This implies that $\mathrm{Ca}^{2+}$ influx is responsible for facilitation, at least, in some conditions. This hypothesis was confirmed by the observation that presynaptic introduction of a slow Ca buffer EGTA diminished facilitation (Cuttle et al., 1998; Hori and Takahashi, 2009). In the calyx of Held, depression is dominant under physiological condition at immature terminals; facilitation can be seen at reduced initial release probability by lowering the extracellular $\mathrm{Ca}^{2+}$ concentration during trains of fiber stimulation (Barnes-Davies and Forsythe, 1995; Borst et al., 1995). In addition to the residual $\mathrm{Ca}^{2+}$ hypothesis, $\mathrm{Ca}^{2+}$-dependent facilitation of presynaptic calcium currents may also contribute to facilitation of transmitter release at the calyx of Held (Borst and Sakmann, 1998; Cuttle et al., 1998). The increase in the size of RRP and the $\mathrm{Ca}^{2+}$ sensitivity for vesicle fusion do not contribute to facilitation of transmitter release (Felmy et al., 2003; Hori and Takahashi, 2009), but are relevant for potentiation of release in response to activation of second messengers (Kaneko and Takahashi, 2004; Lou et al., 2005; Sakaba and Neher, 2001b).

For synaptic depression, both pre- and postsynaptic mechanisms could be responsible at the calyx of Held synapse. Depletion of RRP is a key element of depression. The magnitude of depression largely depends on the frequency of the stimulation. For P8-P11 calyces, depression is only induced by presynaptic mechanisms when the stimulation frequency is below $10 \mathrm{~Hz}$ (Borst et al., 1995; von Gersdorff et al., 
1997). With the increase of the frequency, depression becomes stronger, especially with a frequency $>100 \mathrm{~Hz}$. Postsynaptic receptor desensitization is also responsible at high frequency. When desensitization and saturation of the postsynaptic receptors are blocked by cyclothiazide and kynurenic acid, depression is attenuated, but still exists, which indicates that presynaptic mechanisms also contribute to depression (Neher and Sakaba, 2001a; Wang and Kaczmarek, 1998; Wu and Borst, 1999). In summary, multiple mechanisms are involved in the process of synaptic facilitation and depression, and modulation of pre- or postsynaptic mechanisms is crucial for the fidelity of the signal transduction.

Besides the frequency and pattern of the stimulation as described above, signaling molecules called second messengers affecting pre- or post synaptic functions and modulate the strength of neural transmission. The second messengers are thought to be activated by presynaptic activity and are involved in the underlying mechanisms of shortterm plasticity induced by nerve stimulation. Usually, they are activated by prolonged stimulation leading to accumulation of $\mathrm{Ca}^{2+}$, which triggers several signaling cascades and changes the synaptic efficacy. Therefore, second mseesngers are postulated to be relevant for augmentation, post-tetanic and long-term potentiation in some synapses. In addition to the nerve activity, second messengers are also activated by neuromodulators, for example, serotonin, dopamine, and peptide transmitters.

cAMP (Cyclic adenosine monophosphate) is one of the best-studied second messengers, used for intracellular signal transduction. It is synthesized from ATP by adenylate cyclase (Sun et al.); cAMP activates multiple protein kinases and the effector proteins. In the chemical synapse, it was reported that cAMP can activate PKA, thereby facilitating transmitter release (Byrne and Kandel, 1996; Capogna et al., 1995; ChavezNoriega and Stevens, 1994; Goy and Kravitz, 1989; Kondo and Marty, 1997; Llano and Gerschenfeld, 1993; Trudeau et al., 1996; Weisskopf et al., 1994). This effect of cAMP is thought to underlie long-term potentiation of synaptic efficacy and memory consolidation (Bailey et al., 1996; Silva et al., 1998). However, PKA-independent actions of cAMP, which facilitate release of transmitters or hormones, have also been reported. For example, in crayfish neuromuscular junction, cAMP can activate presynaptic Ih channels to regulate synaptic plasticity (Beaumont and Zucker, 2000; Ozaki et al., 2000). In 
pancreatic beta cells, cAMP activates Epac through Epac-Rim2 and/or Epac-ryanodine receptor to regulate insulin secretion (Eliasson et al., 2003; Kashima et al., 2001; Ozaki et al., 2000). Epacs are guanine nucleotide exchange factors (GEFs) for Rap1 and Rap2, These proteins are also involved in a number of diseases, neurodegeneration, inflammatory and metabolic disease. In the calyx of Held, Sakaba \& Neher reported that forskolin, an activator of AC, potentiated EPSCs. cAMP selectively increases the number of vesicles with high release probability whereas the slow component of release was not changed (Sakaba and Neher, 2001b). A subsequent paper demonstrated cAMP facilitates transmitter release by increasing both the release probability and number of release vesicles, and this effect is mediated by cAMP-Epac pathway (Kaneko and Takahashi, 2004). The exact mechanisms for cAMP modulation of synaptic transmission still need to be verified, which is one of the main topics of this thesis.

\subsection{Ca-dependent vesicle fusion}

It is well known that $\mathrm{Ca}$ triggers vesicle fusion. The efficiency of synaptic vesicle fusion depends on the amplitude and kinetics of the $\left[\mathrm{Ca}^{2+}\right]_{\mathrm{i}}$ in the presynaptic terminal. When an AP arrives at the terminal, $\left[\mathrm{Ca}^{2+}\right]_{\mathrm{i}}$ rises and drops very rapidly within milliseconds timescale. This fast synchronous transmitter release is sometimes followed by a prolonged, delayed asynchronous release. The transient and localized elevation of $\left[\mathrm{Ca}^{2+}\right]_{\mathrm{i}}$ occurs very close to the open $\mathrm{Ca}^{2+}$ channels, and this spatally-limited increase of the $\mathrm{Ca}^{2+}$ usually is termed as "microdomain $\mathrm{Ca}^{2+}$ ", as opposed to the global $\left[\mathrm{Ca}^{2+}\right]_{\mathrm{i}}$. How high is the microdomain $\left[\mathrm{Ca}^{2+}\right]_{\mathrm{i}}$ during a single AP in a CNS synapse? Initially, theoretical and functional studies postulated that increase of $\left[\mathrm{Ca}^{2+}\right]_{\mathrm{i}}$ to $>100 \mu \mathrm{M}$ triggers synchronous release (Augustine et al., 1991; Llinas et al., 1992; Naraghi and Neher, 1997; Neher, 1998; Yamada and Zucker, 1992). Asynchronous release, in contrast, has been proposed to be mediated by another sensor with higher affinity for $\mathrm{Ca}^{2+}$ because the time course of aynchronous release matches with that of the global $\mathrm{Ca}^{2+}$ (Zucker, 1999).

The precise time course of microdomain $\mathrm{Ca}^{2+}$ during an AP has been difficult to measure due to the spatial and temporal limitation of the existing techniques. Nevertheless, there are several attempts to monitor the microdomain $\mathrm{Ca}^{2+}$, such as fast imaging techniques with low-affinity luminescent $\mathrm{Ca}^{2+}$ indicator, which could detect 
microdomain $\mathrm{Ca}^{2+}$ with a size of $<1 \mu \mathrm{m}$ at the squid giant synapse (Llinas et al., 1995). Total internal reflection fluorescence microscopy shows hot spots close to the plasma membrane in inner hair cells, bipolar neurons and chromaffin cells (Becherer et al., 2003; Zenisek et al., 2003). However, optical imaging even with fastest time resolution cannot monitor the real kinetics of $\left[\mathrm{Ca}^{2+}\right]_{\mathrm{i}}$ because of the reaction speed of the $\mathrm{Ca}^{2+}$ dyes. $\mathrm{Ca}^{2+}$ uncaging is an alternative approach to study the Ca-dependent vesicle fusion. This technique can elevate spatially homogeneous $\left[\mathrm{Ca}^{2+}\right]_{i}$, and therefore, all the synaptic vesicles in the presynaptic terminal "see" the same concentration of $\left[\mathrm{Ca}^{2+}\right]_{\mathrm{i}}$, which can be monitored by conventional $\mathrm{Ca}^{2+}$ fluorimetry. With $\mathrm{Ca}^{2+}$ uncaging, one can measure $\mathrm{Ca}-$ dependent vesicle fusion quantitatively. This technique has been already applied to several preparations, such as chromaffin cells (Heinemann et al., 1994), retina bipolar cells (Heidelberger et al., 1994), photoreceptor ribbon synapses (Kreft et al., 2003; Thoreson et al., 2004) and inner hair cells in the cochlea (Beutner et al., 2001). All these cells have large size, but most of the synapses in the CNS are very small in size. Technically it is rather difficult to introduce the caged $\mathrm{Ca}^{2+}$ compounds to the small terminal via a patch pipette and hence quantitatively study the Ca-dependent vesicle fusion. Taking advantage of the large size of the calyx of Held, it is possible to apply caged compounds via a patch pipette.

Two parallel studies used $\mathrm{Ca}^{2+}$ uncaging to measure the $\mathrm{Ca}^{2+}$-sensitivity of vesicle fusion at this synapse (Bollmann et al., 2000; Schneggenburger and Neher, 2000). The $\mathrm{Ca}^{2+}$ sensitivity for vesicle fusion actually is lower than expected from previous studies and transient elevation of $\left[\mathrm{Ca}^{2+}\right]_{\mathrm{i}}$ to $10-25 \mu \mathrm{M}$ in less than $0.5 \mathrm{~ms}$ timescale triggers transmitter release during an single AP, which can release $10 \%$ of the RRP. So the Ca sensors are not equilibrated with $\mathrm{Ca}^{2+}$ and far from saturation during an AP invasion. This allows strong modulation of synaptic strengths by varying the presynaptic $\mathrm{Ca}^{2+}$ influx and $\mathrm{Ca}^{2+}$ sensitivity. Quantitatively, the $\left[\mathrm{Ca}^{2+}\right]_{\mathrm{i}}$ elevation to $1-2 \mu \mathrm{M}$ triggers spontaneous fusion; and in the range of $2-8 \mu \mathrm{M}$, vesicle release rates are highly dependent on the $\left[\mathrm{Ca}^{2+}\right]_{\mathrm{i}}$, with a slope of 4-5 in the double-logarithmic plot of transmitter release rates (or release rate per vesicle) as a function of $\left[\mathrm{Ca}^{2+}\right]_{\mathrm{i}}$. The non-linearity observed at the calyx synapse confirmed the data of high $\mathrm{Ca}^{2+}$ co-operativity in triggering vesicle release estimated from varying the extracellular $\mathrm{Ca}^{2+}$ concentration (Dodge and 
Rahamimoff, 1967). When the $\left[\mathrm{Ca}^{2+}\right]_{\mathrm{i}}$ was elevated $>10 \mu \mathrm{M}$, the RRP was depleted within a few ms, and even higher $\left[\mathrm{Ca}^{2+}\right]_{\mathrm{i}}$ only accelerates the rise time of the EPSC and shortens the synaptic delay without changing the amplitude of EPSCs. This indicates the depletion of the RRP. A subsequent depolarization after flash photolysis induces no vesicle release, which indicates that the two different types of stimuli act on the same vesicle pool.

When the relationship between peak transmitter release rates and $\left[\mathrm{Ca}^{2+}\right] \mathrm{i}$ are fitted by a release model which assumes sequential binding of $\mathrm{Ca}^{2+}$ to the sensor, five $\mathrm{Ca}^{2+}$ binding steps are required for one vesicle release in order to achieve high $\mathrm{Ca}$ cooperativity (Bollmann et al., 2000; Schneggenburger and Neher, 2000). Although models in these two papers are very similar, some differences are also present such as the mechanism of $\mathrm{Ca}^{2+}$-binding, which assumed there is no (Bollmann et al., 2000) or have (Schneggenburger and Neher, 2000) an intrinsic cooperativity factor. Also, Bollmann et al. (2000) assumed that after binding to five $\mathrm{Ca}^{2+}$, a final reversible $\mathrm{Ca}^{2+}$-independent isomerization step was required to promote vesicle fusion. Nevertheless, both models work well when the elevated $\left[\mathrm{Ca}^{2+}\right]_{\mathrm{i}}$ is above $2 \mu \mathrm{M}$. However according to these models when the $\left[\mathrm{Ca}^{2+}\right]_{\mathrm{i}}$ goes lower toward basal $\left[\mathrm{Ca}^{2+}\right]_{\mathrm{i}}$ level, vesicle release should be stopped. On the other hand, experimentally this is not the case, because of occurrence of spontaneous fusion events at the bsal $\mathrm{Ca}^{2+}$ level, and below $2 \mu \mathrm{M}$ the $\mathrm{Ca}^{2+}$ cooperativity is gradually decreased to the value of 1 . Based on this result, Lou et al. developed an allosteric $\mathrm{Ca}^{2+}$-binding model for vesicle fusion (Lou et al., 2005). In this model, a rate constant $1+$ (also called fusion 'willingness') is added, which allows low rates of vesicle fusion in the absence of bound $\mathrm{Ca}^{2+}$, and higher rates of vesicle fusion are attained when the $\left[\mathrm{Ca}^{2+}\right]_{\mathrm{i}}$ is high. This model can estimate release rates for the whole range of $\left[\mathrm{Ca}^{2+}\right]_{\mathrm{i}}$, and postulates one Ca-sensor can account for all the vesicle fusion.

In vertebrate synapses, synaptotagmin 1, 2 and 9 (Syt1, Syt2 and Syt9) serve as Ca2+ sensors for fast synchronous release (Fernandez-Chacon et al., 2001; Geppert et al., 1994; Pang et al., 2006a; Xu et al., 2007). Calyx of Held synapse only expresses Syt2, and mutation or knockout of Syt2 impairs synchronous release (Pang et al., 2006b; Sun et al., 2007). In Syt2 KO mice, Sun et al. measured remaining asynchronous release at the calyx of Held, and postulated a dual- $\mathrm{Ca}^{2+}$-sensor model (Sun et al., 2007). This model 
assumes that 1) Syt2 is the $\mathrm{Ca}^{2+}$-sensor responsible for synchronous release with $\mathrm{Ca}^{2+}$ cooperativity of $\sim 5 ; 2$ ) another unknown $\mathrm{Ca}^{2+}$-sensor is responsible for asynchronous release with a $\mathrm{Ca}^{2+}$ cooperativity of $\sim 2$; 3) an effectivity factor $\mathrm{C}$ accounts for spontaneous release at $0 \mathrm{Ca}^{2+}$. These three pathways compete with each other to operate on the same synaptic vesicle (Sun et al., 2007). It is unknown if one $\mathrm{Ca}^{2+}$-sensor trigger all synaptic vesicle release events in the whole range of $\mathrm{Ca}^{2+}$, or several sensors are required. In addition, the second sensor responsible for asynchronous /spontaneous fusion remains to be identified. So in my study, allosteric $\mathrm{Ca}^{2+}$-binding model was used to explain the modulation by cAMP. Although two $\mathrm{Ca}^{2+}$-sensor model can fit my data, it is difficult to discriminate which model is correct with our experiments. Therefore I don't aim to distinguish between these two models.

Besides, presynaptic release mechanisms undergo extensive changes during the postnatal maturation of the calyces. From P9-P11 to P16-P19, calyces are remodeled from immature spoon-like structure to the multi-digit morphology (Kandler and Friauf, 1993). The AP waveform become faster and briefer in more mature calyces (Taschenberger and von Gersdorff, 2000), thus the amount of $\mathrm{Ca}^{2+}$ influx is reduced during an AP. EPSCs become larger in mature calyces, which is mainly due to the larger RRP size (approximately twice) (Ishikawa and Takahashi, 2001; Taschenberger and von Gersdorff, 2000). Furthermore, the kinetics of AP evoked release rate become faster in mature calyx (Taschenberger et al., 2005). How less $\mathrm{Ca}^{2+}$ influx induces fast vesicle fusion? Wang $\mathrm{LY}$ et al. applied $\mathrm{Ca}^{2+}$ uncaging in mature calyces to address this question. They found that the dose-response curve between release rate and $\left[\mathrm{Ca}^{2+}\right]_{i}$ was only slightly shifted to the right side at more mature synapses, which means that the intracellular $\mathrm{Ca}^{2+}$ sensitivity for vesicle fusion is decreased. They concluded that the coupling between readily releasable vesicles and $\mathrm{Ca}^{2+}$ channels became tighter during synaptic maturation whereas the $\mathrm{Ca}^{2+}$-sensing mechanism was rather similar (Wang et al., 2008). We do not aim to study the developmental change by cAMP; therefore animals were used between the age of P8 and P11 in this study. 


\subsection{Membrane retrieval at the calyx of Held synapse}

After synaptic vesicles fuse with presynaptic membrane, the expanded membrane undergoes endocytosis in order to maintain the presynaptic structure and transmission intact. So far, modulation of second messengers on transmitter release was described. In addition, second messengers may also modulate the rate of membrane retrieval, thereby regulating the recycling of synaptic vesicles. This may not control the synaptic efficacy immediately, but may have some consequences in the longer time scale. This is another topic of my study. Specifically, the modulation of endocytosis by cAMP was investigated at the calyx of Held.

Generally, depending on the strength and pattern of the stimulation, several different forms of endocytosis are triggered. Each form has different kinetics and may underlie different molecular mechanisms.

1) Slow endocytosis, which takes tens of seconds, is the best studied form of endocytosis. Synaptic vesicles are fully fused with plasma membrane during exocytosis, followed by a clathrin-mediated membrane invagination and fission (Heuser, 1989; Heuser and Reese, 1973). Endocytotic adapters and accessory proteins are necessary for this process (Royle and Lagnado, 2003). In the calyx of Held, slow endocytosis can be induced by mild (such as an AP-like stimulation) (Sun et al., 2002; Wu et al., 2005; Yamashita et al., 2005) and intermediate stimulations (tens of AP-like stimuli or tens of ms of depolarizing pulses) (Hosoi et al., 2009; Wu et al., 2005; Yamashita et al., 2005). The time constant of slow endocytosis is linearly proportional to the amount of exocytosis (Wu et al., 2005). This means that the absolute rate of clathrin-dependent slow endocytosis is the same irrespective of the stimuli strengths, which in other words indicates a limited capacity of endocytosis. Because of the limited capacity of clathrinmediated slow endocytosis, other forms of membrane retrieval are considered to be required for keeping the fidelity of neurotransmission. Slow form of endocytosis is mediated by dynamin at the calyx synapse (Yamashita et al., 2005), which is consistent with the results obtained from other preparations (Koenig and Ikeda, 1989; Newton et al., 2006; Palfrey and Artalejo, 1998).

2) Fast endocytosis, which takes several hundreds of milliseconds to few seconds, and can be induced with massive stimulation, such as hundreds ms of depolarizing pulses 
or hundreds of AP-like stimuli at high frequencies (Wu et al., 2005). This rapid form could reflect "kiss-and-run" retrieval at many small synapses, such as hippocampus synapses (Aravanis et al., 2003; Klyachko and Jackson, 2002). Under the "Kiss-and-run" mode of vesicle cycling, vesicles transiently fuse with plasma membrane and open a fusion pore, releasing a small amount of transmitters, without full collapse of vesicle membrane (Fesce et al., 1994). In the calyx of Held, cell-attached capacitance recordings indicate that only a minor fraction of fusion events arise from "kiss-and-run", and that most of the fusion events are full collapse fusion during the application of high potassium solution (He et al., 2006; Wu et al., 2007). Furthermore, the fast component of endocytosis is not recycled within the RRP but recycled into the recycling pool (Wu and $\mathrm{Wu}, 2009$ ), which is against the view that the fast endocytosed vesicles are relevant for "kiss-and run" events, recycled within the RRP, and are rapidly reused. Wu et al. also suggested that fast endocytosis provides a rapid mechanism of speed in replenishing the recycling vesicles and is particularly important during high frequency firing, in order to maintain the normal structure of the terminal and clearing the fused vesicles from active zones. This high frequency firing, which is strong enough to induce rapid form of endocytosis (von Gersdorff and Borst, 2002), does occur under physiological conditions at calyx of Held and also at other synapses,

3) Bulk endocytosis, is an activity-dependent form of endocytosis with high capacity of membrane retrieval, and it occurs only infrequently. An endosome-like structure appears in the cytosol in a short time after the stimulation, which possibly corresponds to large amounts of membrane retrieved from the plasma membrane (Holt et al., 2003; $\mathrm{Wu}$ and $\mathrm{Wu}, 2007)$. Synaptic vesicles are then generated from endosome-like structure to form new vesicles. Bulk endocytosis has been initially described at the frog neuromuscular junction under nonphysiological massive stimulation such as stimulation with high potassium (Miller and Heuser, 1984). Later on, bulk endocytosis was shown to occur under more physiological conditions in neuromuscular junctions (Richards et al., 2000), cultured cerebellum neurons (Marxen et al., 1999), retinal bipolar neurons (Holt et al., 2003), and also at the calyx of Held synapses (de Lange et al., 2003). Under capacitance measurements, the very rapid capacitance drop of 20-500 fF within 30-500 ms was sometimes observed at the calyx of Held ( $\mathrm{Wu}$ and $\mathrm{Wu}, 2007$ ). So far, the 
underlying mechanisms of bulk endocytosis are unclear. Since bulk endocytosis is only induced under elevated $\left[\mathrm{Ca}^{2+}\right] \mathrm{i}$, calcium-dependent protein phosphatase calcineurin was proposed as a calcium sensor for bulk endocytosis (Cousin, 2009). Calcineurin has an affinity for $\mathrm{Ca}^{2+}$ in the low micromolar range; therefore, it is suitable for detecting an increase in the bulk $\mathrm{Ca}^{2+}$ following massive stimulation of the presynaptic terminal (Clayton and Cousin, 2008; Kumashiro et al., 2005). The function of calcineurin in bulk endocytosis remains to be elucidated.

Presynaptic $\mathrm{Ca}^{2+}$ influx through voltage-gated $\mathrm{Ca}^{2+}$ channels triggers exocytosis of synaptic vesicles. However, the role of $\mathrm{Ca}^{2+}$ in endocytosis still remains unclear. In retinal bipolar cells, $\mathrm{Ca}^{2+}$ may inhibit endocytosis (von Gersdorff and Matthews, 1994); while in other preparations, $\mathrm{Ca}^{2+}$ either accelerates the rate of endocytosis or has no apparent effects ( $\mathrm{Wu}$ et al., 2007). In the calyx of Held synapse, capacitance measurements in combination with $\mathrm{Ca}^{2+}$-uncaging revealed that slow mode of endocytosis depends on local $\left[\mathrm{Ca}^{2+}\right]_{\mathrm{i}}$, at least $10 \mu \mathrm{M}\left[\mathrm{Ca}^{2+}\right]_{\mathrm{i}}$ is required for endocytosis (Hosoi et al., 2009). It is still not clear whether $\mathrm{Ca}^{2+}$ initiates endocytotic events and/or $\mathrm{Ca}^{2+}$ has only a modulatory role in endocytosis.

Calmodulin is a ubiquitously expressed protein, which has four $\mathrm{Ca}^{2+}$-binding sites. Two sites have higher affinity for $\mathrm{Ca}^{2+}$, while other two have lower affinity (Jurado et al., 1999). They work cooperatively: When one site is occupied by $\mathrm{Ca}^{2+}$, the affinity of the other sites will be increased (Burgoyne and Clague, 2003). Binding of $\mathrm{Ca}^{2+}$ to calmodulin leads to a conformational change of calmodulin, exposing its hydrophobic residues which promote the interactions of $\mathrm{Ca} / \mathrm{calmodulin}$ complex to numerous target molecules. For example, calmodulin-dependent protein kinases mediate protein phosphorylation. Calmodulin is involved in many cellular processes, such as LTP, LTD, de novo protein synthesis, gene transcription, neuronal development, synaptic plasticity which might have impacts on behaviors (Wayman et al., 2008). Signaling cascades seem rather complex, and the formation of $\mathrm{Ca} /$ calmodulin complex largely depends on its $\mathrm{Ca}$ affinities, subcellular localization of calmodulin, and the $\mathrm{Ca}^{2+}$ dynamics (Burgoyne et al., 2004). Usually $\mathrm{Ca}$ signals are very transient, and how $\mathrm{Ca} / \mathrm{calmodulin}$ carries out its functions for longer time period depends on the life time of the effetors being activated. For example, autophosphorylation in Thr286 in CaMK by interaction of $\mathrm{CaMK}$ and $\mathrm{Ca} /$ calmodulin 
complex results in prolonged CaMK activity until Thr286 is dephosphorylated (Colbran, 2004). At the calyx of Held, calmodulin is involved in synaptic vesicle replenishment. Pharmacologically block of calmodulin activity slowed down the recovery of fastreleasing vesicles after $50 \mathrm{~ms}$ depleting pulse (Sakaba and Neher, 2001).

Although $\mathrm{Wu}$ et al. suggested that $\mathrm{Ca}^{2+}$ and calmodulin initiate all forms of endocytosis at the calyx of Held, a recent paper from Takahashi's group suggested that $\mathrm{Ca}^{2+}$-dependent endocytosis undergoes developmental change: Nanodomain $\mathrm{Ca}^{2+}$ triggers endocytosis, but in younger calyces (P7 to P9), the bulk $\mathrm{Ca}^{2+}$ that accumulates outside of nanodomain modulates fast and slow endocytosis, and $\mathrm{Ca} /$ calmodulin-calcineurin signaling is involved in the downstream of bulk $\mathrm{Ca}^{2+}$ (Yamashita et al, 2010). On the other hand, the $\mathrm{Ca}^{2+}$ sensor for nanodomain $\mathrm{Ca}^{2+}$ is not clear, and calmodulin is not involved in mature calyces. It seems unlikely that calmodulin is the $\mathrm{Ca}^{2+}$ sensor for all forms of endocytosis, but it remains to be investigated how calmodulin modulates endocytosis. The uncertainty may partially arise from the diversity of the endocytotic kinetics within a cell as well as among different preparations (Smith et al., 2008; Wu, 2004).

Different forms of endocytosis may underlie molecularly different mechanisms. Calyx of Held synapse has all three forms of endocytosis mentioned above, depending on the strengths of stimuli. Unfortunately, the techniques to measure endocytosis with high temporal resolution, such as capacitance measurements and high resolution microscopy are only applicable to special types of synapse such as hippocampal cultures and a large terminal such as the calyx of Held. Therefore, characterizing the mechanisms of endocytosis at the calyx of Held will help us to understand general principles about membrane retrieval in the CNS. Specifically, I focus on the modulation by second messengers, because this has not been studied extensively so far. In this study, I will present the data that calmodulin modulates the endocytotic capacity but does not initiate endocytosis. In addition, it will be shown that cAMP/PKA is the downstream target of calmodulin and modulates the endocytotic capacity. 


\subsection{The aim of the present study}

Short-term plasticity can be affected by many factors; second messengers contribute a pivotal role in the modulation of the synaptic effiency. Among those second messengers, $\mathrm{Ca}^{2+}$ is well established as a trigger for exocytosis and endocytosis at the presynaptic terminal. However, the roles of other second messengers remain unclear. This is because of the lack of techniques available to examine the role of second messengers quantitatively at the presynaptic terminal. In this study, I foused on the role of cAMP, one of the most important second messengers in the cellular signaling. Particularly, cAMP is postulated to be involved in the modulation by hormones as well as short- and long-term presynaptic plasticity. I took advantage of the calyx of Held synapse, where double patch-clamp recordings could be performed, and examined how cAMP modulates the exocytotic and endocytotic processes. It will be shown that cAMP activates wo different signaling molecules, Epac and PKA, which in turn modulate exocytosis and endocytosis, respectively. 


\section{Materials and Methods}

\subsection{Slice preparation}

The preparation of acute thin brain slices, which allows electrophysiological recordings from the neurons in the mammalian CNS was followed by the general guideline provided by (Edwards et al., 1989). More specifically, brain stem slices which contain medial nucleus of the trapezoid body (MNTB) were prepared as described by Forsythe and Barnes-Davies (Forsythe and Barnes-Davies, 1993) and Borst et al. (Borst et al., 1995).

Postnatal days 8 to 11 Wistar rats were decapitated with a moderate scissors (according to the guidelines of German laws on animal protection) and the head was immediately transferred into a dish which contains $0.1 \mathrm{mM} \mathrm{Ca}^{2+}$ ice cold artificial cerebrospinal fluid (described below in solution preparation). The purpose of using this cold, low $\mathrm{Ca}^{2+}$ solution is to lower the temperature of the tissue, thus lowering the metabolism and preventing anoxia of the neurons. In order to exposure the skull, the skin on the dorsal part of the head was cut in the middle line. The skull was opened with a fine scissors. Two transverse cuts were made in the brain in order to take out the brainstem, one close to the mouth, another one close to Bregma point in the cerebrum part (for the second cut, see the blank line in Fig. 2-1). The brainstem together with cerebellum was carefully removed from the skull. Usually there are several tiny blood vessels stuck to the ventral part of the brainstem and fine forceps were used to remove these vessels. The reason for removal is that during slicing these vessels are tended to drag the slice, such that cells which close to the edge of the slice might be destroyed by the tension. Cyanoacrylate glue was used to fix the brainstem to the slicing chamber, which also contained ice cold artificial cerebrospinal fluid. The ventral part of the brainstem should directly face the slicing blade.

$200 \mu \mathrm{m}$ thick transverse brain slices were made using a vibratome slicer (Integraslice 7550 MM, Campden Instruments, Leicester, UK or Leica VT 1200S) with the horizontal vibration set at $70 \mathrm{~Hz}$ and advancing $0.10-0.18 \mathrm{~mm} / \mathrm{s}$. The seventh cranial nerve ( 7 th nerve) was used as a landmark for the start of the MNTB region. Usually the 
slice which contains the 7th nerve had very few MNTB principle cells, and 5 to 6 slices after the 7 th nerve were collected and were transfered slices into an incubation chamber, which contained normal $2 \mathrm{mM} \mathrm{Ca}{ }^{2+}$ extracellular solution continuously bubbled with $95 \% \mathrm{O}_{2}$ and $5 \% \mathrm{CO}_{2}$ at $36^{\circ} \mathrm{C}$ (at least $30 \mathrm{~min}$ to $1 \mathrm{~h}$ before the experiment).

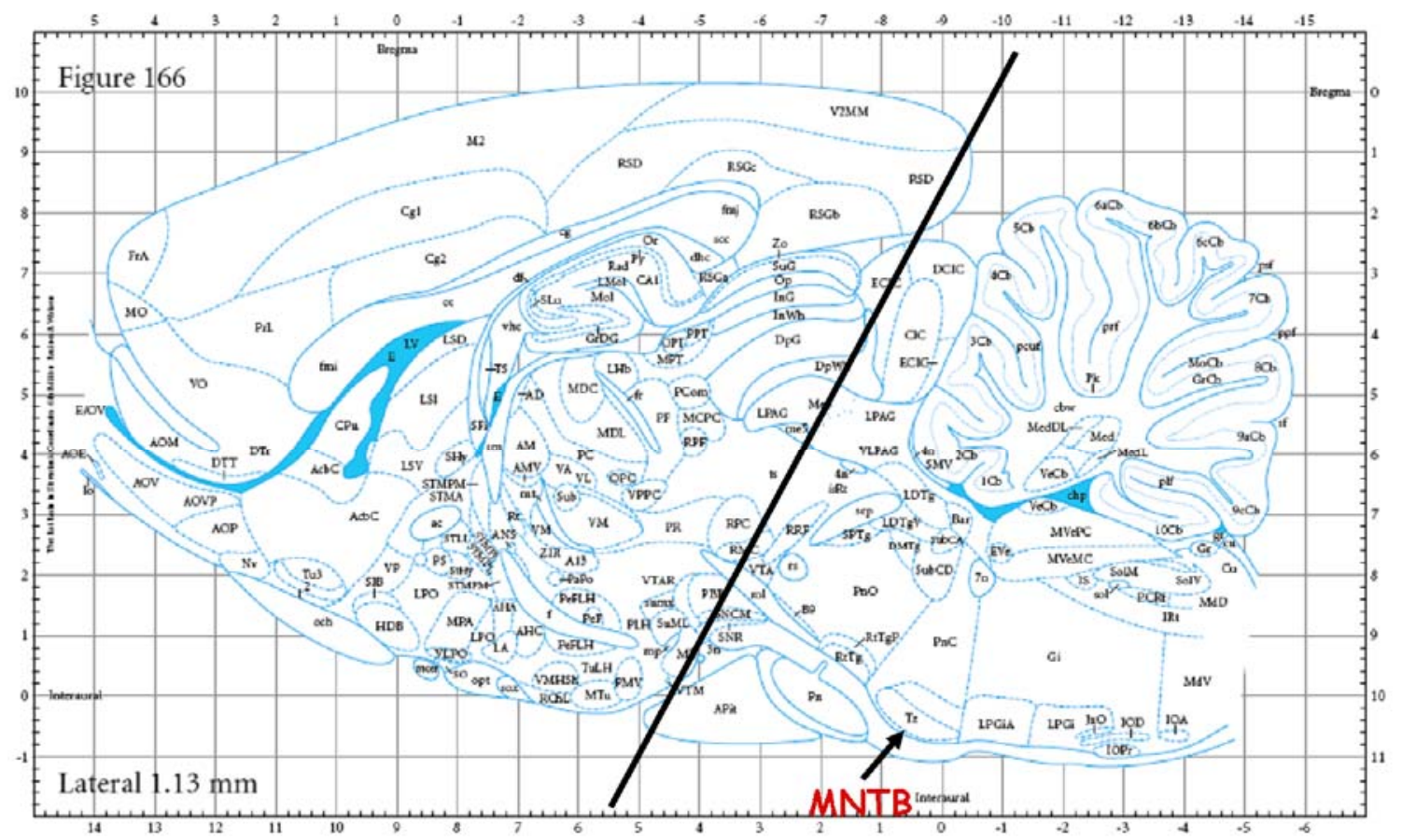

\section{Figure 2-1: Lateral view of adult rat brain.}

The black line shows where the cut was performed to separate the cerebrum and brainstem from forebrain. The brainstem together with cerebellum was fixed to a slicing disk with glue. MNTB region was indicated with an arrow in the picture. During slicing, the seventh cranial nerve was used as a landmark for the starting point of MNTB. The first slice was taken after disappearnce of the seventh cranial nerve. Up to six slices were collected which may contain MNTB region.

\subsection{Solutions}

\subsubsection{Extracellular solutions}


In this section all the drugs I used for my $\mathrm{PhD}$ thesis will be listed. The composition of slicing and normal external solution was the same, except the concentration of $\mathrm{Ca}^{2+}$ and $\mathrm{Mg}^{2+}$ were different. All the experiments were performed at room temperature.

1) Artificial cerebrospinal fluid used for slicing (mM): $125 \mathrm{NaCl}, 2.5 \mathrm{KCl}, 0.1 \mathrm{CaCl}_{2}$, $3 \mathrm{MgCl}_{2}, 25$ glucose, $25 \mathrm{NaHCO}_{3}, 1.25 \mathrm{Na}_{2} \mathrm{PO}_{4}, 0.4$ L-ascorbic acid, 3 myo-inositol and 2 Na-Pyruvate. $\mathrm{pH} 7.3-7.4,300 \mathrm{mOsm}$. The slicing solution was kept in $-80{ }^{\circ} \mathrm{C}$ for $20-$ 30 minutes before slicing in order to lower the temperature until the solution became icewater mixture. Then the solution was bubbled with $95 \% \mathrm{O}_{2}$ and $5 \% \mathrm{CO}_{2}$ before and during slicing.

2) Normal external solution (mM): $125 \mathrm{NaCl}, 2.5 \mathrm{KCl}, 2 \mathrm{CaCl}_{2}, 1 \mathrm{MgCl}_{2}, 25$ glucose, $25 \mathrm{NaHCO}_{3}, 1.25 \mathrm{Na}_{2} \mathrm{PO}_{4}, 0.4$ L-ascorbic acid, 3 myo-inositol and 2 Na-Pyruvate. $\mathrm{pH}$ 7.3-7.4, 300 mOsm, bubbled continuously with $95 \% \mathrm{O}_{2}$ and $5 \% \mathrm{CO}_{2}$. Depending on the types of the experiments, different kinds of pharmacological compounds were added.

3) Pharmacological drugs used during the patch-clamp recording: During the recording, $1 \mathrm{mM}$ kynurenic acid (Kyn), $10 \mathrm{mM}$ tetraethylammonium chloride (TEA-Cl), $1 \mu \mathrm{M}$ tetrodotoxin (TTX), 50 $\mu \mathrm{M} \mathrm{D}(-)$-2-amino-5-phosphonopentanoic acid (D-AP5), $100 \mu \mathrm{M}$ cyclothiazide (CTZ) were added to isolate pre-synaptic $\mathrm{Ca}^{2+}$ currents and postsynpatic AMPA receptor-mediated EPSCs. The action of drugs used in these type of experiments are listed below:

Kyn: a low-affinity competitive antagonist of glutamate receptors

TTX; sodium channel blocker

TEA-Cl: potassium channel blocker

D-AP5: competitive NMDA receptor antagonist

CTZ: an inhibitor of glutamate receptor desensitization

At the calyx of held synapse, the mEPSC amplitude decreases strongly during strong stimulation due to postsynaptic receptor desensitization. Constant mEPSC sizes have to be assumted for the deconvolution method, which was used for quantitative analysis of the kinetics of vesicle release. Application of Kyn and CTZ prevent postsynaptic receptor desensitization. In the presence of CTZ, the EPSCs decay much more slowly than without CTZ, and the residual currents due to pooling of glutamate in the synaptic cleft deveop during the stimulation. The residual currents are not mediated by the direct 
release events, and have to be subtracted from the total current with the model fitting (Neher and Sakaba, 2001). $1 \mathrm{mM}$ Kyn, which reduce the EPSC amplitudes to $50 \%$, removed the possible postsynaptic receptor saturation during intense stimulation, and also prevented voltage-clamp problems. Constant mEPSC sizes in the presence of CTZ and Kyn have been verified by fluctuation analysis (Neher and Sakaba, 2001).

Since Kyn can absorb UV light and therefore interfere with the Ca imaging, $2 \mathrm{mM}$ $\gamma$-D-glutamylglycine ( $\gamma$-DGG), which also block postsynaptic receptor saturation, was used instead of Kyn during flash photolysis experiments.

For the miniature EPSC recording, CTZ and Kyn were omitted. In this type of experiment, the vesicle release rates were quite low, so desensitization and saturation were not supposed to be serious. $10 \mu \mathrm{M}$ bicuculline $\left(\mathrm{GABA}_{\mathrm{A}}\right.$ receptors blocker) and 10 $\mu \mathrm{M}$ strychnine (glycine receptors antagonist) were added to block potential small inhibitory inputs.

For capacitance measurements, $1 \mu \mathrm{M}$ TTX and $10 \mathrm{mM}$ TEA-Cl were included in the normal external solution. In some experiments, $50 \mu \mathrm{M}$ MDL-12,330A (a cell-permeable irreversible inhibitor of adenylate cyclase), $100 \mu \mathrm{M} 8$-(Chlorophenylthio)-2'-O-methylcAMP (8-pCPT-2'-O-Me-cAMP, a potent, specific and membrane-permeant activator of the Epac) were additionaly included in the extermal solution.

CTZ, $\gamma$-DGG, Kyn, D-AP5 bicuculline and strychnine were obtained from Tocris. Froskolin, 3-isobutyl-1-methylxanthine (IBMX) and MDL-12 330A were obtained from Calbiochem. 8-pCPT-2'-O-Me-cAMP was obtained from BioLog. Other drugs were obtained from Sigma. CTZ was dissolved in DMSO, for a final concentration in the extracellular solution of $0.1 \%$.

\subsubsection{Intracellular solution}

In all of the experiments, cesium-gluconate based intracellular solution was used both for pre- and postsynaptic recordings. Different concentrations of EGTA were added to the pre- and postsynaptic pipette solution. Different types of peptides and antibodies were included in the pre-pipette solution depending on the purpose of the experiments.

Pre-synaptic internal solution (mM): 135 Cs-gluconate, 20 TEA-Cl, 10 HEPES, 5 $\mathrm{Na}_{2}$-phosphocreatine, 4 MgATP, 0.3 GTP, 0.2 mM EGTA, pH adjust to 7.2. $100 \mathrm{mM}$ Cs- 
gluconate stock was used to adjust the osmolarity to 330 mOsm. For capacitance measurement, $0.5 \mathrm{mM}$ EGTA was added in most of the experiments, except indicated in the results.

Postsynaptic internal solution (mM): 135 Cs-gluconate, 20 TEA-Cl, 10 HEPES, 5 $\mathrm{Na}_{2}$-phosphocreatine, $4 \mathrm{MgATP}, 0.3 \mathrm{GTP}$, and 5 EGTA, pH adjust to 7.2.

Presynaptic internal solution for flash photolysis (mM): 120 Cs-gluconate, 20 TEA-Cl, 20 Hepes, $0.5 \mathrm{MgCl}_{2}$, $5 \mathrm{Na}_{2} \mathrm{ATP}, 0.5 \mathrm{NaGTP}, 2 \mathrm{DM}$-nitrophen, and 0.2 fura$2 \mathrm{FF}, \mathrm{pH}$ adjust to $7.2,330$ mosm. For weak flash experiments, a high affnity $\mathrm{Ca}^{2+}$ indicator 0.1 fura-4F was used instead of fura-2FF, and $0.85-1.7 \mathrm{CaCl}_{2}$ was included in the solution in order to clamp the basal, pre-flash $\left[\mathrm{Ca}^{2+}\right]_{\mathrm{i}}$ below $50 \mathrm{nM}$. Osmolarity was adjusted to 330 .

Presynptic solution for the mEPSC recording $(\mathrm{mM})$ : For this type of experiment, 4 times internal solution was prepared: 400 Cs-gluconate, 80 Hepes, 16 Mg-ATP, 1.2 $\mathrm{Na}_{2} \mathrm{ATP}$, and 80 TEA-Cl. In order to clamp $\left[\mathrm{Ca}^{2+}\right]_{\mathrm{i}}$ to the concentration below $500 \mathrm{nM}$, $20 \mathrm{mM}$ EGTA together with different concentrations of $\mathrm{Ca}^{2+}$ was added to the 4 times solution, in addition to $\mathrm{Ca}^{2+}$ indicator fura-4F The final solution was diluted with doubledistilled water, and the osomlarity was adjusted to 330 by adding Cs-gluconate stock.

\subsection{Double patch-clamp recordings}

For electrophysiological recording, a slice was placed on a custom-built recording chamber and was covered with a platinum U-shaped grid with parallel Nylon filaments. After fixing the recording chamber on the platform of the upright microscope BX51WI (Olympus, Tokyo, Japan) equipped with a 10 X 60 objective, the slice was continuously perfused with the extracellular solution $95 \% \mathrm{O}_{2}$ and $5 \% \mathrm{CO}_{2}$. The extracellular solution was prefused through a custom-built gravity driven perfusion system. There were several syringe tubes conncted to the perfusion system, so that different kinds of perfusion solutions could be applied depending on the aim of the experiments. The flow rate of the solution was adjustable by changing the open extent of the connecting tube. All the recordings were performed at room temperature $\left(22-25^{\circ} \mathrm{C}\right)$ with an EPC-10/2 patchclamp amplifier (HEKA, Lambrecht, Germany). Electrical responses were sampled with 
Pulse or Patch-master software (HEKA, Germany).

Patch pipettes were pulled from borosilicate glass filaments $(2 \mathrm{~mm}$ diameter, 0.3 $\mathrm{mm}$ wall thickness from Hilgenberg, Malsfeld, Germany) using a vertical temperaturecontrolled two-step pipette puller (PIP 5 from HEKA, Lambrecht/Pfalz, Germany). The presynaptic terminal has a finger-like structure with a diameter around $1 \mu \mathrm{m}$, while the post- principle neuron has a much bigger size with the diameter between $15-25 \mu \mathrm{m}$. Presynaptic patch pipettes have a longer tip and a small open diameter with a pipette resistance between $5-6 \mathrm{M} \Omega$. The postsynaptic patch pipettes have a relatively short tip and a big open diameter with a resistance between $3-5 \mathrm{M} \Omega$.

The pre- and postsynaptic components were simultaneously whole-cell voltage clamped at $-80 \mathrm{mV}$. The fast and slow capacitances, refleting the pipette and the cell capacitance, respetively, were automatically compensated using a built-in compensation circuitry. The series resistance was around 8-25 M $\Omega$ for the presynaptic recording and 4$10 \mathrm{M} \Omega$ for postsynaptic recording. The presynaptic series resistance was electronically compensated by the amplifier by $30-90 \%$. The postsynaptic series resistance was compensated by $30-70 \%$. The uncompensated resistance was below $8 \mathrm{M} \Omega$ for pre- and 3 $\mathrm{M} \Omega$ for post-synaptic recording. The remaining resistance was further compensated offline by Igor macros. Data were sampled at $20-50 \mathrm{kHz}$ with a low-pass filter at corner frequency of $6 \mathrm{kHz}$.

\section{4 $\mathrm{Ca}^{2+}$-uncaging and $\left[\mathrm{Ca}^{2+}\right]_{\mathrm{i}}$ imaging}

The $\mathrm{Ca}^{2+}$-uncaging technique in combination with quantitative $\left[\mathrm{Ca}^{2+}\right]_{\mathrm{i}}$ imaging was successfully applied to the calyx of Held synapse in the brainstem slice by Schneggenburger and Neher (Schneggenburger and Neher, 2000). In this part, the technique is described in detail.

$\mathrm{Ca}^{2+}$ was uncaged from the $\mathrm{Ca}^{2+}$-loaded DM-nitrpphen (DMN) using a flash lamp. DMN has a high affinity for $\mathrm{Ca}^{2+}\left(\mathrm{K}_{\mathrm{D}}=5 \mathrm{nM}\right)$, Because of the high affinity, most $\mathrm{Ca}^{2+}$ is bound to DMN, and low concentration of free $\mathrm{Ca}^{2+}(\sim 100 \mathrm{nM})$ is maintained. Upon a UV flash, the affinity for $\mathrm{Ca}^{2+}$ is largely decreased $\left(\mathrm{K}_{\mathrm{D}}=3 \mathrm{mM}\right)$. Therefore $\mathrm{DMN}$ is an ideal candidate for controlling the intracellular $\mathrm{Ca}^{2+}$ level. The purity of DMN is different among batches. Titration with $\mathrm{CaCl}_{2}$ was made to examine the purity of DMN. Usually a 
purity of $70-80 \%$ was obtained, and this value was taken into account when making the final intracellular solution. Ratiometric $\mathrm{Ca}^{2+}$ indicators were used to determine the exact $\left[\mathrm{Ca}^{2+}\right]_{\mathrm{i}}$ in our experiments. The $350 / 380 \mathrm{~nm}$ excitation ratio for fura-2FF was used, supplied by a monohromator (TILL, Photonics, Gräfelfing, Germany) and the emitted fluoresnece was collected at $510 \mathrm{~nm}$, the maximal emission wavelength.

The equation for calculating $\left[\mathrm{Ca}^{2+}\right]_{\mathrm{i}}$ is as follow:

$$
\left[\mathrm{Ca}^{2+}\right]=K_{\mathrm{eff}} \frac{R-R_{\min }}{R_{\max }-R}
$$

Here, all the calibration constants $\mathrm{K}_{\text {eff }}, \mathrm{R}_{\min }$ and $\mathrm{R}_{\max }$ were determined experimentally. The ratio $\mathrm{R}$ was measured before and after photolysis. Calibration of the dyes were carried out in vitro, as follows: $\mathrm{R}_{\min }$ was determined separately in vitro using the solution with free $\left[\mathrm{Ca}^{2+}\right]_{i}$ of 0 , while $\mathrm{R}_{\max }$ was obtained with the solution of free $\left[\mathrm{Ca}^{2+}\right]_{\mathrm{i}}$ of $10 \mathrm{mM}$. An intermediate $\left[\mathrm{Ca}^{2+}\right]_{\mathrm{i}}$ of $10 \mu \mathrm{M}$ was used to calculate $\mathrm{K}_{\text {eff. }}$ The $\mathrm{Kd}$ of fura-2FF is $\sim 10 \mu \mathrm{M}$, and therefore, one can use this dye to measure $\left[\mathrm{Ca}^{2+}\right]_{\mathrm{i}}$ between 1 $\mu \mathrm{M}$ and $50 \mu \mathrm{M}$ relatively accurately. For weak flash and Ca-loading experiments, fura- $4 \mathrm{~F}$ was used for accurate measuring the $\left[\mathrm{Ca}^{2+}\right]_{\text {i }}$, below $1 \mu \mathrm{M}$, which has a $\mathrm{Kd}$ around $1 \mu \mathrm{M}$. All the parameters for fura- $2 F F$ were: $K_{\text {eff }}=92.5537, R_{\min }=0.4297$, and $R_{\max }=5.2691$. For fura-4F, $\mathrm{K}_{\text {eff }}=17.9254, \mathrm{R}_{\min }=0.444$ and $\mathrm{R}_{\max }=11,411$ were obtained. These parameters were used in all Ca-imaging experiments. All the solutions were used for calibration and flash are listed in Table 2-1and 2-2.

Table 2-1: Four-fold basic solution, which was diluted when making final solution for calibration and recordings in the flash experiments.

\begin{tabular}{|l|l|}
\hline compounds & concentration $(\mathrm{mM})$ \\
\hline Ca-gluconate & 400 \\
\hline HEPES & 80 \\
\hline Na2-ATP & 20 \\
\hline Na2-GTP & 1.2 \\
\hline MgCl26H2O & 2 \\
\hline
\end{tabular}




\begin{tabular}{|l|l|}
\hline TEA-Cl & 80 \\
\hline
\end{tabular}

Fluorescent images were obtained with in interline-transfer 640 X 480 pixel CCD, controlled by the software Tillvision. Images were binned by $8 \times 16$. The flash light intensity was attenuated by neutral density filters to produce the $\left[\mathrm{Ca}^{2+}\right]_{i}$ steps of different amplitudes. Images were analyzed off-line with Tillvision, and the resulting time series of fluorescence intensities of several pixels and the background fluorescence of a small area next to the calyx were transferred to Igor Pro 5.05A.s for additional analysis.

Table 2-2: Solutions for calibration and flash experiments. The final volume was adjusted by double-distilled water.

\begin{tabular}{|l|l|}
\hline compounds & final concentration (mM) \\
\hline $\mathbf{R}_{\min }$ & \\
\hline 4-fold solution & \\
\hline Fura-2FF & 0.05 \\
\hline DMN & 1 \\
\hline EGTA & 10 \\
\hline Ca-gluconate & 25 \\
\hline $\mathbf{R}_{\max }$ & \\
\hline 4-fold solution & \\
\hline Fura-2FF & 0.05 \\
\hline DMN & 1 \\
\hline CaCl2 & 10 \\
\hline Ca-gluconate & 25 \\
\hline $\mathbf{R}_{10 \mu \mathrm{M}}$ & \\
\hline 4-fold solution & \\
\hline Fura-2FF & 0.05 \\
\hline DMN & 1 \\
\hline DPTA-CaCl2 & $10-2.5$ \\
\hline Cs-gluconate & 25 \\
\hline Flash photolysis experiments & \\
\hline 4 fold solution & \\
\hline Fura-2FF & 0.2 \\
\hline
\end{tabular}




\begin{tabular}{|l|l|}
\hline $\mathrm{DMN}$ & 2 \\
\hline $\mathrm{CaCl} 2$ & 1.7 \\
\hline Ca-gluconate & $25-30$ \\
\hline
\end{tabular}

\subsection{The deconvolution method}

In order to obtain the transmitter release rates, one can count the number of mEPSCs directly, but this method can be only applied when the release rates are very low and individual events can be resolved. With the increase of the vesicle fusion rates, individual vesicles are merged with each other, leading to the compound EPSCs. Under this condition, deconvolution of the EPSCs with the miniature EPSC waveform was used to calculate the release rates. This method assumes that postsynaptic currents are a linear summation of individual mEPSCs, which is experimentally verified at the neuromuscular junction (Hartzell et al., 1975; Magleby and Pallotta, 1981). However, the simple decovolution method cannot be directly applied to the calyx of Held. The calyx of Held synpase has around 550 active zones, each of which contains 5-6 releasable synaptic vesicles. Under strong stimulation, transmitter release tends to be pooled and cleared very slowly from the calyx of Held synapse. The delayed clearance of transmitter from the synaptic cleft induces the residual current. In addition, saturation and desensitization of the postsynaptic AMPA receptors reduce the $\mathrm{mEPSC}$ amplitudes after stimulation. The constant mEPSC size is an important assumption for the deconvoleution method. Neher and Sakaba (2001) developed a simple model, which could estimate the contribution of residual current due to the delayed clearance of glutamate in the synaptic cleft. They incorporated this model to the deconvolution algorithm. By combining deconvolution with fluctuation analysis, it has been shown that this method was valid in the presence of CTZ and kyn, which blocked desensitization and possible saturation of the postsynaptic AMPA receptors, respectively. Quantal release rates in this study were estimated by this adapted deconvolution algorithm. Cumulative release was obtained by integrating the quantal release rates over time (Neher and Sakaba, 2001a, b). After synaptic vesicle pool depletion, new synaptic vesicles were recruited to the synaptic vesicle pool. For 
estimating the size of release-ready vesicle pool, the rates of synaptic vesicle replsihment rates were subtracted from the cumulative release, which were estimated at 50-100 ms after onset of the stimulation.

\subsection{Capacitance measurement}

There are several ways to monitor exocytosis and endocytosis in high temporal resolution. In addition to recording the postsynaptic currents (potentials), amperometric technique has been used to analyze the kinetics of vesicle fusion, but this technique can only be limited to the cells secreting transmitters that can be oxidized. Imaging techniques are also quite often used to study endocytosis, but with somewhat limited time resolution. The time-resolved membrane capacitance measurement is another tool used to monitor the exocytosis and endocytosis in many cells. This technique uses the sinusoidal voltage stimuli based on the idea that target cell is electrically equivalent to a single compartment composed of a membrane capacitor and a membrane resistor. During exocytosis, synaptic vesicles are fused with plasma membrane, leading to an increase of the cell surface area; then, an increase of membrane capacitance is recorded. Exocytosis is followed by endocytosis, and the membrane is pinched off from plasma membrane. This process is also monitored by a capacitance decrease. Therefore, capacitance measurements can be applied to monitor both exocytosis and endocytosis with a resolution of milliseconds range. Disadvantages of this technique include the fact that only the net change of the surface area is reported. Endocytosis might be masked by ongoing exocytisis during the stimulation. However, in most of the cases, exocytosis happens in milliseconds range and endocytosis happens in tens of seconds range, so both are temporally separated

In our experiments, the presynaptic capacitance was measured with an EPC10/2 amplifier in sine + DC configuration (Lindau and Neher, 1988). A sine wave $(30 \mathrm{mV}$ in amplitude, $1000 \mathrm{~Hz}$ ) was superimposed on a holding potential of $-80 \mathrm{mV}$. The peak-topeak voltage was less than $60 \mathrm{mV}$ to avoid activation of $\mathrm{Ca}^{2+}$ channels. The recording pipettes were coated with sylgard to reduce pipette capacitance, and the bath solution level was kept very low to reduce the capacitance. Capacitance changes were induced by 
step depolarizaions or trains of AP-like stimuli. Measurements were accepted for further analysis if the presynaptic series resistance was below $20 \mathrm{M} \Omega$. Otherwise, the data were discarded, because the capcitance trace became unstable upon a subtle change in the series resistatnce.

\subsection{Data analysis}

Patcher's Power Tools (PPT) was used to transfer electrophysilogical data to Igor Pro 5.05A for further analysis. Average data are presented as mean \pm standard error of mean. 


\section{Results}

\subsection{CAMP modulates the intracellular $\mathrm{Ca}^{2+}$ sensitivity of the fast- releasing synaptic vesicles at the calyx of Held synapse}

cAMP is one of the most important second messengers involved in essential cellular signaling pathways. At the calyx of Held synapse, cAMP modulates synaptic vesicle release by increasing the vesicle release probability and/or the number of releasable vesicles, through Epac-mediated pathway (Kaneko and Takahashi, 2004; Sakaba and Neher, 2001b). In the first part of my study, voltage-clamp recordings, $\mathrm{Ca}^{2+}$-imaging and $\mathrm{Ca}^{2+}$-uncaging were combined to address how cAMP modulates synaptic vesicle fusion quantitatively.

\subsubsection{Forskolin increases the synaptic vesicle release at the calyx of Held synapse}

Forskolin, an adenylyl cyclase activator, can potentiate EPSCs evoked by a step depolarization of the presynaptic terminal or by fiber stimulation (Kaneko and Takahashi, 2004; Sakaba and Neher, 2001b). However how cAMP exactly affects the release probability and the size of releasable vesicle pool under more physiological conditions, are still not clear. In order to address this question, double patch-clamp whole-cell recordings of the pre- and postsynaptic compartments of the calyx of Held synapse were performed (slices were prepared from P9 to P11 rats). The EGTA concentration in the presynaptic patch pipette solution was $0.2 \mathrm{mM}$ in order to mimic the physiological intracellular $\mathrm{Ca}^{2+}$ buffering system (Borst and Sakmann, 1996; Müller et al., 2007). Rundown of the EPSCs due to presynaptic whole-cell dialysis tends to show up when the recording lasts longer than 10-15 min although this differs among cells. To observe the cAMP-induced potentiation of transmitter release more rapidly, IBMX $(100 \mu \mathrm{M})$, an inhibitor of phosphodiesterase (which hydrolyzes cAMP or cGMP), was added to the extracellular solution. At the calyx of Held synapse, IBMX only accelerates the forskolininduced potentiation of EPSCs, without changing the amplitude of the potentiation 
(Sakaba and Neher, 2001b). Presynaptic $\mathrm{Ca}^{2+}$ influx was evoked by a 20 pulse, $100-\mathrm{Hz}$ train of AP-like stimuli (depolarization to $+40 \mathrm{mV}$ for 0.4 to $1 \mathrm{~ms}$, and then ramp to -80 $\mathrm{mV}$ for $0.4-1 \mathrm{~ms}$ ), followed by a step-depolarizing pulse (depolarization to $70 \mathrm{mV}$ for 2 ms followed by repolarization to $0 \mathrm{mV}$ for $50 \mathrm{~ms}$ ) to deplete the remaining vesicle pool (see Fig. 3-1, upper panel "Vpre" for the stimulation protocol). The duration of each APlike stimulus was adjusted to achieve a relatively small amplitude of the first EPSC, so that the large potentiation effect could be observed. During the train of AP like stimuli, the EPSC initially showed facilitation (only the EPSC during the second AP), after which depression became dominant. The depression is most likely caused by rapid depletion of the releasable vesicle pool (Schneggenburger et al., 1999; Wu and Borst, 1999).

Extracellular application of forskolin $(50 \mu \mathrm{M})$ and IBMX induced a marked potentiation of the EPSCs (Fig. 3-1, EPSC) without changing the amplitude of presynaptic $\mathrm{Ca}^{2+}$ currents or $\mathrm{Ca}^{2+}$ charge, significantly (Fig. 3-1 $\mathrm{I}_{\mathrm{Ca}}$, Fig. 3-2 for average value). The release rates were calculated from the EPSCs by the deconvolution method (see Methods) (Neher and Sakaba, 2001a, b), assuming that forskolin + IBMX did not modulate the miniature EPSC (mEPSC) amplitudes, as verified in previous studies (Sakaba and Neher, 2001b). The peak release rate during the first AP was 930 quanta ms ${ }^{-}$ ${ }^{1}$ and the cumulative release was 10,500 quanta under control conditions (at the end of the $50 \mathrm{~ms}$ pulse) (Fig. 3-1). After application of forskolin + IBMX, the peak release rate of the first EPSC was increased to 2360 quanta $\mathrm{ms}^{-1}$, and the cumulative release was increased to 13,390 quanta. A subsequent step-depolarizing pulse after the AP-like stimuli evoked only relatively slow release, indicating that the fast-releasing synaptic vesicles responsible for AP-triggered release had been mostly depleted during AP-like stimuli (Sakaba, 2006).

On average, the amount of presynaptic $\mathrm{Ca}^{2+}$ influx was identical between control and forskolin-treated conditions $(1.00 \pm 0.04$-fold, $\mathrm{P}=0.95, \mathrm{n}=9)$ (Fig. 3-2). The amplitude of the first EPSC in an AP train was increased more than 2-fold (2.21 \pm 0.36 fold, $\mathrm{P}<0.01, \mathrm{n}=9$ ). Large potentiation was observed especially during the initial few stimuli in the AP train (usually the first 5 - 6 stimuli). The peak release rate of the first stimulus was also increased 2 -fold $(2.1 \pm 0.32$-fold, $\mathrm{P}<0.01)$. The size of the releasable vesicle pool was calculated from the cumulative release, as described (see Methods). The 
pool size was variable among cell pairs under control conditions (1600 to 13,500 vesicles; mean $=6200 \pm 1680, n=8$ ); this number includes both the fast- and the slowly releasing vesicles at the calyx of Held synapse (Sakaba and Neher, 2001). On average, forskolin increased the total size of releasable vesicles by $20 \%(1.20 \pm 0.04, \mathrm{P}<0.001, \mathrm{n}=9)$ (Fig. $3-2)$.

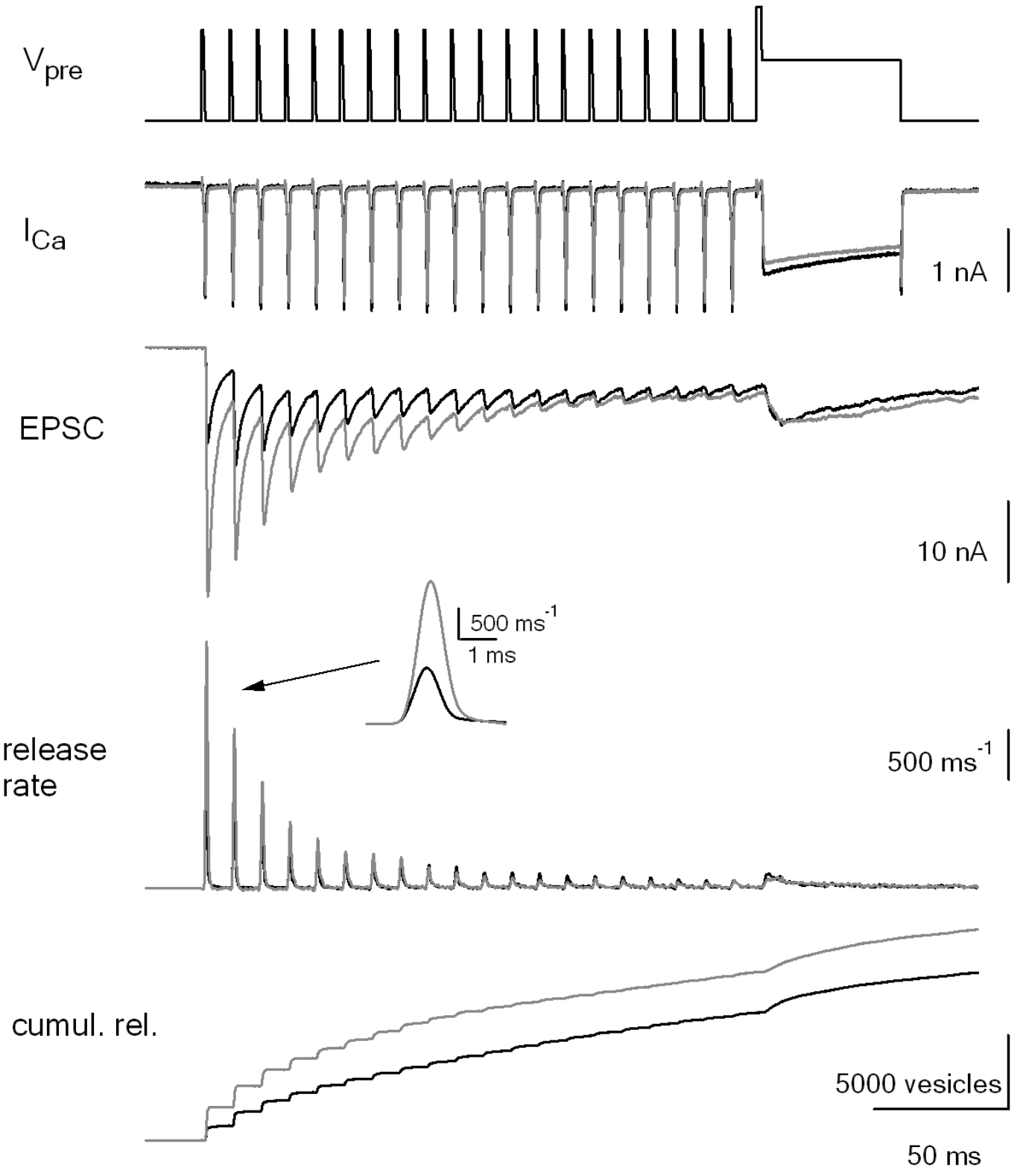

Figure 3-1: Potentiation of EPSCs by forskolin under a train of AP-like stimuli. 
Both pre- and postsynaptic compartments were simultaneously patch-clamped at $-80 \mathrm{mV}$ through two glass pipettes. A $100 \mathrm{~Hz}$ train of 20 AP-like stimuli (depolarization to $+40 \mathrm{mV}$ for 0.4 to $1 \mathrm{~ms}$, then ramped to $-80 \mathrm{mV}$ for $0.8 \mathrm{~ms}$ ) was applied to the calyx of Held terminal through a patch pipette, followed by a step-depolarizing pulse to deplete the remaining pool of releasable vesicles (pre-pulse to $70 \mathrm{mV}$ for $2 \mathrm{~ms}$ followed by a repolarization pulse to $0 \mathrm{mV}$ for $50 \mathrm{~ms}$ ). From top to bottom, the stimulation protocol, presynaptic $\mathrm{Ca}^{2+}$ currents, EPSCs, release rates and the cumulative release are shown. The inset shows the release rates evoked by the first AP-like stimulus both under control and forskolin-treated conditions (black: control; grey: forskolin, applied to all subsequent figures).

The release probability was obtained by dividing the number of vesicles released during each AP by the vesicle pool size. Forskolin increased the release probability of the first six stimuli in a stimulus train, and the potentiation was largest during the first stimulation. On average, the release probability in response to the first stimulus increased $1.84 \pm 0.22$-fold $(\mathrm{P}<0.01, \mathrm{n}=9$ ) (Fig. 3-2). These results suggest that forskolin + IBMX increases both the release probability (by $80 \%$ ) and the number of readily releasable vesicles (by $20 \%$ ) during a train of AP-like stimuli at the calyx of Held synapse. Increase in release probability could be due to a tighter coupling between $\mathrm{Ca}^{2+}$ channels and synaptic vesicles (Wadel et al., 2007) or increased $\mathrm{Ca}^{2+}$ sensitivity of synaptic vesicles for fusion. In the following experiments, flash photolysis of caged $\mathrm{Ca}^{2+}$ was used to directly address how cAMP modulates the $\mathrm{Ca}^{2+}$ sensitivity for vesicle fusion (Lou et al., 2005; Schneggenburger and Neher, 2000).

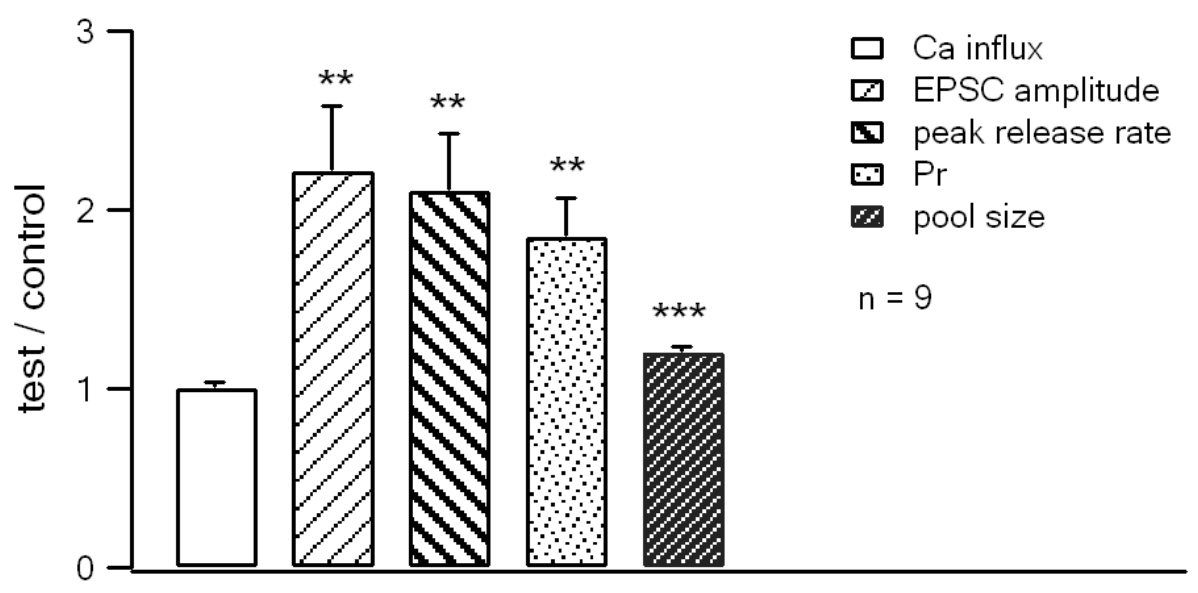


Figure 3-2: Average amount of presynaptic $\mathrm{Ca}^{2+}$ influx, EPSC amplitude, the peak release rate, the release probability during the first AP-like stimulus, and the size of the releasable pool of vesicles.

Presynaptic $\mathrm{Ca}^{2+}$ influx was calculated by integrating the Ca current with time. The readily releasable pool size was calculated from cumulative release by subtracting newly recruited vesicles; see details in material and methods. The release probability during the first AP was divided the released vesicle number during the first AP by the total size of synaptic vesicle pool. The data were collected from 9 pairs, and normalized to the control value in a given cell pair to illustrate the magnitude of the forskolin-induced potentiation (release probability, $\operatorname{Pr} ;{ }^{* *}, P<0.01 ; * * *, P<0.001$ ).

\subsubsection{Forskolin increases transmitter release induced by presynaptic $\mathrm{Ca}^{2+}$ uncaging}

It is well established that synaptic vesicle fusion is $\mathrm{Ca}^{2+}$-dependent, and $\mathrm{Ca}$ sensors are far from being saturated during APs. Change in the intracellular $\mathrm{Ca}^{2+}$ sensitivity can potentially affect the output of the signals, thus any factor which can modulate the the $\mathrm{Ca}^{2+}$ sensitivity is important for synaptic plasticity. In order to study how cAMP affects the $\mathrm{Ca}^{2+}$-dependent synaptic vesicle fusion quantitatively, simultaneous pre- and postsynaptic whole-cell recordings were performed in combination with presynaptic $\mathrm{Ca}^{2+}$ uncaging, which elevates the intracellular $\mathrm{Ca}^{2+}$ concentration uniformly in the terminal. $\left[\mathrm{Ca}^{2+}\right]_{\mathrm{i}}$ was monitored using the $\mathrm{Ca}^{2+}$ indicator dye fura-2FF, and DMN was used as caged component for $\mathrm{Ca}^{2+}$.-uncaging. Application of an $\mathrm{UV}$ flash photolyzes DMN and elevates $\left[\mathrm{Ca}^{2+}\right]_{\mathrm{i}} .60 \mathrm{~ms}$ after flash photolysis of caged $\mathrm{Ca}^{2+}$, a step-depolarizing pulse was applied to deplete the remaining releasable vesicles. Different flash intensities were used to elevate $\left[\mathrm{Ca}^{2+}\right]_{\mathrm{i}}$ to different levels. Fig. 3-3 shows an example of a flash application of intermediate intensity, which elevated $\left[\mathrm{Ca}^{2+}\right]_{\mathrm{i}}$ from basal level to $5.5 \mu \mathrm{M}$ under control conditions and to $4.8 \mu \mathrm{M}$ after the application of forskolin + IBMX. Although the peak $\left[\mathrm{Ca}^{2+}\right]_{\mathrm{i}}$ level was slightly lower after the application of forskolin + IBMX than that under control condition, the peak amplitude of flash-evoked EPSC increased from $2.5 \mathrm{nA}$ to 4.7 $\mathrm{nA}$. The peak release rate increased from 74 vesicles $\mathrm{ms}^{-1}$ to 150 vesicles $\mathrm{ms}^{-1}$. The size of the releasable vesicle pool (at the end of a depleting pulse) also increased by $16 \%$ in this cell pair ( $21 \pm 7 \%$ on average when the post-flash $\left[\mathrm{Ca}^{2+}\right]_{\mathrm{i}}$ was between 3 to $6 \mu \mathrm{M} ; n$ $=5$ ), similar to the result obtained from a train of AP-like stimuli. 
When $\left[\mathrm{Ca}^{2+}\right]_{\mathrm{i}}$ was elevated to around $10 \mu \mathrm{M}$ or even higher, a subsequent depolarizing pulse evoked almost no EPSC, indicating depletion of the releasable vesicle pool (Bollmann et al., 2000; Schneggenburger and Neher, 2000). Forskolin + IBMX induced a slight potentiation of the EPSC amplitudes (Fig. 3-4). The rapid increase in $\left[\mathrm{Ca}^{2+}\right]_{\mathrm{i}}$ by flash photolysis induced a small and slow outward current at the presynaptic terminal, which was not investigated further. The peak release rate and cumulative release increased only slightly when the control trace and the one following forskolin application were compared. The vesicle pool size was increased $1.13 \pm 0.05$-fold $(n=4)$.

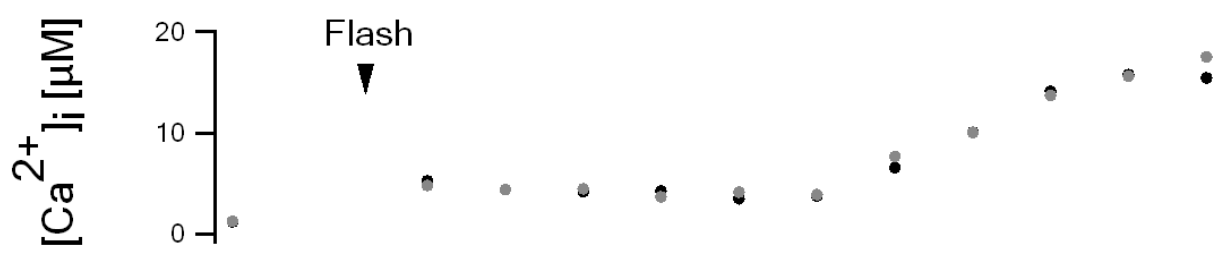

$\mathrm{I}_{\mathrm{Ca}}$

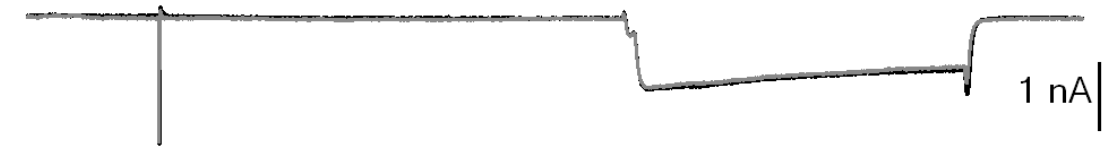

EPSC
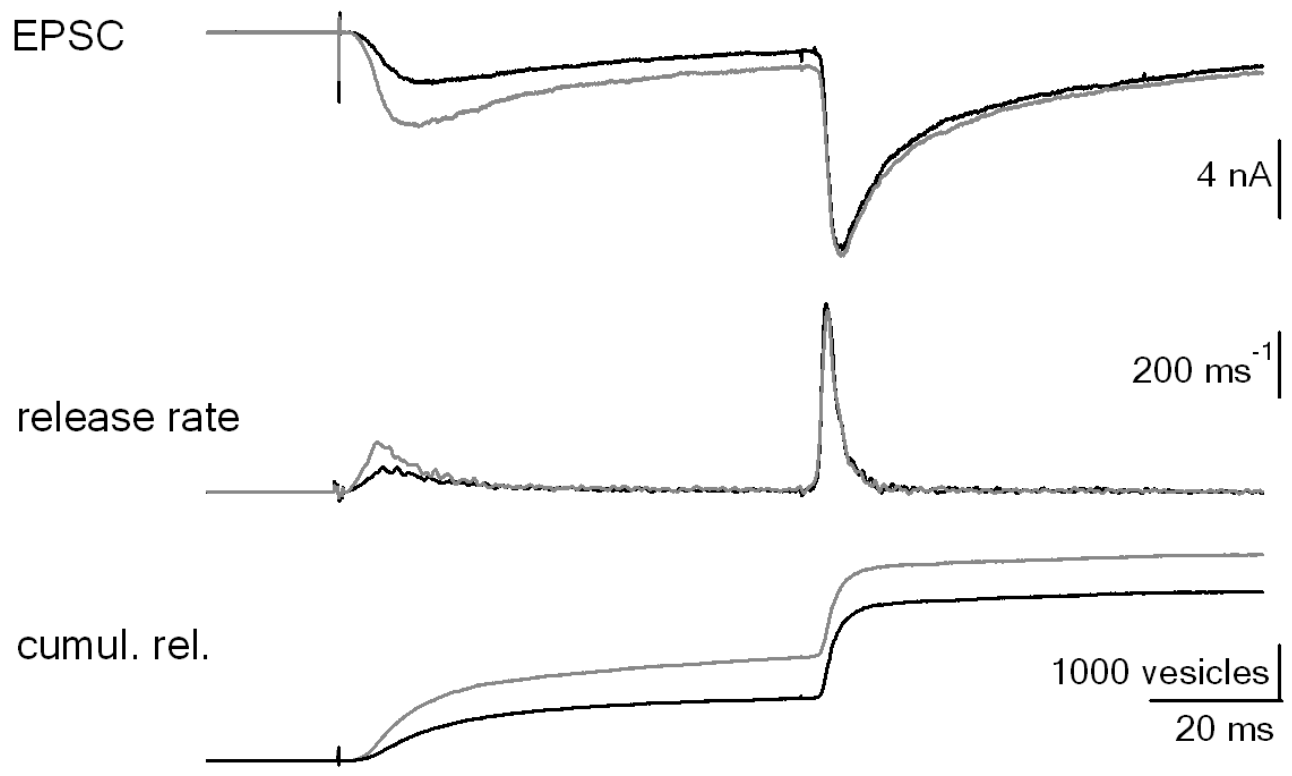

Figure 3-3: Forskolin increases transmitter release rates triggred by presynaptic $\mathrm{Ca}^{2+}$ uncaging at the calyx of Held synapse. 
The $\mathrm{Ca}^{2+}$-chelator DM-nitrophen and fluorescent $\mathrm{Ca}^{2+}$ indicator fura-2FF were loaded into the calyx terminal through presynaptic whole-cell dialysis. Intermediate intensity of flash photolysis was applied to elevate $\left[\mathrm{Ca}^{2+}\right]_{\mathrm{i}}$ uniformly within the terminal (the timing is indicated by the arrow). $60 \mathrm{~ms}$ after the flash photolysis, a step-depolarizing pulse was applied to deplete the remaining releasable pool of synaptic vesicles. From top to bottom, presynaptic $\left[\mathrm{Ca}^{2+}\right]_{i}, \mathrm{Ca}^{2+}$ currents, EPSCs, release rates and cumulative release are shown. The transient current seen in the $\mathrm{Ca}^{2+}$ currents and the EPSCs is the artefact caused by the fast flash lamp. In this example, the presynaptic $\left[\mathrm{Ca}^{2+}\right]_{\mathrm{i}}$ was increased from basal level to $5.27 \mu \mathrm{M}$ under control and $4.82 \mu \mathrm{M}$ after application of forskolin +IBMX.

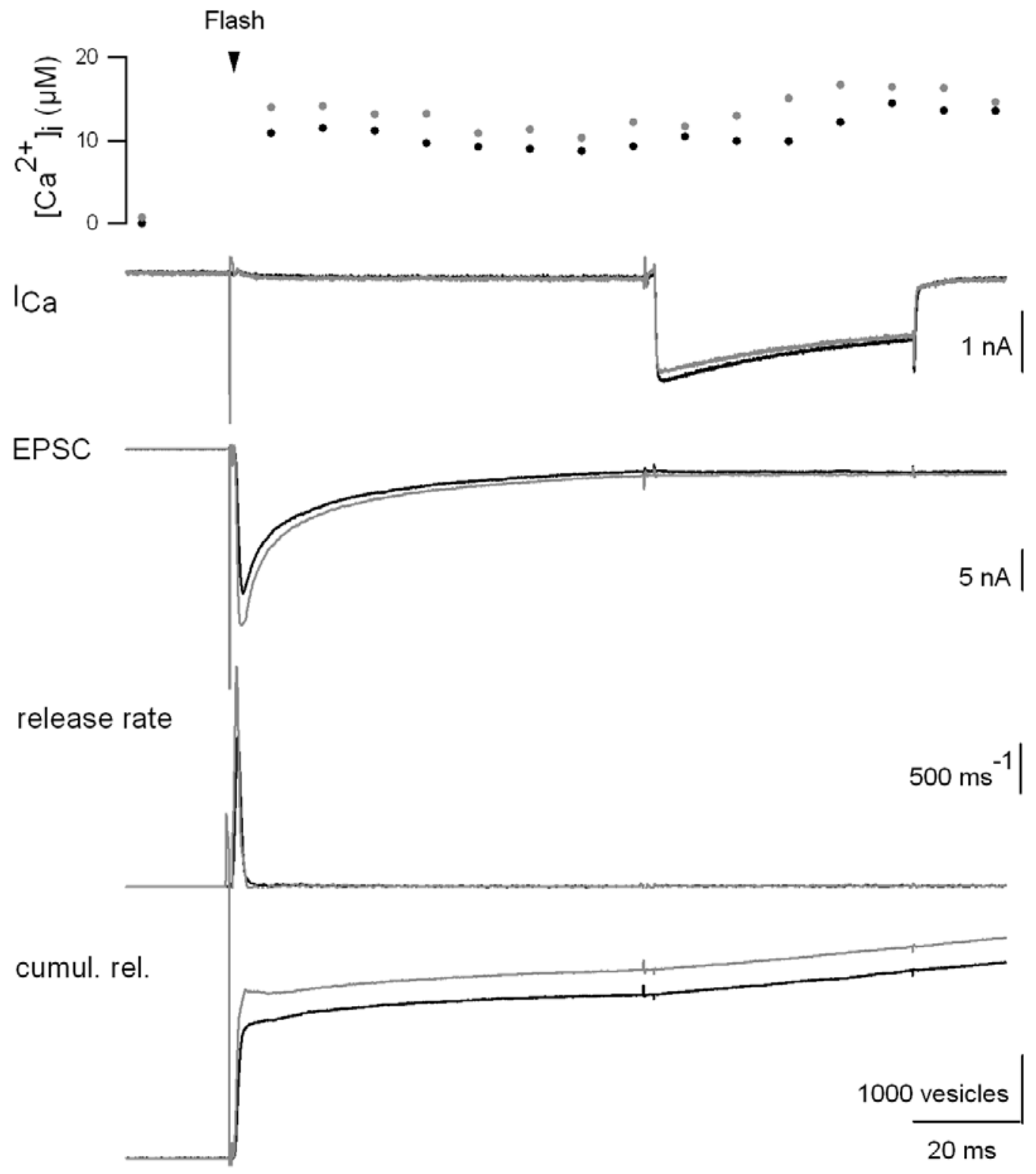


Figure 3-4: Forskolin potentiates relatively small amounts of potentiation of transmitter release rates in response to presynaptic $\mathrm{Ca}^{2+}$ uncaging to a high $\mathrm{Ca}^{2+}$ level.

Similar experimental procedure as Fig. 3-3 was applied to the terminal, but the presynaptic $\left[\mathrm{Ca}^{2+}\right]_{\mathrm{i}}$ was increased to $>10 \mu \mathrm{M}$.

Fig. 3-5 summarizes the $\mathrm{Ca}^{2+}$ uncaging experiments showing the peak transmitter release rates (shown as release rate per vesicle) as a function of $\left[\mathrm{Ca}^{2+}\right]$, both for the control condition and after application of forskolin + IBMX. Release rates per vesicle were calculated by dividing the peak release rate by the total size of the releasable vesicles (Wadel et al., 2007); in this way the error caused by the variability of the size of the releasable vesicle pool between different calyces is eliminated. Linear regression was applied to the whole data set after logarithmic transformation. The control data set (Fig. $3-5 A$ ) exhibited a slope of $4.43 \pm 0.37$. In contrast, the data obtained in the presence of forskolin + IBMX showed a slope of $3.07 \pm 0.27$, indicating that forskolin reduces the apparent $\mathrm{Ca}^{2+}$ cooperativity with which $\left[\mathrm{Ca}^{2+}\right]_{\mathrm{i}}$ induces vesicle fusion.

Synaptic delay is often used as a parameter to describe the kinetcis of synaptic vesicle fusion. Forskolin also affected the synaptic delay. In this study, synaptic delay was defined as the time between the triggering of the flash and a level crossing five released quanta in the cumulative release trace. Then the data were plotted as a function of $\left[\mathrm{Ca}^{2+}\right]_{\mathrm{i}}$ (Fig. 3-5B). The synaptic delay was shortened after the application of forskolin + IBMX for $\left[\mathrm{Ca}^{2+}\right]_{\mathrm{i}}$ steps below $7 \mu \mathrm{M}$, indicating that cAMP accelerated transmitter release kinetics. Overall, in the $\left[\mathrm{Ca}^{2+}\right]_{\mathrm{i}}$ range of $2-14 \mu \mathrm{M}$, the synaptic delays were always $<10 \mathrm{~ms}$. This indicates that $\mathrm{Ca}^{2+}$ can bind to the $\mathrm{Ca}^{2+}$ sensor in this synapse very rapidly, which is consistent with other reports (Bollmann et al., 2000; Schneggenburger and Neher, 2000). Taken together, the results suggest that when the concentration of cAMP is elevated in the presynaptic terminal, the intracellular $\mathrm{Ca}^{2+}$ sensitivity of vesicle fusion increases after flash photolysis, especially at lower $\mathrm{Ca}^{2+}$ micromolar range concentrations. 
A

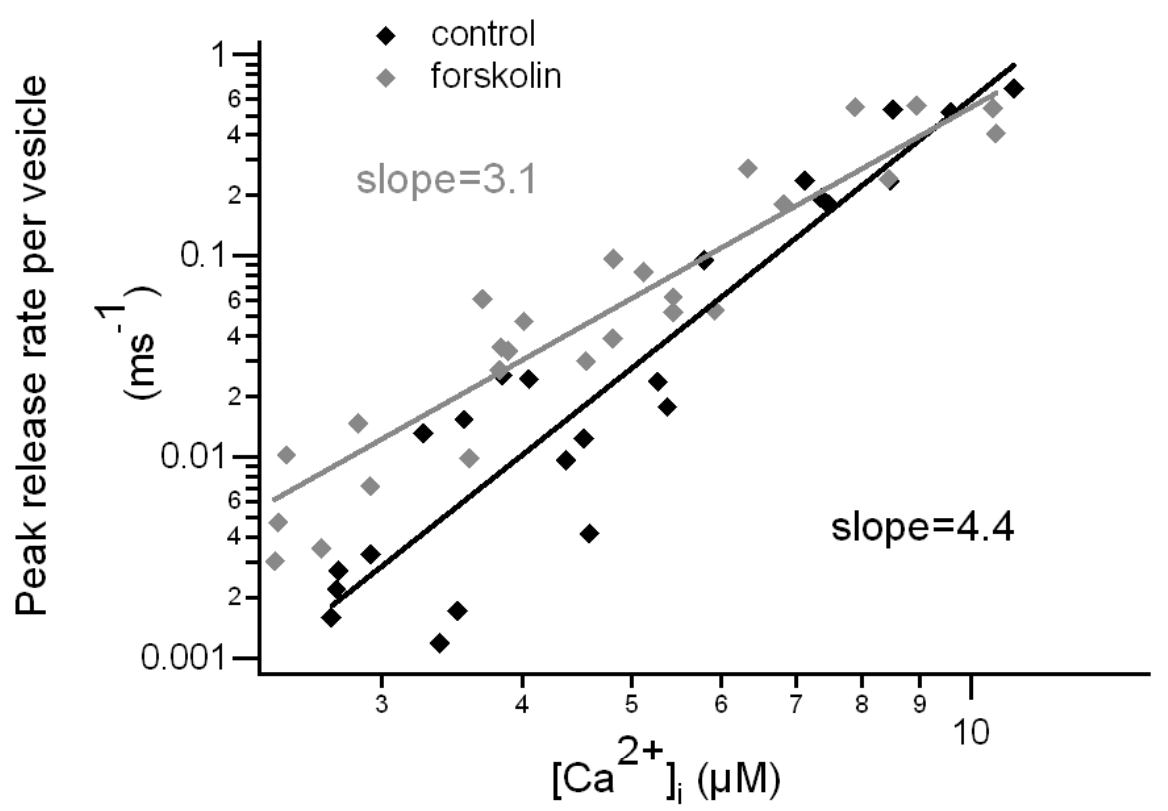

B

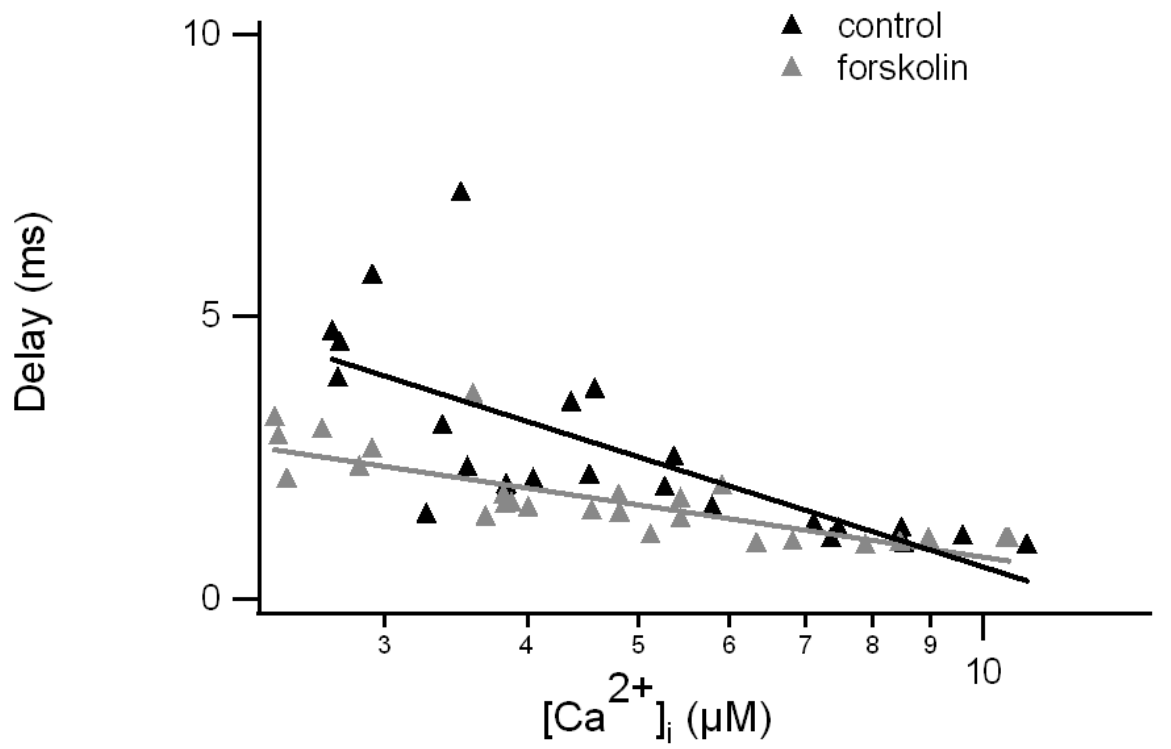

Figure 3-5: Relationship of the rates of synaptic vesicle fusion and synaptic delay as a function of $\left[\mathrm{Ca}^{2+}\right]_{\mathrm{i}}$.

A: Double-logarithmic plot of the peak release rate per vesicle evoked by flash photolysis as a function of presynaptic $\left[\mathrm{Ca}^{2+}\right]_{\mathrm{i}}$ under control (black) and forskolin-treated conditions (grey). The slope of the dose-response curve is 4.4 under control conditions, while it is 3.1 after forskolin application. $B$ : Synaptic delays were plotted as a function of $\left[\mathrm{Ca}^{2+}\right]_{\mathrm{i}}$, both in the control and forskolin-treated conditions. 


\subsection{3 cAMP increases transmitter release rates close to basal $\left[\mathrm{Ca}^{2+}\right]_{i}$}

cAMP increases the frequency but not the amplitude or kinetics of the miniature EPSCs (mEPSCs) (Kaneko and Takahashi, 2004; Sakaba and Neher, 2001b).The flash photolysis experiments showed that forskolin increased the intracellular $\mathrm{Ca}^{2+}$ sensitivity for vesicle fusion in response to submaximal stimuli of $\left[\mathrm{Ca}^{2+}\right]_{i}$. There is no experimental data showing the modulation of $\mathrm{Ca}^{2+}$ dependence of vesicle fusion by cAMP when the $\left[\mathrm{Ca}^{2+}\right]_{\mathrm{i}}$ is $<2 \mu \mathrm{M}$. The next experiment examined if forskolin affected transmitter release rates at lower $\left[\mathrm{Ca}^{2+}\right]_{\mathrm{i}}$ close to the basal level. To address this question, in the first set of experiments, $20 \mathrm{mM} \mathrm{EGTA} / \mathrm{Ca}^{2+}$ mixture solution was loaded to the calyx terminals via patch pipettes to clamp $\left[\mathrm{Ca}^{2+}\right]_{i}$ below $500 \mathrm{nM}$. Small amplitudes of leakage currents cannot be avoided even under tight-seal whole cell clamp. On the other hand, exogeneous free $\mathrm{Ca}^{2+}$ introduced via patch pipettes tends to be extruded to outside of the terminal. Therefore, the presynaptic $\left[\mathrm{Ca}^{2+}\right]_{i}$ was monitored with the high-affinity $\mathrm{Ca}^{2+}$ indicator fura $-4 \mathrm{~F}$, which has $\mathrm{K}_{\mathrm{d}}$ around $1 \mu \mathrm{M}$. Spontaneous mEPSCs were recorded during the initial 2-5 min after the formation of whole-cell configuration for control, and forskolin + IBMX was applied subsequently. In Fig. 3-6A, presynaptic $\left[\mathrm{Ca}^{2+}\right]_{i}$ was clamped around $130 \mathrm{nM}$. Forskolin + IBMX increased the frequency of spontaneous mEPSCs from 2.65 $\mathrm{Hz}$ to $10.3 \mathrm{~Hz}$ without a significant change in $\left[\mathrm{Ca}^{2+}\right]_{\mathrm{i}}$. When the clamped presynaptic $\left[\mathrm{Ca}^{2+}\right]_{\mathrm{i}}$ was increased, the mEPSC frequency also increased under control conditions but the $\mathrm{Ca}^{2+}$-dependence around this range of $\left[\mathrm{Ca}^{2+}\right]_{\mathrm{i}}$ was relatively shallow in the low $\mathrm{Ca}^{2+}$ range (see Fig. 3-8, for summary). On average, when the $\left[\mathrm{Ca}^{2+}\right]_{i}$ was below $500 \mathrm{nM}$, forskolin + IBMX increased mEPSC frequency $4.2 \pm 0.4$-fold. The potentiation of transmitter release was more prominent than that observed during a train of AP-like stimuli or in flash experiments, where the $\left[\mathrm{Ca}^{2+}\right]_{\mathrm{i}}$ levels was $>2 \mu \mathrm{M}$.

When more $\mathrm{Ca}^{2+}$ was added to the presynaptic solution, the $\left[\mathrm{Ca}^{2+}\right]_{\mathrm{i}}$ was increased apon whole-cell recording. It is very difficult to clamp $\left[\mathrm{Ca}^{2+}\right]_{\mathrm{i}}$ continuously around $1 \mu \mathrm{M}$ using an EGTA/ $\mathrm{Ca}^{2+}$ mixture because the relatively high rate of secretion causes rapid rundown of release, most likely arising from depletion of the recycling pool of vesicles. Therefore, in the next set of experiments, flash photolysis with DM-nitrophen but attenuated flash intensity with a neutral density filter, was used to produce a small increase of $\left[\mathrm{Ca}^{2+}\right]_{\mathrm{i}}(600 \mathrm{nM}-2 \mu \mathrm{M})$. Fura-4F was the $\mathrm{Ca}^{2+}$ indicator used in this 
experiment. At the same time, the UV excitation light from the monochromator was used to keep $\left[\mathrm{Ca}^{2+}\right]_{\mathrm{i}}$ stable for a few seconds, which also served for fluorescent
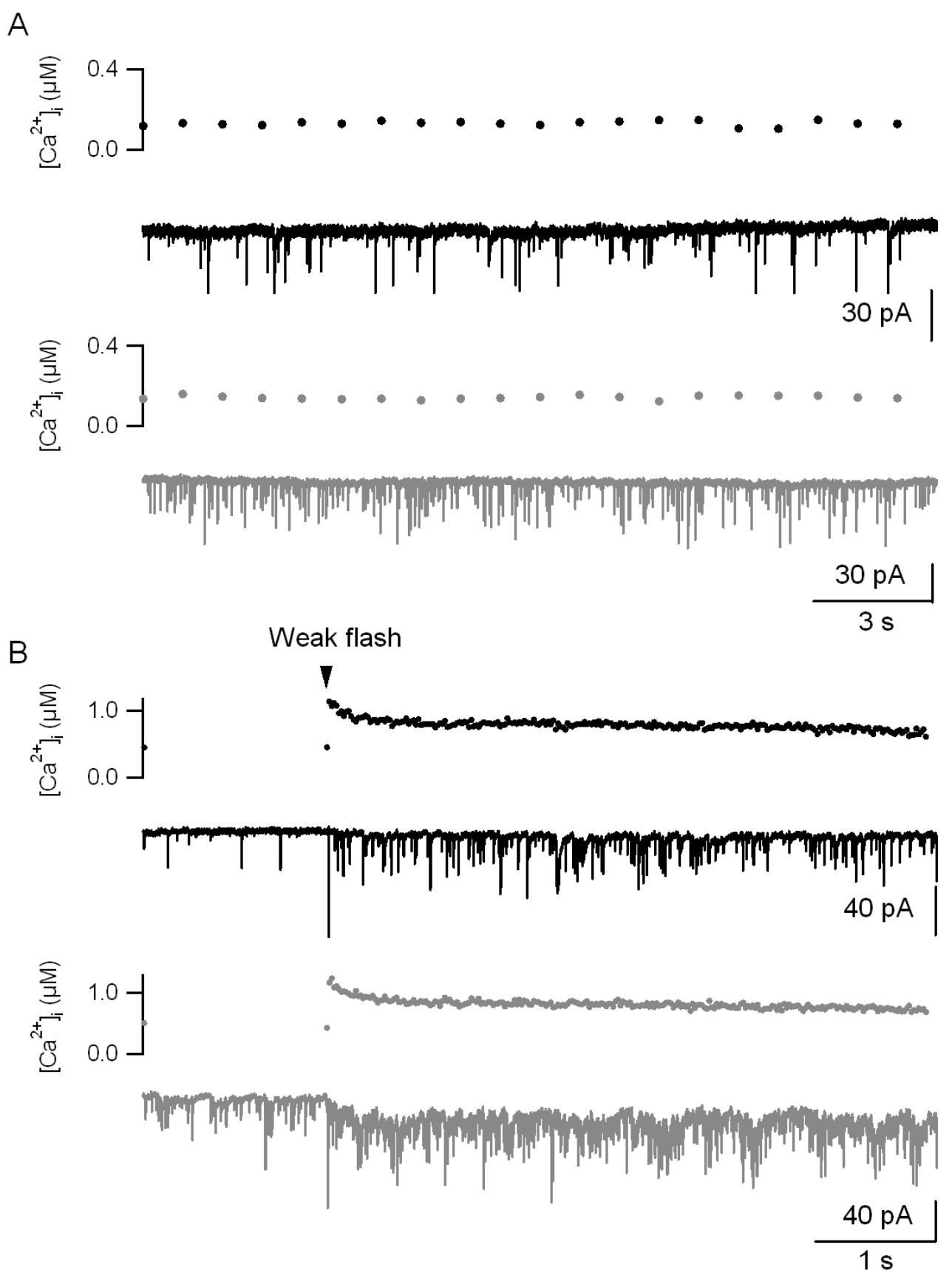

Figure 3-6: Forskolin increases the frequency of miniature EPSCs at low $\left[\mathrm{Ca}^{2+}\right]_{\mathrm{i}}$.

A: The presynaptic terminal was pre-loaded with an EGTA/ $\mathrm{Ca}^{2+}$ mixture and the $\mathrm{Ca}^{2+}$ indicator dye fura-4F. The $\left[\mathrm{Ca}^{2+}\right]_{\mathrm{i}}$ in presynaptic pipettes was clamped to $0-500 \mathrm{nM}$. Presynaptic $\left[\mathrm{Ca}^{2+}\right]_{\mathrm{i}}$ (upper) and postsynaptic mEPSCs (lower panel) were recorded simultaneously (black: control; grey: after 
forskolin application). B: Similar internal solution as for normal flash experiments was used, but 0.2 $\mathrm{mM}$ fura-4F was used instead of fura-2FF. A weak $\mathrm{Ca}^{2+}$-uncaging stimulus was applied at the time point indicated (arrow) to elevate $\left[\mathrm{Ca}^{2+}\right]_{\mathrm{i}}$ to a sub-micromolar level. The elevated $\left[\mathrm{Ca}^{2+}\right]_{\mathrm{i}}$ was comparative both in control and forskolin conditions. mEPSCs were recorded only in the last $4 \mathrm{~s}$ was used for further analysis.

measurement of $\left[\mathrm{Ca}^{2+}\right]_{i}$. The flash evoked mEPSCs were recorded during the first $2-5$ min after whole-cell formation for control as in previous experiments. Then forskolin + IBMX containing solution was applied subsequenty. Fig. 3-6B shows a typical example of such an experiment. Flash photolysis elevated $\left[\mathrm{Ca}^{2+}\right]_{\mathrm{i}}$ to around $900 \mathrm{nM}$ both in control and forskolin + IBMX conditions in this cell pair. After flash photolysis, the $\left[\mathrm{Ca}^{2+}\right]_{\mathrm{i}}$ was initially higher than the late phase, but it became stable in the following $20-30 \mathrm{~s}$. The frequency of mEPSCs was measured for the last $4 \mathrm{~s}$, where the $\left[\mathrm{Ca}^{2+}\right]_{\mathrm{i}}$ was relatively stable. Forskolin + IBMX markedly increased the mEPSC frequency by 3.75 -fold in this example, for summary see Fig. 3-8A.

\subsubsection{Fast- but not slowly-releasing vesicles are modulated by cAMP}

At the calyx of Held synapse, two kinetically different components of transmitter release have been described during a prolonged depolarization (Sakaba and Neher, 2001). The fast component of release has a time constant of 2-3 ms and a slow component has the time constant of 10-30 ms, respectively. This may result from differences in proximities of the two sets of vesicles to the $\mathrm{Ca}^{2+}$ channel clusters (Wadel et al., 2007). It has been shown that the fast-releasing vesicles, but not slowly releasing vesicles, are sensitive to cAMP at the calyx of Held (Sakaba and Neher, 2001b). During flash photolysis, all the vesicles "see" the same concentration of $\mathrm{Ca}^{2+}$, and it is difficult to separate between the fast- and slowly-releasing synaptic vesilces. In addition, phorbol esters, which are involved in the $\mathrm{PKC} /$ munc13 pathway cascades have a similar effect to that of cAMP described here, seem to potentiate all the vesicles equally (Lou et al., 2005). It is possible that cAMP accelerates the release time constant of all the vesicles equally and results in faster vesicle pool depletion. However, the pool depletion is compensated by the increase in number of releasable vesicles. As a result, the slow component of 
transmitter release appears to be unchanged, which however does not imply that cAMP does not modulate slowly releasing vesicles.

A
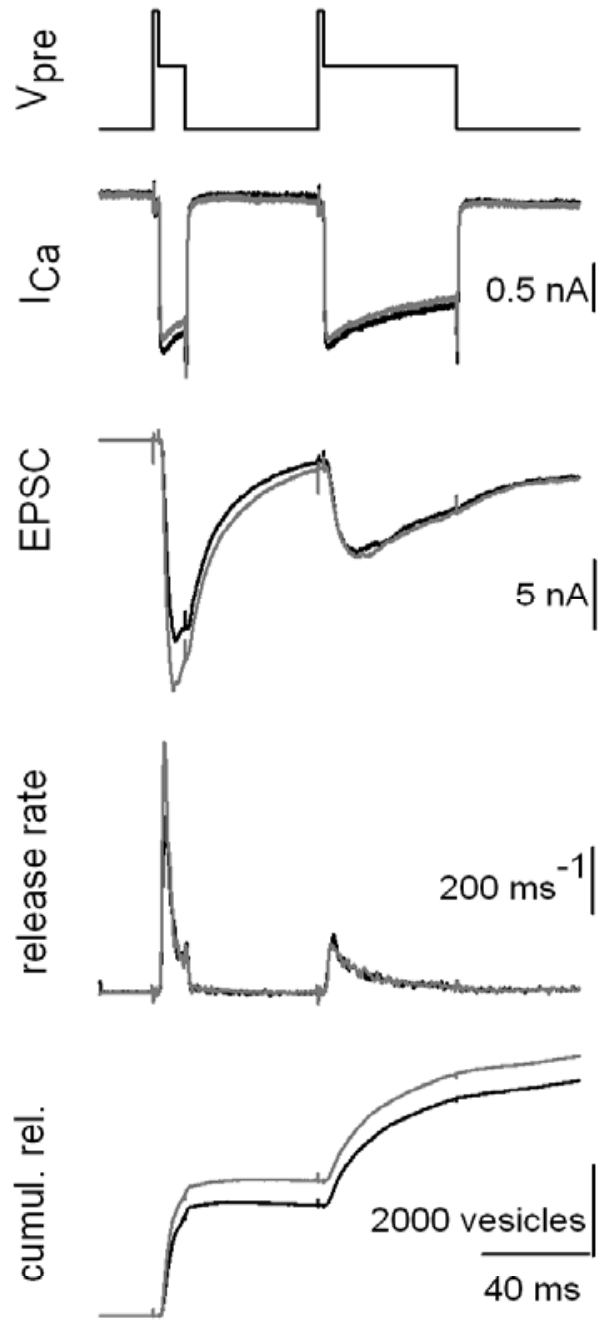

B
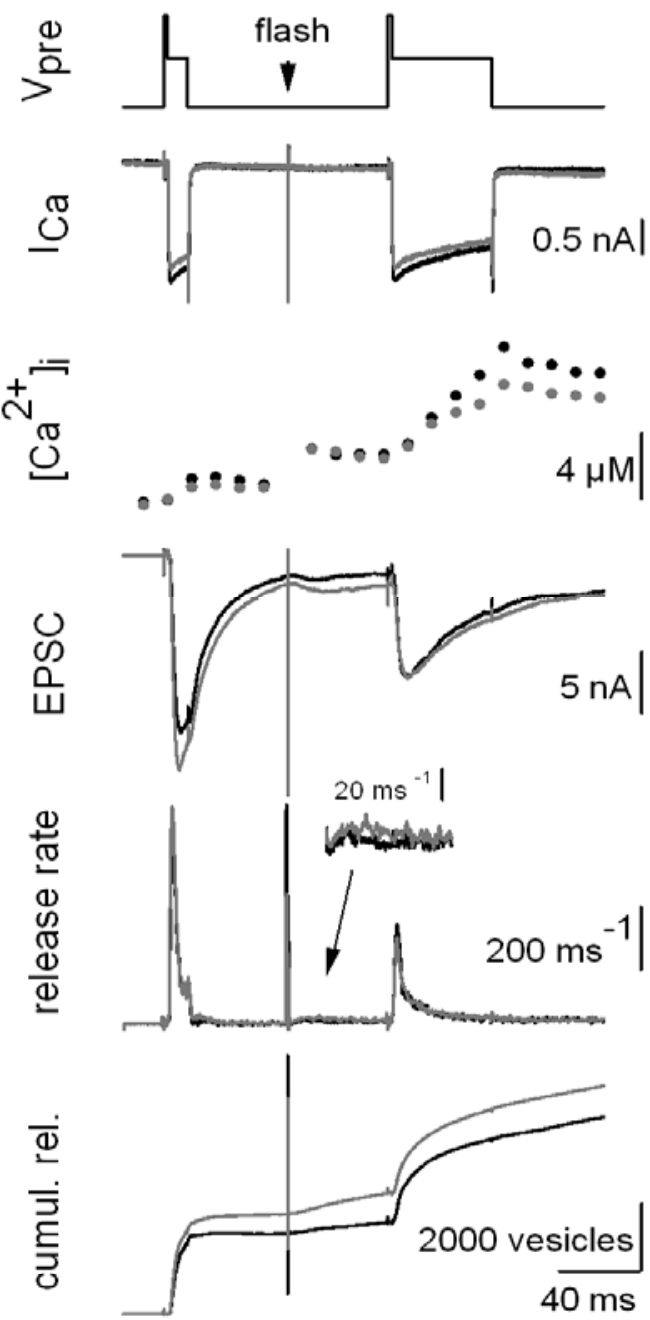

C

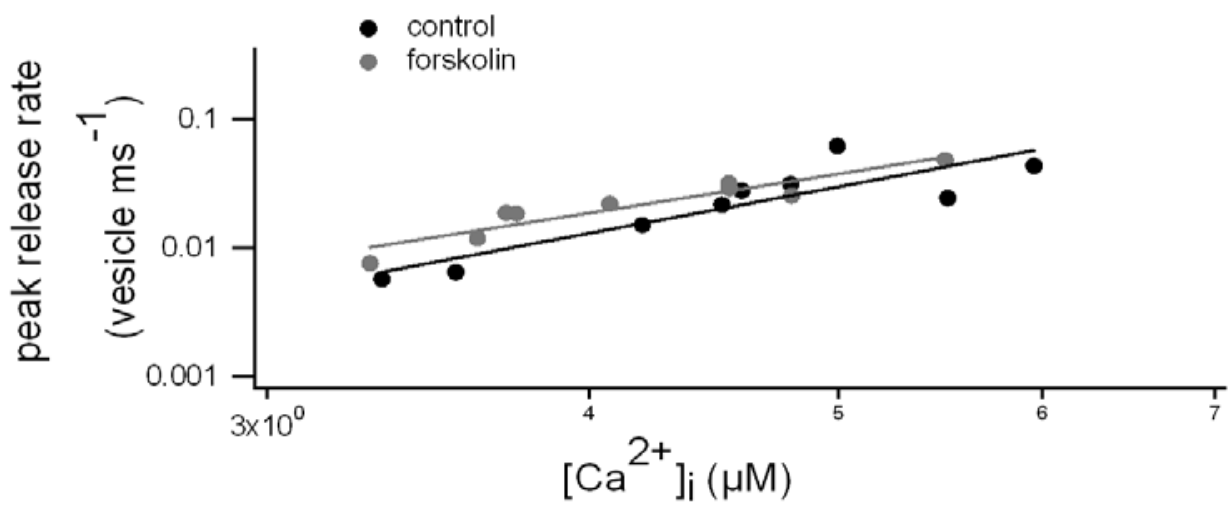


Figure 3-7: cAMP modulates the fast-releasing vesicles but not the slowly-releasing vesicles during flash photolysis.

$A$ : The fast-releasing vesicles were depleted by a pre-depolarizing pulse $(0 \mathrm{mV}$ for $10-15 \mathrm{~ms}$ following a pre-pulse to $+70 \mathrm{mV}$ for $2 \mathrm{~ms}$ ). Following the pre-depolarizing pulse, the test pulse was applied ( 0 $\mathrm{mV}$ for $50 \mathrm{~ms}$ following a pre-pulse to $+70 \mathrm{mV}$ for $2 \mathrm{~ms}$ ) to deplete the remaining vesicles. From top to bottom, presynaptic voltage command, presynaptic $\mathrm{Ca}^{2+}$ currents, EPSCs, the transmitter release rates estimated by the deconvolution method, and the cumulative amounts of release are shown. Black and gray traces indicate those obtained during the control period and after the application of forskolin + IBMX, respectively. $20 \mu \mathrm{M}$ calmodulin inhibitory peptide was included in the presynaptic patch pipette to ensure that rapid, calmodulin-dependnet recovery of synaptic vesicles was minimized. $B$ : The same as $A$, but instead of the test pulse, flash photolysis was applied to probe the intracellular $\mathrm{Ca}^{2+}$ sensitivity of the slowly-releasing vesicles. Following flash photolysis, a depleting pulse was applied to deplete the remaining synaptic vesicles. $C$ : The relationship between the peak release rate per vesicle of the slowly releasing vesicles and $\left[\mathrm{Ca}^{2+}\right]_{\mathrm{i}}$ under control (black circles) and in the presence of forskolin + IBMX (gray circles) from the experiment shown in $B$.

In order to address this question, in the next experiments, fast-releasing synaptic vesicles were depleted, and subsequently, the remaining synaptic vesicles were released by flash photolysis. This protocol was used to examine whether cAMP modulated the intracellular $\mathrm{Ca}^{2+}$ sensitivity of the slowly-releasing vesicles. Specifically, a short depolarizing pulse (depolarization to $0 \mathrm{mV}$ for $10-15 \mathrm{~ms}$ following a pre-pulse to +70 $\mathrm{mV}$ for $2 \mathrm{~ms}$ ) was used to release all the fast-releasing vesicles, and a subsequent test pulse (50 ms depolarization pulse following a pre-pulse to $+70 \mathrm{mV}$ for $2 \mathrm{~ms}$ ) was applied to deplete the remaining slowly-releasing vesicles. In some experiments, calmodulin inhibitory peptide $(20 \mu \mathrm{M})$ was included in the presynaptic pipette solution to prevent recovery of the fast-releasing vesicles between the two pulses (Sakaba and Neher, 2001). Because the data were similar with and without the calmodulin inhibitory peptide, all of the data were pooled together. Fig. 3-7A shows a typical recording using the two-pulse protcol. The second, test pulse evoked a more slowly rising EPSC. Accordingly, cumulative release also showed a slow increase, indicating that only slowly-releasing vesicles were released during the test pulse. Forskolin + IBMX only potentiated the EPSC during the pre-pulse, but not during the test pulse, confirming previous results 
(Sakaba and Neher, 2001b). The amplitude of $\mathrm{Ca}^{2+}$ currents did not change after aplication of forskolin + IBMX, which is similar to Fig. 3-1.

Next, flash photolysis was applied instead of the test pulse for probing the intracellular $\mathrm{Ca}^{2+}$ sensitivity of the slowly-releasing vesicles (shown in Fig. 3-7B). Flash intensity was adjusted so that $\left[\mathrm{Ca}^{2+}\right]_{\mathrm{i}}$ was increased to 3-6 $\mu \mathrm{M}$, at which level the cAMPinduced potentiation of release was relatively large with a stand-alone flash photolysis (Fig. 3-3). As Fig. 3-7B shows, the 10-15ms pre-pulse potentiated EPSCs but the flashevoked EPSCs were not modulated significantly (Fig. 3-7B), indicating little modulation of the slowly releasing vesicles by cAMP. Fig. 3-7C shows the summary of the relationship between release rate per vesicle of the slowing-releasing vesicle and $\left[\mathrm{Ca}^{2+}\right]_{i}$. Under control conditions, release rates per vesicle of the slowly-releasing vesicles were the same as the fast-releasing component (compare Figs. 3-5 and 3-7), indicating that the two components were equally release-competent when $\left[\mathrm{Ca}^{2+}\right]_{i}$ was elevated uniformly. Unlike the outcome depicted in Fig. 3-5, however, forskolin +IBMX did not potentiate the release rate per vesicle. The results indicate that only the intracellular $\mathrm{Ca}^{2+}$ sensitivity of the fast-releasing vesicles was modulated.

\subsubsection{Modelling the Ca2+-dependent synaptic vesicle fusion with a simplified}

\section{allosteric model}

In order to observe the modulation of the $\mathrm{Ca}^{2+}$-dependent vesicle fusion by cAMP more clearly and to address the underlying mechanisms, a simplified $\mathrm{Ca}^{2+}$-binding model was used. Data obtained from $\left[\mathrm{Ca}^{2+}\right]$ i-clamp experiments, weak flashes, and normal flashes were pooled together and the peak release rate per vesicle was plotted against $\left[\mathrm{Ca}^{2+}\right]$. The release rates per vesicle were calculated by dividing the peak transmitter release rate by the number of readily-releasable synaptic vesicles, which includes both the fast- and the slowly releasing vesicles. This procedure is the same as that used previously (Wadel et al., 2007). If the slowly releasing vesicles were excluded, the release rates per vesicle would be 2 -fold higher, but the overall conclusions described below remain the same. 
The $\mathrm{Ca}^{2+}$ cooperativity of vesicle fusion gradually decreased at lower $\left[\mathrm{Ca}^{2+}\right]_{\mathrm{i}}$ under control conditions (Lou et al., 2005). This result cannot be described by a classical $\mathrm{Ca}^{2+}$ binding model, which assumes that $\mathrm{Ca}^{2+}$ binds to the $\mathrm{Ca}^{2+}$ sensor of release sequentially and that vesicle fusion occurs exclusively from the fully occupied state (Bollmann et al., 2000; Schneggenburger and Neher, 2000). This sequential model predicts a release

A

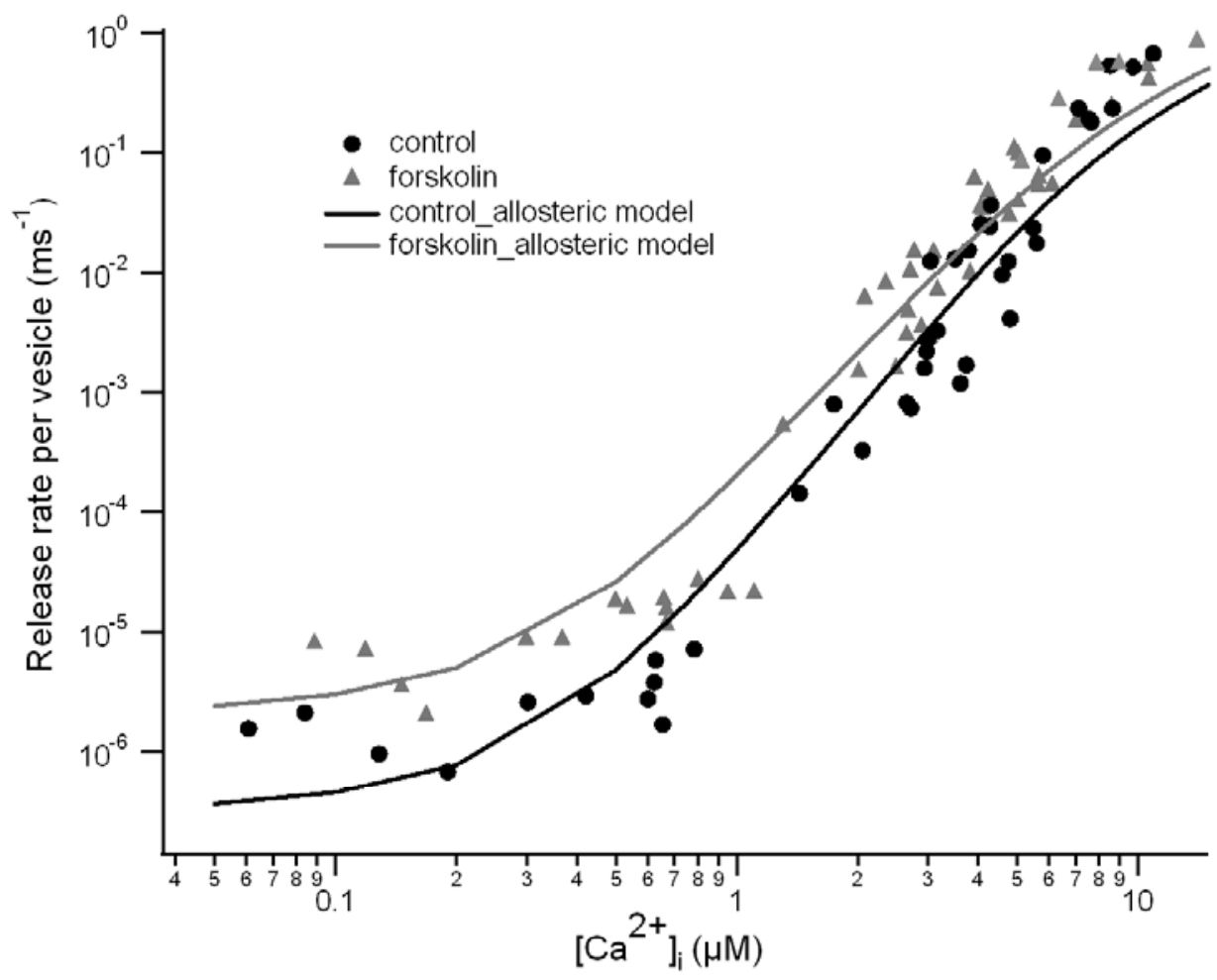

B

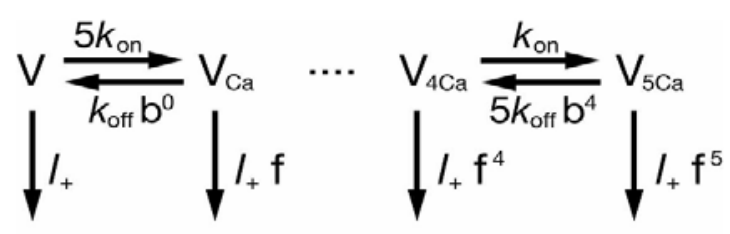

Fused

Figure 3-8: Simplified allosteric model of $\mathrm{Ca}^{2+}$-driven vesicle fusion.

A: Double-logarithmic plot of peak release rate per vesicle as a function of $\left[\mathrm{Ca}^{2+}\right]_{\mathrm{i}}$. The data were obtained in $\mathrm{Ca}^{2+}$ perfusion experiments $(10 \mathrm{nM}$ to $500 \mathrm{nM})$, weak $\mathrm{Ca}^{2+}$-uncaging stimulus ( $600 \mathrm{nM}$ to 
$1.5 \mu \mathrm{M})$ with fura-4F and normal flash photolysis with fura2-FF (2 $\mu \mathrm{M}$ to $14 \mu \mathrm{M})$. The black (control) and grey (forskolin) lines were predictions from the simplified allosteric model. All the parameters are as follows: control: $\mathrm{k}_{\mathrm{on}}\left(\mathrm{M}^{-1} \mathrm{~s}^{-1}\right)=1.5 \mathrm{e}^{*} 10^{8}, \mathrm{k}_{\mathrm{off}}\left(\mathrm{s}^{-1}\right)=6000,1_{+}\left(\mathrm{s}^{-1}\right)=3 * 10^{-4}, \mathrm{~b}=0.5, \mathrm{f}=30$; forskolin: $\mathrm{k}_{\text {on }}\left(\mathrm{M}^{-1} \mathrm{~s}^{-1}\right)=1.5 \mathrm{e}^{*} 10^{8}, \mathrm{k}_{\text {off }}\left(\mathrm{s}^{-1}\right)=6000,1_{+}\left(\mathrm{s}^{-1}\right)=2 * 10^{-3}, \mathrm{~b}=0.5, \mathrm{f}=30 . \quad B$ : The scheme of the simplified allosteric model.

rate of 0 at zero $\left[\mathrm{Ca}^{2+}\right]$, which is not the case for the experimental data (Fig. 3-6). Alternatively, the data were fitted with a simplified allosteric model (Lou et al., 2005), in which the rate $l_{+}$allows low rates of vesicle fusion in the absence of bound $\mathrm{Ca}^{2+}$ and increasingly higher rates of vesicle fusion are attained when the $\mathrm{Ca}^{2+}$ sensor is more completely occupied by $\mathrm{Ca}^{2+}$ (Fig. 3-8B). For control data, the parameters were determined as follows: $\mathrm{k}_{\mathrm{on}}=1.5 \times 10^{-8} \mathrm{M}^{-1} \mathrm{~s}^{-1}, \mathrm{k}_{\text {off }}=6000 \mathrm{~s}^{-1}, \mathrm{l}_{+}=3 \times 10^{-4} \mathrm{~s}^{-1}, \mathrm{f}=30, \mathrm{~b}=$ 0.5. The forskolin + IBMX-treated data could be fitted by changing $l_{+}$to $2 \times 10^{-3} \mathrm{~s}^{-1}$, without changing other parameters. According to the model, forskolin + IBMX increases the basal fusion 'willingness', similar to the effect of phorbol esters at the calyx of Held (Lou et al., 2005). The increase in $l_{+}$predicts enhanced fusion rates not only at basal $\left[\mathrm{Ca}^{2+}\right]_{\mathrm{i}}$ but also at intermediate $\left[\mathrm{Ca}^{2+}\right]_{\mathrm{i}}(2-7 \mu \mathrm{M})$. 


\section{2 cAMP modulates the rate of endocytosis}

After exocytosis, fused presynaptic membrane undergoes retrieval for recycling in order to maintain a steady-state synaptic transmission. Block of endocytosis leads to an eventual loss of synaptic vesicle fusion due to depletion of the reserve vesicles. The time course of membrane retrieval critically depends on the amount of exocytosis. cAMP can modulate the synaptic transmission by changing the intracellular $\mathrm{Ca}^{2+}$ sensitivity for vesicle fusion. So far, it is not clear if second messengers such as cAMP also participate in the process of endocytosis at the presynaptic terminal. In the next part of study, I will address this question by presynaptic capacitance measurement, which reflects the amount and kinetics of the membrane dynamics.

\subsubsection{Normal endocytosis at the calyx of Held synapse}

The presynaptic terminal was patch-clamped at $-80 \mathrm{mV}$ (from P9 to P11 rats). Capacitance measurement was applied to monitor the kinetics of exo- and endocytosis. There are several forms of endocytosis operating in this giant terminal, depending on the strength of the stimulation. Normally, slow endocytosis, which takes tens of seconds, can be induced by mild stimulation. For fast endocytosis, membrane retrieval can be accomplished within several seconds, but it can only be induced by stronger stimulation. Before examining how endocytosis is modulated by second messengers, capacitance changes in response to different durations of the depolarizing pulse were monitored under control condition. In Fig. 3-9A left, a $50 \mathrm{~ms}$ depolarization pulse was applied to the terminal, which elicited a capacitance jump of $320 \mathrm{fF}$ (on average, $379 \pm 42 \mathrm{fF}$, see Table 3-1), reflecting exocytosis of around 5000 synaptic vesicles. Following exocytosis, capacitance decreased, which reflected membrane retrieval via endocytosis. At the same time, membrane conductance $(\mathrm{Gm})$ and series conductance $(\mathrm{Gs})$ were relatively stable. Membrane retrieval after the $50 \mathrm{~ms}$ pulse followed an half decay time (the time when the increased capacitance returns to the half value of the maximum jump) of 20-30 sec (Fig. 3-9A, left and Fig. 3-9B, for summary). The decay due to endocytosis was also fitted by a single or a double exponential, and the rates of endocytosis were calculated as the 
capacitance jump divided by the endocytosis time constant. With a $50 \mathrm{~ms}$ pulse, membrane retrieval occurred with a rate of $11.2 \pm 1.8 \mathrm{fF} / \mathrm{s}$ (Table 3-1 for summary). When a $500 \mathrm{~ms}$ depolarizing pulse was applied, a much larger capacitance jump was elicited (Fig. 3-9A, right). On average, a capacitance change of $1153 \pm 116 \mathrm{fF}$ was observed with this stimulation. Following exocytosis, a rapid component of endocytosis with a time constant of a few seconds was observed preceding the slow endocytosis (Fig. 3-9A right, Fig. 3-9B and Table 3-2 for summary). In order to observe the exodependence of endocytosis, different durations of depolarizing pulses or different numbers of AP-like stimuli at the frequency of $100 \mathrm{~Hz}$ were applied to the terminal. After all, capacitance jumps between $20 \mathrm{fF}$ to $1.64 \mathrm{pF}$ were obtained. To quantify the time course of endocytosis, half decay times were plotted against the amplitudes of capacitance jumps. Half decay time was used because endocytosis following strong stimulation tends to follow a bi-exponential time course. The data were binned according to the amounts of exocytosis. As seen in Fig 3.9B, the endocytotic time course became progressivley as the amount of exocytosis increased up to the capacitance jump of $600 \mathrm{fF}$. This is consistent with the limited capacity of clathrin mediated endocytosis. However, stronger stimulation accrelates the time course of endocytosis because of the apperance of rapid endocytosis. Nevertheless, the time course of endocytosis was similar to that of previous studies (Hosoi et al., 2009; Wu et al., 2005; Yamashita et al., 2005). I also fitted the data with a double exponetial, following $500 \mathrm{~ms}$ pulse, membrane retrieval occurred with a fast endo-rate of $145 \pm 23 \mathrm{fF} / \mathrm{s}$ and a slow endo-rate of $36.0 \pm 7.9 \mathrm{fF} / \mathrm{s}$, respectively (see Table 3-2). So far, I have obtained the kinetics of endocytosis at the calyx of Held synapse under control condition. Next, different chemical reagents and peptides were introduced into the pipette solution or applied extracellularly in order to probe the function of cAMP during endocytosis. 
A

$50 \mathrm{~ms}$ pulse

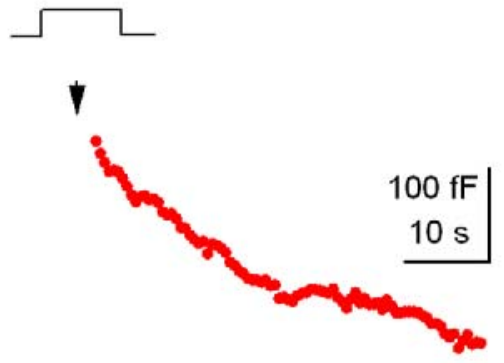

$\mathrm{Cm}$
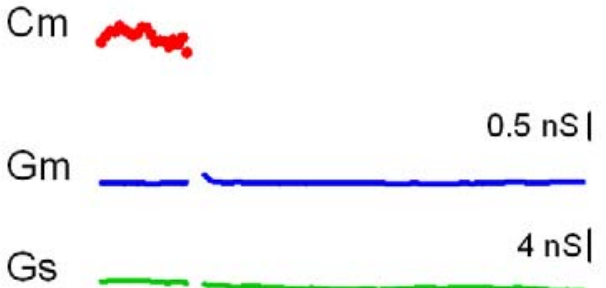

$500 \mathrm{~ms}$ pulse

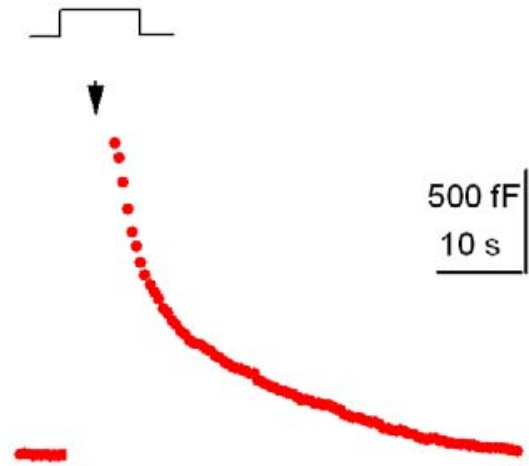

$0.5 \mathrm{nS} \mid$

$4 \mathrm{nS} \mid$

B

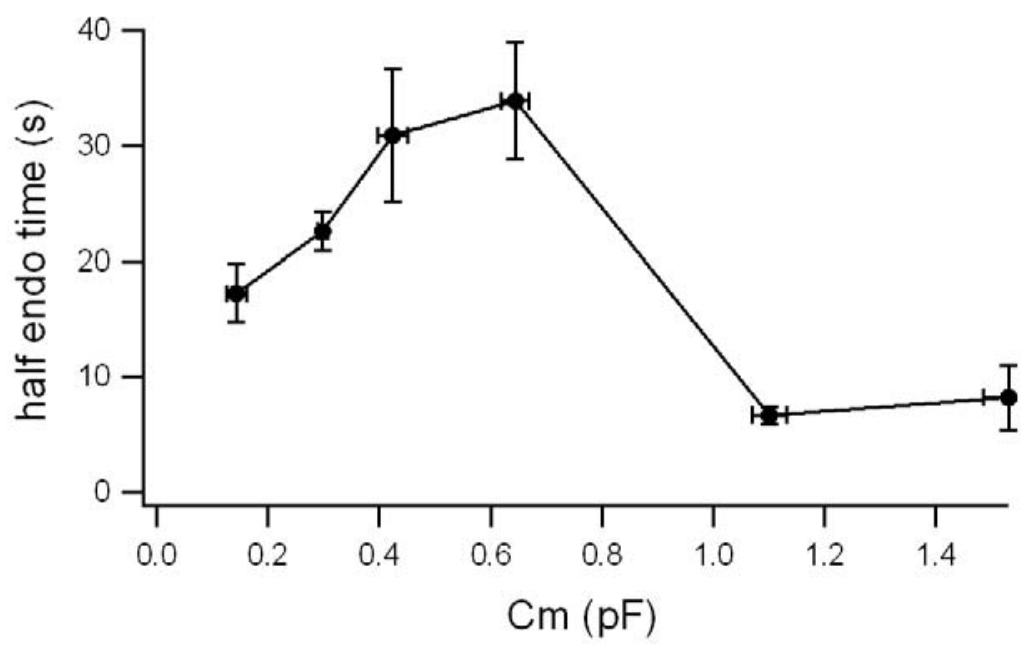

Figure 3-9: Fast and slow forms of endocytosis co-exist at the calyx of Held synapse.

A: Presynaptic terminal was voltage-clamped at $-80 \mathrm{mV}, 50 \mathrm{~ms}$ (left) or $500 \mathrm{~ms}$ (right) depolarizing pulse (depolarized to $0 \mathrm{mV}$ ) was applied to the terminal. Membrane capacitance (Cm), membrane conductance $(\mathrm{Gm})$, and series conductance $(\mathrm{Gs})$ were recorded simutaneously. In the left panel, a 50 ms pulse induced a capacitance jump around $320 \mathrm{fF}$, and then the presynaptic membrane was retrieved with a half decay time of $21 \mathrm{~s}$. In response to a $500 \mathrm{~ms}$ depolarization pulse, a much larger capacitance jump was elicited, and in this case the maximal capacitance jump was $1.5 \mathrm{pF}$. Both fast and slow forms of endocytosis were observed with a half decay of $5 \mathrm{~s}$. B: In 25 terminals, different intensities of stimuli (either different numbers of APs in a train of AP-like stimuli with $100 \mathrm{~Hz}$ or different 
durations of step depolarization to $0 \mathrm{mV}$ ) were applied to the terminal. Capacitance jumps between 20 $\mathrm{fF}$ to $1.64 \mathrm{pF}$ were obtained. Half decay times of endocytosis were plotted against the capacitance jump. The data were binned according to the amounts of exocytosis. (Mean $\pm \mathrm{SEM}$, vertical bars indicated the SEM of half decay time and horizontal bars indicated the SEM of the summed capacitance jumps).

\subsubsection{Endogenous cAMP is essential for endocytosis following strong stimulation}

Extracellular application of forskolin, which increases the intracellular cAMP concentration, facilitates synaptic vesicle fusion. However, it is unclear about the function of cAMP in endocytosis. In order to address this question, $1 \mathrm{mM}$ cAMP was included in the presynaptic pipette solution. Following a $50 \mathrm{~ms}$ depolarization pulse, the capacitance jumps were not changed strongly with and without cAMP inside the patch pipettes; only a minor acceleration of endocytotic time constant was observed (Fig. 310A). Also, different intensities of stimuli (AP-like stimuli with $100 \mathrm{~Hz}$ or depolarization pulses, the same as Fig. 3-9) were applied to the calyx terminal. cAMP accelerated the rate of endocytosis when the capacitance jump was between 400 and $700 \mathrm{fF}$, but did not accerelate upon larger or weaker stimuli. Also, the effect was relatively mild, so the acceleration was less than 2-fold (Fig. 3-10B for summary). The reults suggest that (1) either cAMP has a minor modulatory role in endocytosis or else that (2) cAMP concentration is already sufficiently high in the terminal under control condition. 
A

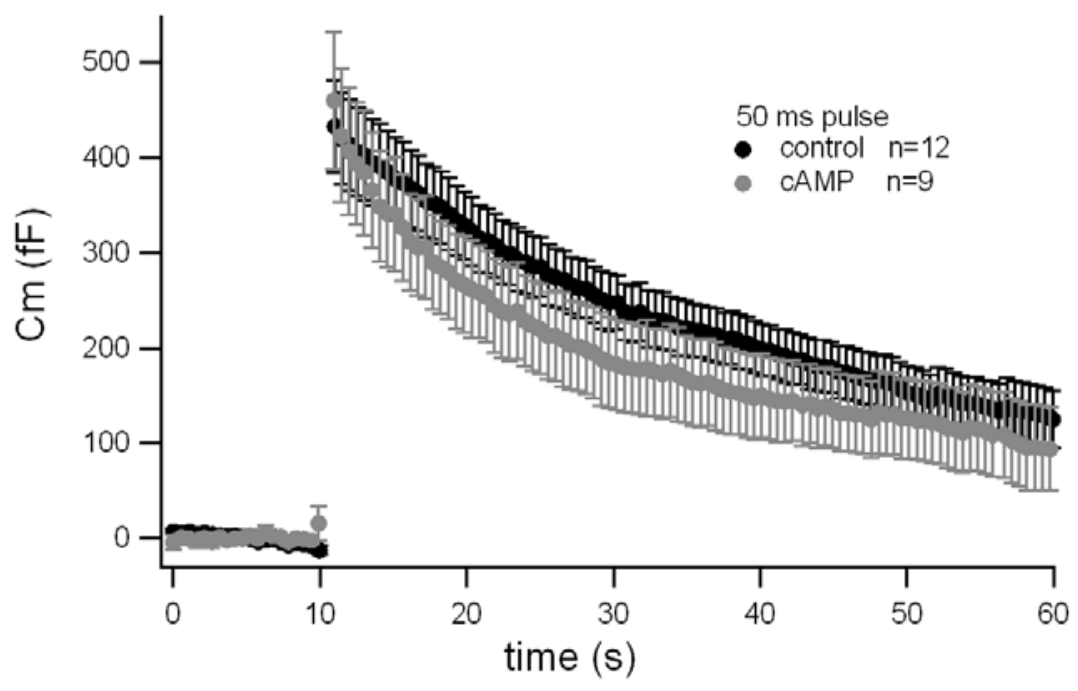

B

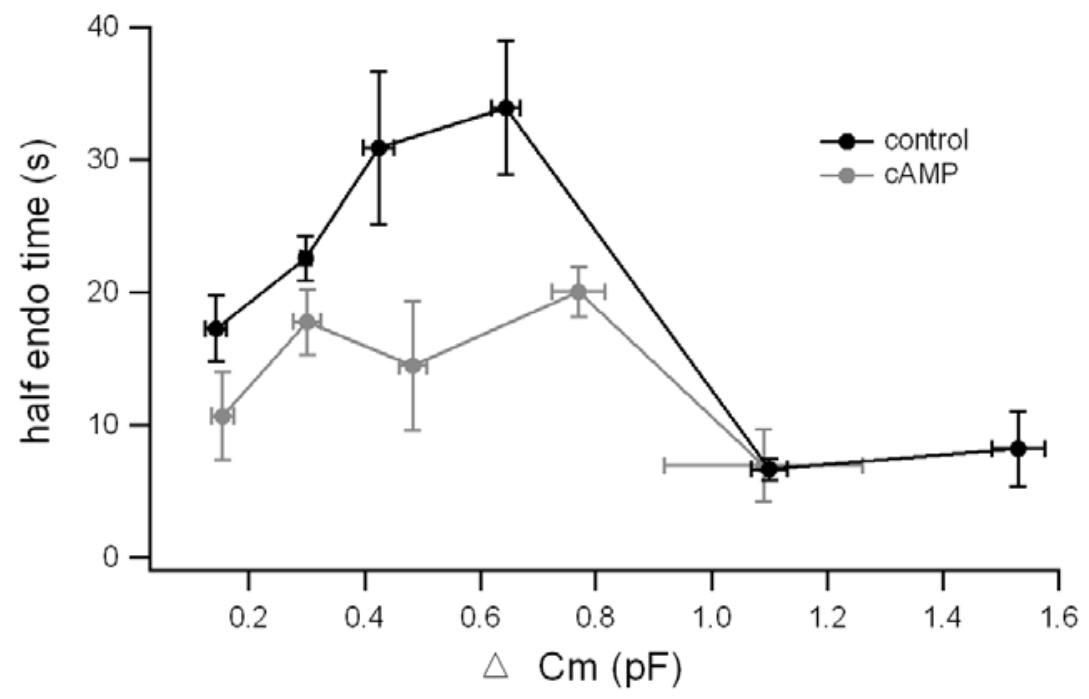

Figure 3-10: Elevation of presynaptic cAMP concentration has a mild effect on the rate of endocytosis.

A: Capacitance jumps were induced by a $50 \mathrm{~ms}$ depolarizing pulse. The average capacitance jumps under control condition (black circles) and in the presence of cAMP (1 mM, grey circles) were almost the same, but the half decay time of endocytosis in the presence of cAMP was slightly faster than that in control. B: Different intensities of stimuli (same as Fig. 3-9B) were applied to the terminal in the condition of cAMP. cAMP accelerated the rate of endocytos moderately following intermediate stimulation, which elicited exocytosis of 400-800 fF. (mean \pm SEM for half decay times of endocytosis and capacitance jumps, both for control and in the presence of cAMP). 
If the endogenous cAMP concentration is high and cAMP is important for endocytosis, suppressing cAMP level should slow down the rate of endocytosis. To test this possibility, $(50 \mu \mathrm{M}) \mathrm{MDL} 12330 \mathrm{~A}$, an inhibitor of adenylate cyclase, was extracellularly applied. In response to a $50 \mathrm{~ms}$ pulse (Fig. 3-11A), capacitance decayed relatively normally, or if any, MDL 12300A modulated endocytosis only moderately (See Fig. 3-12 for summary). In response to a long depolarizing pulse (500 ms), endocytosis was significantly slowed by MDL 12330A. Because MDL 12330A reduced Ca influx during the depolarization, less capacitance jump was elicited. In order to compare with the exocytosis in control conditions, even longer stimulation (1s depolarization) was applied to the terminal. Large capacitance jump was induced as seen in Fig 3-11C, and endocytosis was slowed down as that upon the $500 \mathrm{~ms}$ pulse. The data suggest that basal cAMP level is high and maintains normal endocytosis during strong stimulation at the calyx of Held. In contrast, endocytosis following weak stimulation is moderately or not modulated by cAMP, suggesting that this modulation is activity-dependent. 
A

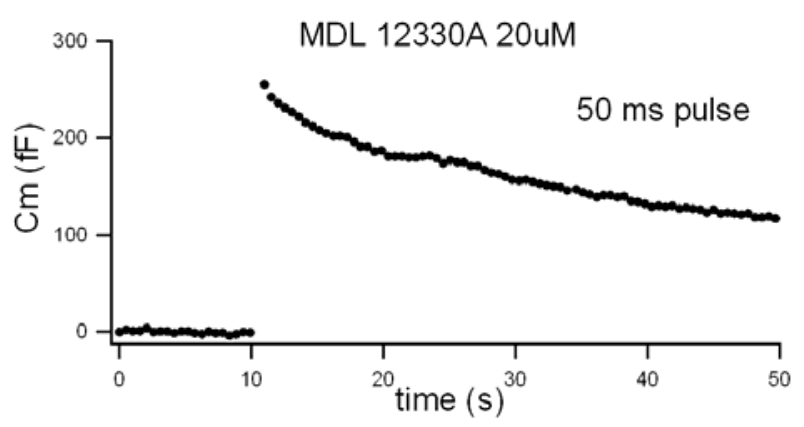

$\mathrm{B}$

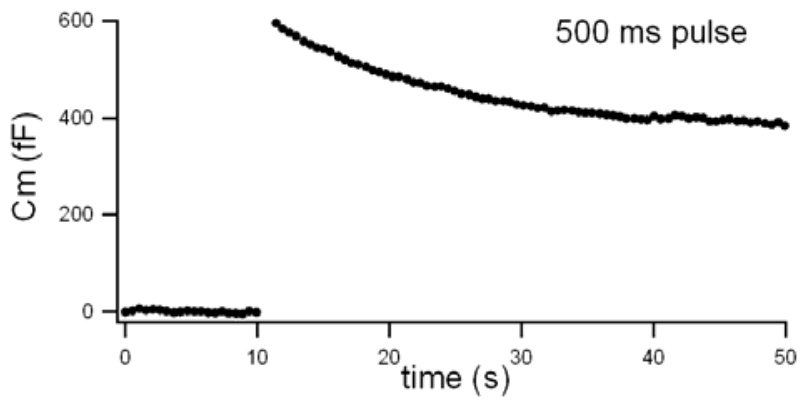

C

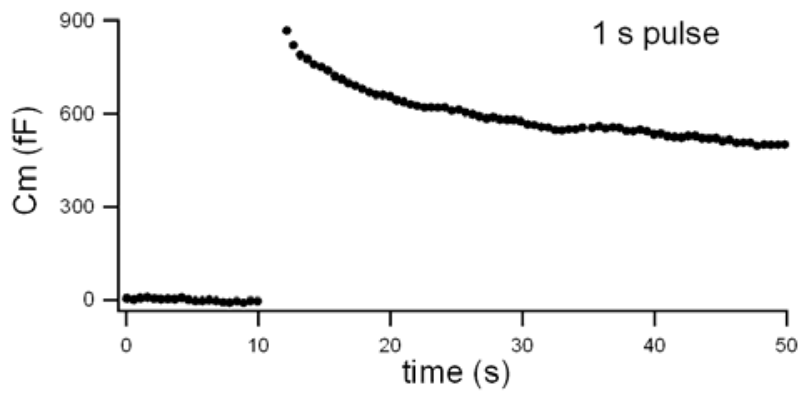

Figure 3-11: Modulation of endocytosis by an adenylate cyclase inhibitor, MDL 12330A. Capacitance traces in response to $50 \mathrm{~ms}(A), 500 \mathrm{~ms}(B)$ and $1 \mathrm{~s}(C)$ pulses are shown, similarly to Fig. 3-10. The data were obtained in the presence of $50 \mu \mathrm{M}$ MDL 12330A in the extracellular solution.

Epac and PKA are the two classical downstream targets for cAMP. It has been shown that Epac but not PKA is involved in the modulation of vesicle fusion (Kaneko and Takahashi, 2004; Sakaba and Neher, 2001b). Next, the possible downstream target of cAMP in endocytosis was tested. The brainstem slice was incubated with KT5720 (2 $\mu \mathrm{M}$ ), an inhibitor of protein kinase A (PKA) for 30-60 mins at room temperature. Similar to MDL 12330A, endocytosis was slowed down significantly under KT 5720 following a 
$500 \mathrm{~ms}$ pulse, while the effect was only moderate in response to a $50 \mathrm{~ms}$ pulse (Fig. 312A, B,red circles, also see Table 3-1, 3-2). Due to the lack of availability, we couldn't try the inhibitor of Epac in our experiments. Instead we did put calmodulin inhibitory peptide together with Epac agonist 8-CPT-2Me-cAMP into the patch pipette (we hypothesized cAMP is the downstream effector of calmodulin, see later). 8-CPT-2MecAMP could not reverse the inhibition caused by calmodulin inhibitor, but PKA agonist 6-Bnz-cAMP could reverse it (see also Fig. 3-16). All together, these results suggested that cAMP/PKA, but not Epac was involved in endocytosis during strong stimulation at the calyx of Held.
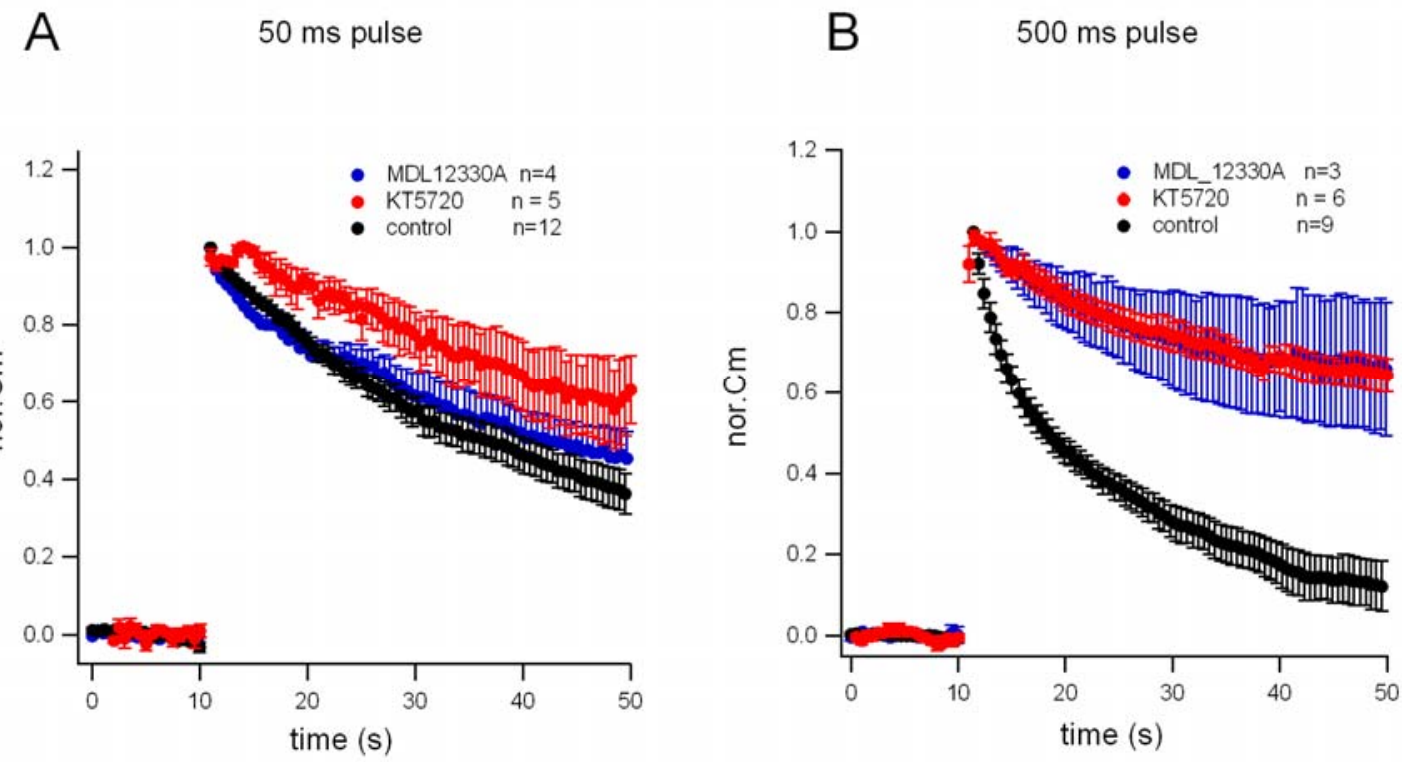

Figure 3-12: Modulation of endocytosis by an adenylate cyclase inhibitor and a PKA inhibitor. $50 \mathrm{~ms}(A)$ or $500 \mathrm{~ms}(B)$ depolarizing pulses were applied to the calyx of Held terminal both in control conditions, in the presence of $50 \mu \mathrm{M}$ MDL 12330A, or $2 \mu \mathrm{M}$ KT 5720. The data were normalized to the maximal capacitance jump obtained in each trace during the stimulation. On average, in response to a $50 \mathrm{~ms}$ pulse, the rate of endocytosis was almost the same among control conditions, MDL 12330A and KT 5720 application. After a $500 \mathrm{~ms}$ depolarizing pulse, endocytosis followed a very slow time course in the presence of MDL 12330A and KT 5720 compared with that in control conditions. (Control: black circles; MDL 12330A: blue circles; KT 5720, red circles, mean \pm SEM). 


\subsubsection{The effect of calmodulin inhibitors on endocytosis at the calyx of Held synapse}

Previous study has indicated that both rapid and slow forms of endocytosis were completely blocked by calmodulin inhibitors at the calyx of Held, indicating that calmodulin initiates all forms of endocytosis (Wu et al., 2009). The role of calmodulin was re-examined in this study by inhibiting the calmodulin function with two peptides (calmodulin binding domain (Yazawa et al., 1992) and calmodulin inhibitory peptide (Torok and Trentham, 1994)), both at $20 \mu \mathrm{M}$. The concentration of the peptide was similar to that in previous studies (Sakaba and Neher, 2001a; Wu et al., 2009), so it should be sufficient to block the calmodulin function ( $\mathrm{Ki}$ of $\mathrm{nM}$ order). In contrast to $\mathrm{Wu}$ et al. (2009), however, the effect of calmodulin inhibitory peptide on endocytosis in response to a $50 \mathrm{~ms}$ pulse was almost negligible (Fig. 3-13A and B, left, statistically not signifant). When a strong stimulation (500 ms) pulse was applied, membrane retrieval was significantly slowed down by the calmodulin inhibitory peptide, but endocytosis was not completely blocked. In response to a $500 \mathrm{~ms}$ pulse, fast endocytotic rate was not affected by calmodulin inhibitory peptide in our experimental condition $(145 \pm 23 \mathrm{fF} / \mathrm{s}$ for control $(\mathrm{n}=9)$ and $125 \pm 31 \mathrm{fF} / \mathrm{s}$ for calmodulin inhibitory peptide $(\mathrm{n}=6)$, respectively, $p>0.05$ ); whereas slow-form of endocytosis was significantly slowed by the peptide, the endo-rate was $36.0 \pm 7.9 \mathrm{fF} / \mathrm{s}$ for control $(\mathrm{n}=9)$ and $10.0 \pm 0.9 \mathrm{fF} / \mathrm{s}$ for calmodulin inhibitory peptide $(\mathrm{n}=6)$, respectively, $\mathrm{p}<0.01$ (also see table 3-2). Also, $100 \mu \mathrm{M}$ calmodulin inhibitory peptide was included in the presynaptic patch pipette to ensure that the concentration of the peptide was sufficiently high in the presynaptic terminal in some experiments ( 4 cells each, data not shown). The results were similar irrespective of the concentrations. Similar results have been obtained with calmodulin binding domain, another calmodulin inhibitor (Fig. 3-14). Our data are consistent with the idea that calmodulin modulates the endocytotic capacity in stimulus-dependent manner rather than initiate endocytosis, similar to the $\mathrm{Ca}^{2+}$-control of endocytotic capacity in hippocampal cultured neurons (Balaji and Ryan, 2007). 
A 0.5 mM EGTA

$50 \mathrm{~ms}$ pulse

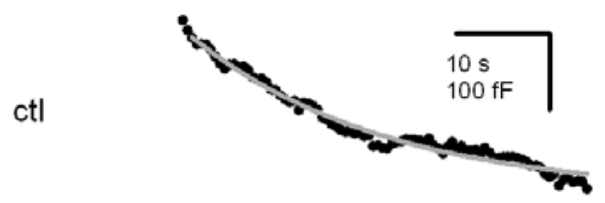

שחורות

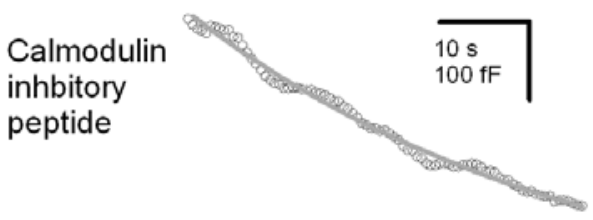

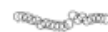

B

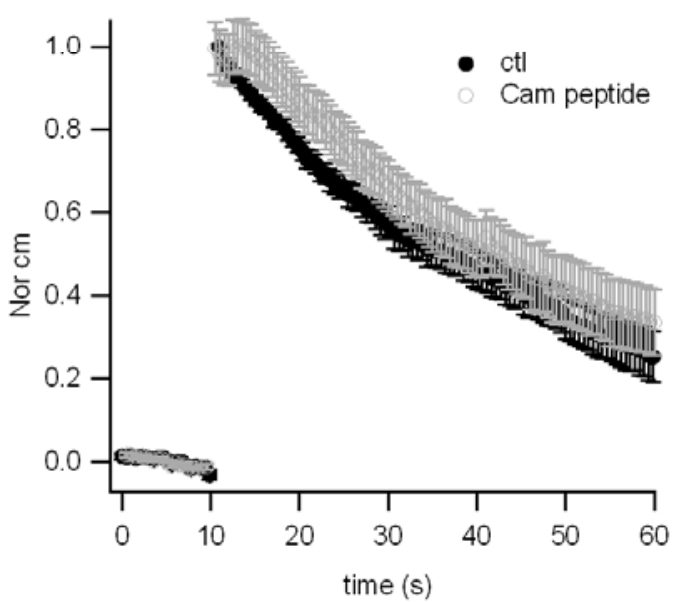

$500 \mathrm{~ms}$ pulse
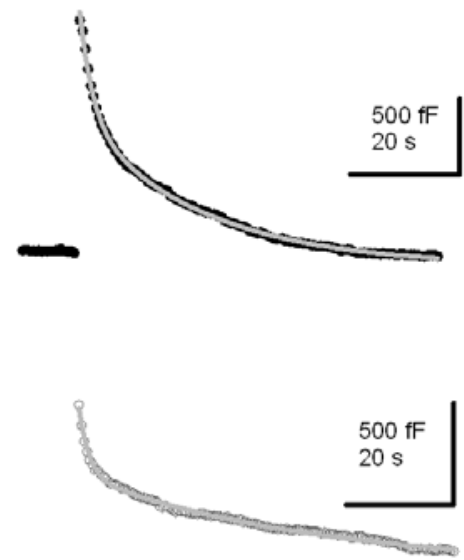

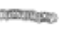

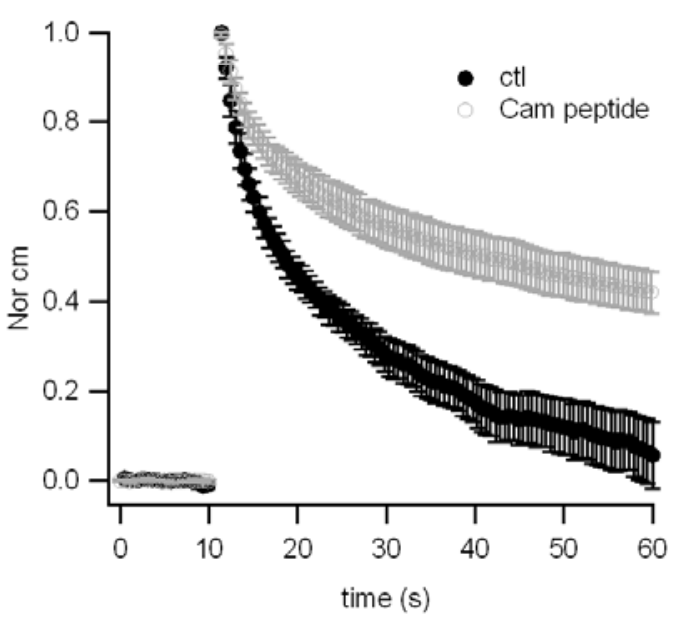

Figure 3-13: Inhibition of endocytosis rate during strong stimulation by presynaptic inclusion of calmodulin inhibitory peptide.

A: A step depolarizing pulse (from $-80 \mathrm{mV}$ to $0 \mathrm{mV}$ ) was applied to the presynaptic terminal under control condition (top) and in the presence of calmodulin inhibitory peptide (bottom) in the presynaptic patch pipette. The pipette solution also contained $0.5 \mathrm{mM}$ EGTA. The pulse duration was either 50 (left) or $500 \mathrm{~ms}$ (right). A single or a double exponential fit was used to calculate the endocytosis rates (grey lines), which are summarized in Table 3-1 and 3-2. B: The normalized time 
courses of endocytosis are plotted. Control (filled black circles) and the condition in the presence of calmodulin inhibitory peptide (grey circles).

A $50 \mathrm{~ms}$
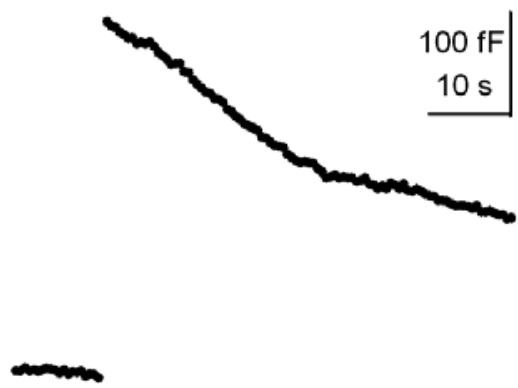

B

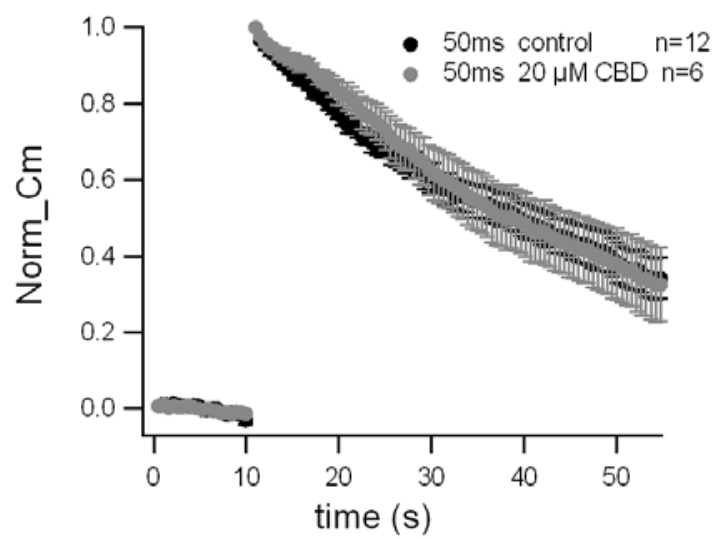

$500 \mathrm{~ms}$

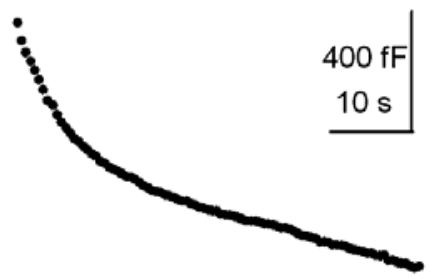

$10 \mathrm{~s}$

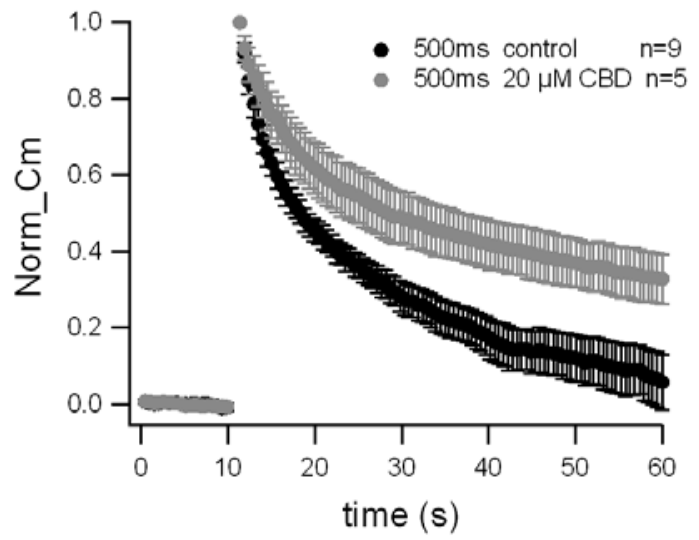

Figure 3-14: Calmodulin binding domain peptide inhibits endocytosis following a strong stimulation.

The same experiment as Fig. 3-13, but calmodulin binding domain peptide (20 $\mu \mathrm{M})$, another calmodulin inhibitor, was included into the presynaptic terminal. The peptide blocked slow endocytosis following a $500 \mathrm{~ms}$ pulse, but not a $50 \mathrm{~ms}$ pulse in the presence of the inhibitor. A shows the individual capacitance trace in response to a $50 \mathrm{~ms}$ (left) or $500 \mathrm{~ms}$ (right) pulse. $B$ shows the normalized capacitance traces. In each cell, the capacitance trace was normalized to the peak amplitude, and then the data were averaged across cells.

So far, all the experiments with endocytosis included $0.5 \mathrm{mM}$ EGTA inside the prsynaptic pipette solution, which is standard for endocytosis measurements. The buffer 
capacity with $0.5 \mathrm{mM}$ EGTA is stronger than that in the physiological condition. It is possible that we could not observe the effect of calmodulin inhibitory peptide due to the relatively strong buffer capacity. In order to observe the calmodulin effect at more physiological condition, 0.05-0.1 mM EGTA was included in the intracellular solution in the subsequent experiments. Upon a step depolarizing pulse for $20 \mathrm{~ms}$ to the terminal, calmodulin inhibitory peptide almost abolished the rate of endocytosis $(\mathrm{p}<0.01, \mathrm{n}=5$, Fig. 3-15A). A step depolarizing pulse may lead to more accumulation of Ca compared with physiological stimulation such as APs. In order to mimic physiological conditions, we applied a train of AP-like stimuli, which has not been examined previously. As shown in Fig. 3-15B, C, we did not observe an effect of calmodulin inhibitory peptide on endocytosis in response to 10 and 20 AP-like stimuli. When stronger stimulation was applied (200 stimuli), membrane retrieval was again slowed by calmodulin inhibitory peptide (Fig. 3-15D). The data indicate that calmodulin does not initiate all forms of endocytosis, but can be reconciled with $\mathrm{Wu}$ et al. by postulating that calmodulin accelerates endocytosis in an activity dependent manner. 
0.05-0.1 mM EGTA

A $20 \mathrm{~ms}$ pulse $\mathrm{ctl}$

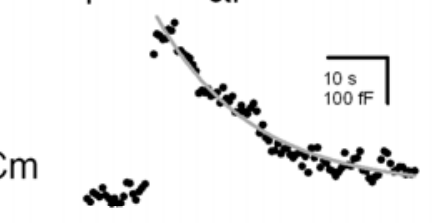

B $100 \mathrm{~Hz} \times 10$

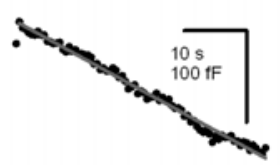

$\mathrm{Cm}$

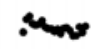

C $100 \mathrm{~Hz} \times 20$

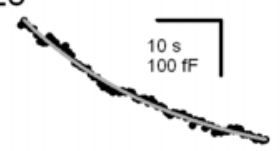

$\mathrm{Cm}$

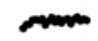

$100 \mathrm{~Hz} \times 200$

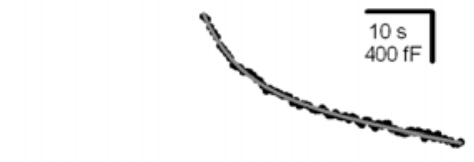

$\mathrm{Cm}$

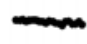

\section{Cam peptide}
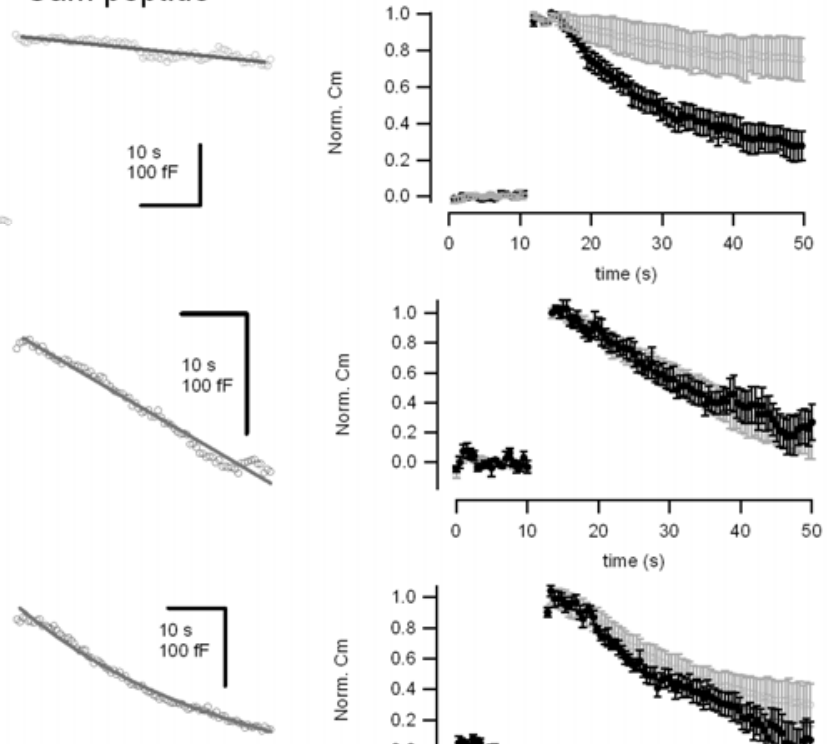

$\sin$
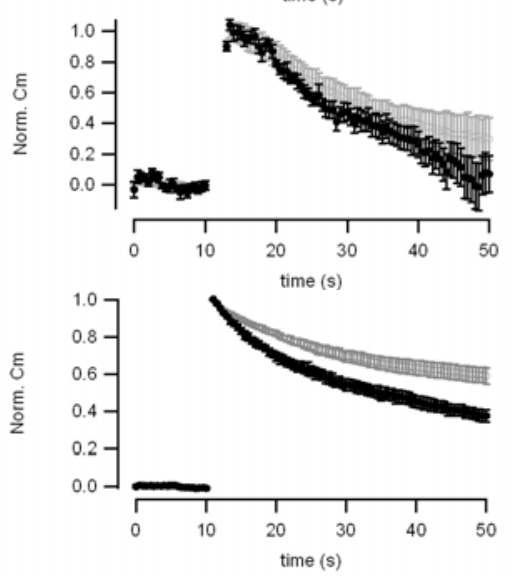

Figure 3-15: Stimulus-dependent modulation of endocytosis by calmodulin (low EGTA)

Presynaptic terminal contained 0.05-0.1 mM EGTA. In the left panels, capacitance trace under control and the condition in the presence of $20 \mu \mathrm{M}$ calmodulin inhibitory peptide in the pipette are shown. Either a step depolarizing pulse to $0 \mathrm{mV}$ for $20 \mathrm{~ms}(A)$ or a train of AP-like stimuli (depolarization to $+40 \mathrm{mV}$ for $1 \mathrm{~ms}$ at $100 \mathrm{~Hz}$, either 10 pulses $(B), 20$ pulses $(C))$ or 200 pulses $(D)$ was applied to the presynaptic terminal at the calyx of Held synapse. Grey lines indicate either an exponential or a line fit (B) to the data, which were used for calculating the endocytosis rates. In the right panels, average capacitance traces are shown. Each capacitance trace was normalized to the peak capacitance jump, before the traces were averaged across cells. Filled black circles represent the control data, whereas open grey circles represent the data in the presence of calmodulin inhibitory peptide in the patch pipette. 


\subsection{4 $\mathrm{CAMP/PKA} \mathrm{is} \mathrm{the} \mathrm{downstream} \mathrm{target} \mathrm{of} \mathrm{calmodulin} \mathrm{during} \mathrm{endocytosis}$}

The effects of MDL 12330A and KT5720 were similar to those of calmodulin inhibitors. Therefore, cAMP may work as a downstream target of calmodulin. To test this possibility, the effect of cAMP $(1 \mathrm{mM})$ was examined in the presence of calmodulin inhibitory peptide $(20 \mu \mathrm{M})$. If cAMP is the downstream target, it will reverse the effect of calmodulin inhibitor. As has been shown in Fig. 3-13, calmodulin inhibitory peptide slowed the endocytosis following a $500 \mathrm{~ms}$ pulse significantly (shown also in Fig. 3-16A). Addition of cAMP reversed the effect of calmodulin inhibitor (Fig. 3-16B), and the time course of endocytosis became similar to the control condition (Fig. 3-16C, statistic difference see table 3-2). 6-Bnz-cAMP $(300 \mu \mathrm{M})$, a selective activator of PKA (Bos, 2006), reversed the effect of calmodulin inhibitor, as expected due to the involvement of PKA (Fig. 3-15C). In contrast, 8-CPT-2 Me-cAMP, a selective agonist of Epac, had no effects ( $\mathrm{n}=4$ cells, data not shown). When a $50 \mathrm{~ms}$ pulse was applied, cAMP did not increase the rate of endocytosis significantly in the presence of calmodulin inhibitory peptide (Fig. 3-17). These results suggest that cAMP/PKA is the downstream effector of calmodulin and modulates the rate of endocytosis during strong stimulation. 
A calmodulin inhibitory peptide $20 \mu \mathrm{M}$
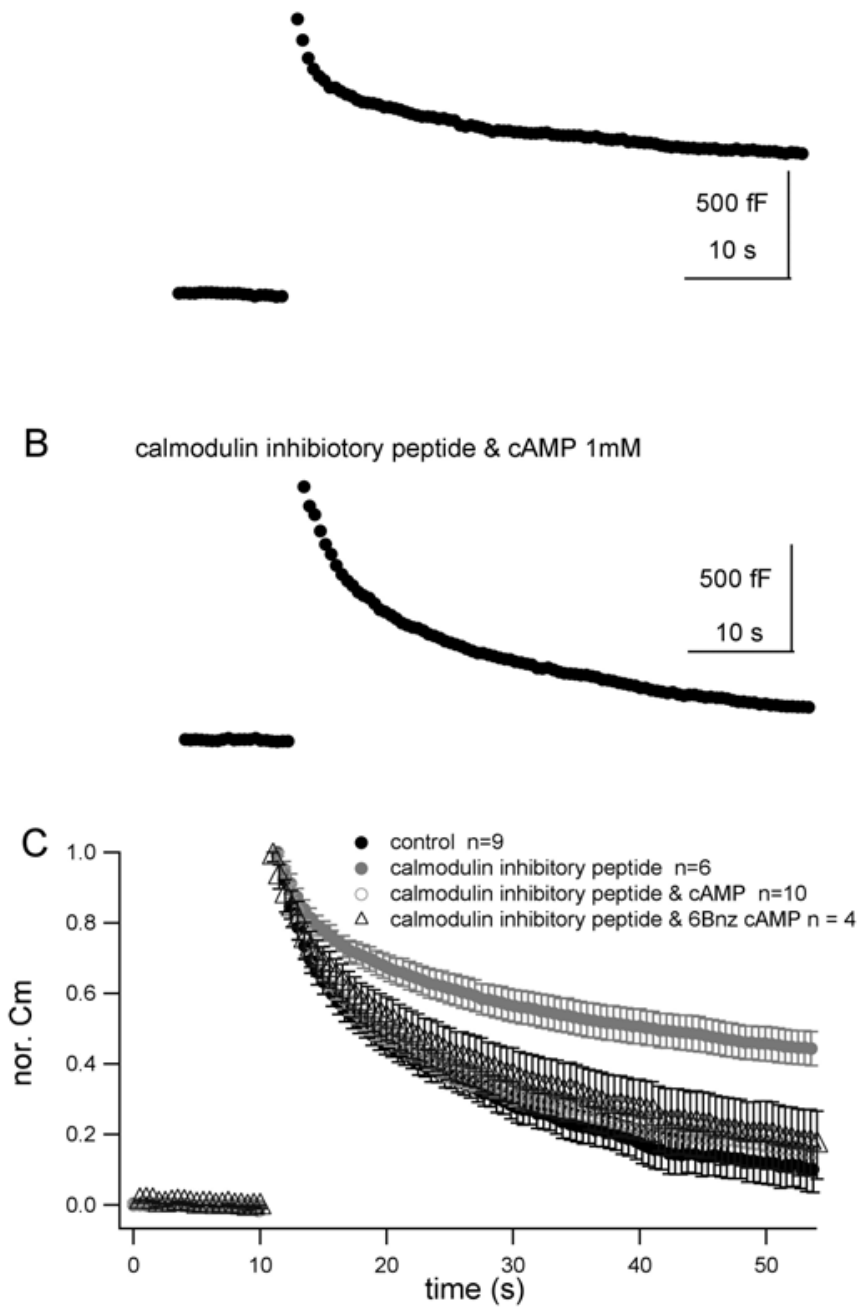

Figure 3-16: The inhibition effect of calmodulin inhibitory peptide could be reserved by addition of CAMP or PKA activator.

A, The same as Fig. 3-13, a representive capacitance trace in response to a $500 \mathrm{~ms}$ depolarizing pulse in the presence of calmodulin inhibitory peptide is shown. $B$, The same as A, but $1 \mathrm{mM}$ cAMP was further added in the pre-pipette solution. $C$, average data under control conditions (black filled circles), in the presence of calmodulin inhibitory peptide (grey filled circles), in the presence of calmodulin inhibitory peptide \& cAMP (open circles), and in the presence of calmodulin inhibitory peptide \& 6Bnz-cAMP. The normalized time courses are shown. 


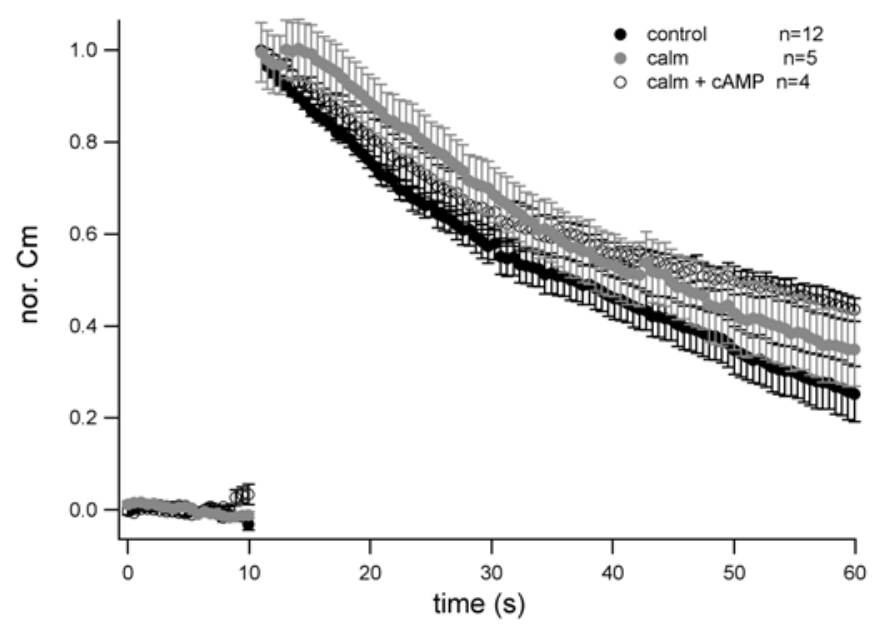

Figure 3-17: The effect of calmodulin inhibitory peptide and cAMP (a $50 \mathrm{~ms}$ pulse).

The same as Fig. 3-15, but a $50 \mathrm{~ms}$ pulse was used instead of a $500 \mathrm{~ms}$ pulse. There was no effect of cAMP in this case, when the calmodulin inhibitor was present.

Table 3-1: The summary of the endocytosis data obtained from a $50 \mathrm{~ms}$ pulse protocol

\begin{tabular}{|l|l|l|l|}
\hline Condition & $\mathrm{n}$ & Cm jump $(\mathrm{fF})$ & Endo rate $(\mathrm{fF} / \mathrm{s})$ \\
\hline Control & 12 & $379 \pm 42$ & $11.2 \pm 1.8$ \\
\hline CaM inh peptide & 10 & $344 \pm 42$ & $8.7 \pm 1.3$ \\
\hline CBD & 6 & $375 \pm 29$ & $8.6 \pm 2.1$ \\
\hline KT5720 & 5 & $306 \pm 10$ & $5.1 \pm 1.5 *$ \\
\hline Cam pep+cAMP & 4 & $461 \pm 37$ & $13.6 \pm 1.8$ \\
\hline
\end{tabular}

Statistical significance: $* \mathrm{p}<0.05$

Individual traces were fitted by an exponential, and average capacitance jumps and rates of endocytosis are shown. 
Table 3-2: The summary of the endocytosis data obtained from a $500 \mathrm{~ms}$ pulse protocol (0.5 mM EGTA in the patch pipette)

\begin{tabular}{|l|l|l|l|l|}
\hline Condition & $\mathrm{n}$ & Cm jump (fF) & Fast endo (fF/s) & Slow endo (fF/s) \\
\hline Control & 9 & $1153 \pm 116$ & $145 \pm 23$ & $36.0 \pm 7.9$ \\
\hline CaM inh peptide & 6 & $1350 \pm 118$ & $125 \pm 31$ & $10.0 \pm 0.9 *$ \\
\hline CBD & 5 & $1144 \pm 125$ & $85 \pm 13 *$ & $13.7 \pm 2.4 *$ \\
\hline KT5720 & 5 & $983 \pm 132$ & & $5.5 \pm 1.2 * *$ \\
\hline Cam pep+cAMP & 10 & $1472 \pm 90$ & $209 \pm 26$ & $38 \pm 5^{* *}$ \\
\hline
\end{tabular}

Statistical significance: $* \mathrm{p}<0.05, * * \mathrm{p}<0.01$

Note that calmodulin inhibitory peptide + cAMP with compared with calmodulin alone. Individual trace was fitted by a double exponential, and average capaciatce jumps and rates of endocytosis (fast and slow components) are shown.

\subsubsection{Calmodulin modulates synaptic vesicle replenishment and endocytosis}

\section{differently}

Endocytosis following a $50 \mathrm{~ms}$ pulse was not modulated by calmodulin inhibitors in the presence of $0.5 \mathrm{mM}$ EGTA in the presynaptic patch pipette. Under this condition, we studied the depletion and recruitment of vesicle pools by applying pairs of a $50 \mathrm{~ms}$ pulse and measuring the subsequent recovery (Hosoi et al., 2009; Sakaba and Neher, 2001a). The time-course of neurotransmitter release during a depolarizing pulse was estimated from the evoked EPSCs (Fig. 3-18A) using the deconvolution method. Transmitter release could be fitted by a double exponential function, with time-constants of 2-3 ms ( $\approx 50 \%$ of the total RRP, fast-releasing vesicles) and 10-30 ms, respectively (slowlyreleasing vesicles). When the second pulse was applied with an interval of $500 \mathrm{~ms}$, the fast component of release recovered to $60 \%$, whereas the slowly-releasing component recovered fully. The intracellular application of calmodulin inhibitory peptide blocked 
recovery of the fast component significantly $(\mathrm{p}<0.01)$ without affecting that of the slow component (Fig. 3-18B, D). Therefore, calmodulin inhibitors slowed synaptic vesicle replenishment without affecting endocytosis significantly under this condition, since the sensitivity to $\mathrm{Ca}$ buffers may differ between the two processes. Because PKA is a possible downstream target of calmodulin in endocytosis, we examined whether PKA inhibitor slowed synaptic vesicle replenishment. Application of $2 \mu \mathrm{M}$ KT5720, which affected endocytosis similar to calmodulin inhibitors, did not affect the recovery of synaptic responses during the second pulse (Fig. 3-18C, D), confirming that calmodulin/cAMP/PKA-dependent endocytosis was not related to synaptic vesicle replenishment. Taken together, we conclude that calmodulin modulates synaptic vesicle replenishment and endocytosis independently, but its regulation of synaptic vesicle replenishment cannot be entirely explained by the clearance of release sites. 


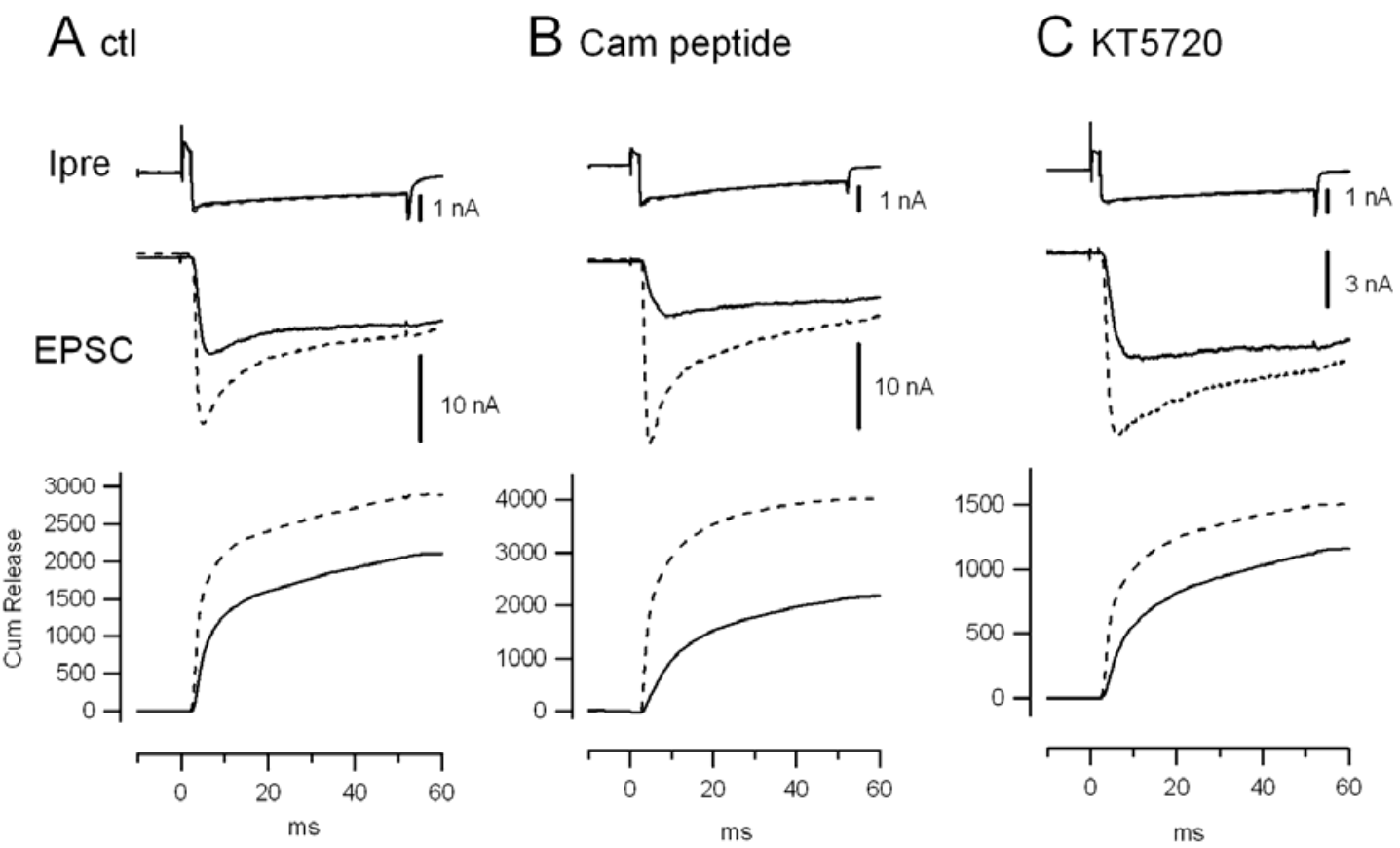

D (a)

(b)
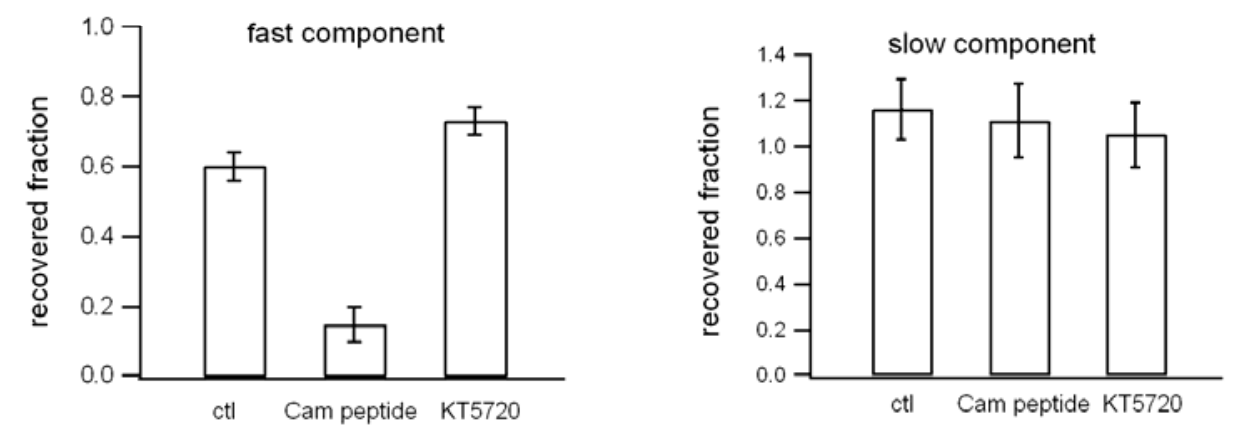

Figure 3-18: Dissociation between the calmodulin-dependent component of synaptic vesicle replenishment and calmodulin-dependent endocytosis.

A pair of the step-depolarising pulses (prepulse to $+70 \mathrm{mV}$ for $2 \mathrm{~ms}$ followed by repolarization to 0 $\mathrm{mV}$ for $50 \mathrm{~ms}$ ) was applied with an interval of $500 \mathrm{~ms}$. Dotted and solid lines indicate the data from the first and the second pulse, respectively. From top, presynaptic currents, EPSCs, and cumulative amounts of release estimated by the deconvolution method are shown. Control data $(A)$, the data obtained in the presence of calmodulin inhibitory peptide (B) or KT5720 (C) are shown. D: Recovery of the fast $(a)$ and the slow $(b)$ component of transmitter release are plotted. The data were obtained by comparing the values during the first and the second pulses. 


\section{Discussion}

The strength of a synapse can be modified by many factors. Not only do electrical activities induce short- and long-term plasticity, but hormones and second messengers also modulate synaptic strengths. In this study, the calyx of Held was chosen to study the role of second messengers in exo- and endocytosis because of its unique possibility to manipulate presynaptic function using direct patch-clamp at the terminal. The calyx of Held, a giant synapse in the auditory brainstem, has been one of the model synapses used to study the mechanisms underlying synaptic transmission and its modulation. While the calyx of Held synapse is supposed to be specialized for transmitting the auditory signals with high fidelity, synaptic properties such as the kinetics of transmitter release and shortterm plasticity such as facilitation, depression and posttetanic potentiation are similar to those at small conventional synapses. Only long-term potentiation has not been described, perhaps because the calyx of Held synapse serves as a faithful signal conveyor, rather than a signal convertor.

At the calyx of Held, two kinetically different components of transmitter release were described; one with a fast releasing time constant of 2-3 ms, while the other one with a slow releasing time constant of $20-30 \mathrm{~ms}$ in response to a step depolarizing pulse (Sakaba and Neher, 2001). The kinetic difference arises from the distance between synaptic vesicles and active zones (Wadel et al., 2007). cAMP is known as an important second messenger in the cellular signalling, and is known to potentiate neurotransmitter release in the CNS. At the calyx of Held, it has been found that cAMP affects only fastreleasing vesicles (Sakaba and Neher, 2001b); cAMP/Epac pathway, but not cAMP/PKA pathway, might be involved in the potentiation (Kaneko and Takahashi, 2004; Sakaba and Neher, 2001b). How cAMP executes its function in vesicle fusion and if cAMP has other important roles are remaining questions. In the first part of this study, I examined how cAMP modulated the intracellular $\mathrm{Ca}^{2+}$ sensitivity of transmitter release. Double patch-clamp recordings of pre- and postsynaptic compartments, $\mathrm{Ca}^{2+}$ perfusion and $\mathrm{Ca}^{2+}$ uncaging techniques were used to explore the intrinsic $\mathrm{Ca}^{2+}$ sensitivity of synaptic vesicle fusion. The elevation of intracellular cAMP concentration increased the $\mathrm{Ca}^{2+}$ sensitivity 
of vesicle fusion predominantly at lower $\left[\mathrm{Ca}^{2+}\right]_{i}$ by altering the 'willingness' of vesicle fusion $\left(l_{+}\right)$. This is similar to the effect of phorbol esters at the calyx of Held demonstrated by Lou et al. (Lou et al., 2005). However, Lou et al. did not discriminate which component of release was affected by phorbol esters. In this study, the potentiation was limited to the fast-releasing synaptic vesicles; cAMP did not affect the slowlyreleasing vesicles. In addition, the effect of cAMP in endocytosis was also investigated using membrane capacitance measurements. It was found that endogenous cAMP level was very high in the presynaptic terminal, and lowering cAMP concentration markedly slowed the rate of endocytosis under strong stimulation, while elevating of cAMP concentration only has moderately effect on endocytosis. Furthermore, calmodulin modulates the endocytosis rate rather than initiating endocytosis. During strong stimulation, the block of calmodulin function strongly slowed down the rate of slow endocytosis, but the fast endocytosis remained intact. cAMP as well as the activator of PKA (not the activator of Epac) could reverse the blocking effect of calmodulin peptides, indicating that $\mathrm{cAMP} / \mathrm{PKA}$ is the downstream target after activation of calmodulin. In conclusion, cAMP modulates synaptic function differently in different steps of the synaptic vesicle cycle (Fig. 4-1).

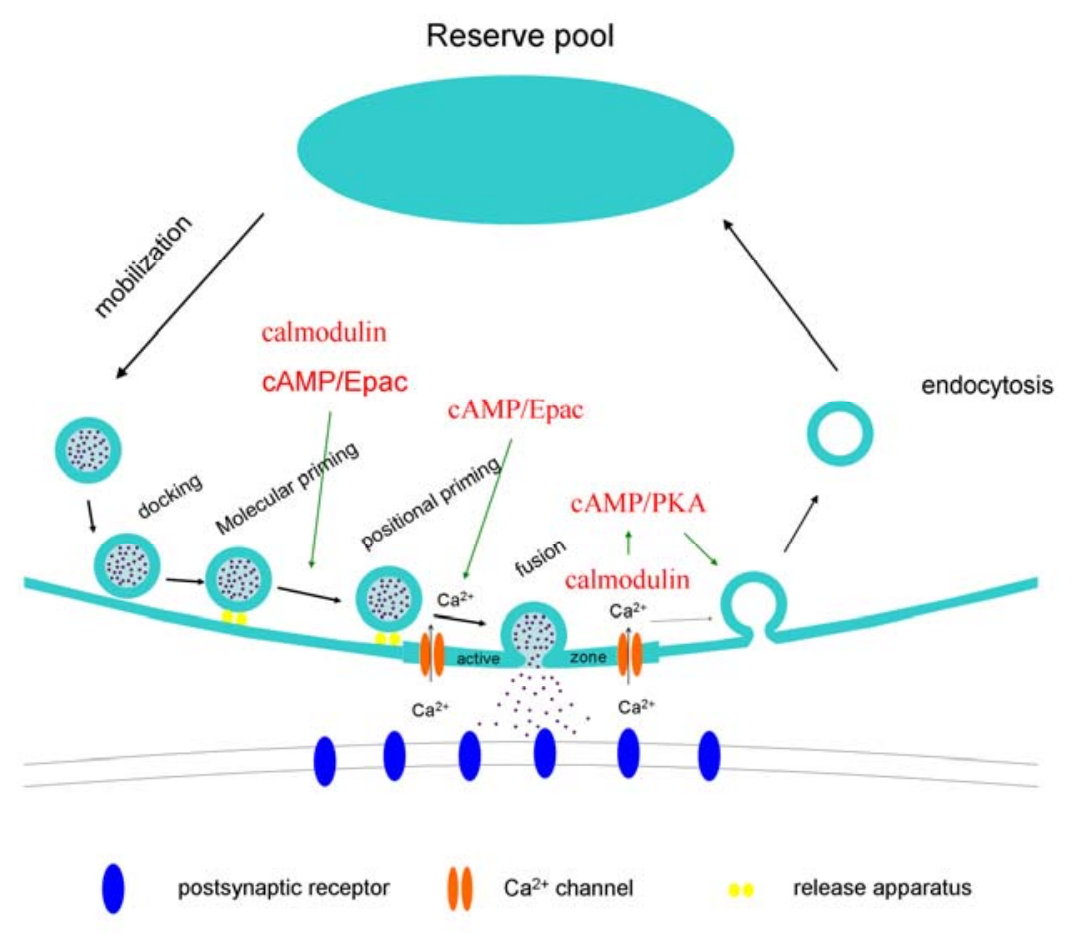


Figure 4-1: A scheme illustrating how cAMP is involved in the synaptic vesicle cycle.

cAMP participates in the process of synaptic vesicle fusion, endocytosis and recuriment of synaptic vesicles from the reserve pool to the readily releasable pool with different downstream targets.

\subsection{The potentiation of synaptic transmission by cAMP at the calyx of}

\section{Held synapse}

The potentiation of transmitter release arises from an increase in the release probability $(p)$, the number of releasable vesicles $(N)$, or both. Based on the structures and functions, different types of synapses may have different mechanisms. In the cerebellar parallel-fiber-Purkinje cell synapse (Chen and Regehr, 1997) and cultured excitatory and inhibitory hippocampal synapses (Gekel and Neher, 2008), most of the enhancement caused by cAMP arose from an increase in $p$, although small changes of $N$ could not be excluded. High frequency train stimulation is often used to estimate $N$ and $p$ (Iwasaki and Takahashi, 2001; Kaneko and Takahashi, 2004; Schneggenburger et al., 1999; Taschenberger and von Gersdorff, 2000). The idea behind this method is that, during high frequency stimulation, the RRP can be depleted within a certain number of AP-like stimuli, and the recruitment of newly synaptic vesicles takes place during the steady-state following synaptic depression. The amount of release $(N q, q$ means the average quanta size, which can be recorded during the resting period as mEPSCs) can be estimated by back-extrapolating the cumulative release from steady-state to zero time point. The initial release probability $p$ can be estimated by $N q$ divided by the amplitude of the first EPSC during the train stimulation. The merit of this method is that one can apply this technique to small synapses as well. Kaneko and Takahashi used this method to estimate the $N$ and $p$ at the calyx of Held. After application of $50 \mu \mathrm{M}$ forskolin, $N$ was increased by $179 \pm 54 \%$ on average, and $p$ was increased by $113 \pm 13 \%$ in the presense of $1 \mathrm{mM} \mathrm{Ca}^{2+}$ external solution (Kaneko and Takahashi, 2004). Under this condition, the EPSCs reached steady-state before the depletion of the vesicle pool, which might underestimate $N$. Also because of the relatively low initial $p$ under $1 \mathrm{mM} \mathrm{Ca}^{2+}$, the large potentiation of the EPSCs can be observed with forskolin. Then it turns out to 
overestimate the potentiation of $N$ by forskolin. The deconvolution method together with rapid vesicle pool depletion is another way to estimate vesicle release related parameters (see materials and methods). Using this method, one can estimate $N$, as well as $p$ and the release rates more accurately, because vesicle pool is depleted in a short time with step depolarizing pulse and the effect of synaptic vesicle recruitment is minimized. With deconvolution method, Sakaba and Neher (2001b) found that cAMP selectively increased the number of fast-releasing vesicles, whereas the slowly-releasing vesicles are not affected by cAMP. However, the situation is far from the physiological condition. In this study, I compromised between the two: In order to observe the potentiation at more physiological condition, $0.2 \mathrm{mM}$ EGTA was included in the pre-pipette solution and the external $\mathrm{Ca}^{2+}$ was maintained at $2 \mathrm{mM}$. I have used a train of AP-like stimuli, which was followed by the pool depleting pulse to ensure the depletion of RRP. Also, kyn and CTZ were used to relieve postsynaptic receptor's saturation and desensitization. During a 100 $\mathrm{Hz}$ train of stimuli, the potentiation was observed during the first 5 to 6 stimuli, which is consistent with previous study (Kaneko and Takahashi, 2004). Under my experiment condition, I found that cAMP increased $p$ to 2-fold, whereas RRP was increased only by $\sim 20 \%$, which consistent with (Sakaba and Neher, 2001b). The potentaition of $N$ was much smaller than that in Kaneko and Takahashi (2004), because they used $1 \mathrm{mM} \mathrm{Ca}^{2+}$ external solution, which was insufficient to deplete the vesicle pool in a short time, as described above.

Using prolonged depolarization and deconvolution method, the fast- and slow components of release can be separated (Sakaba and Neher, 2001). The fast-releasing vesicles are proposed to be located close to the $\mathrm{Ca}^{2+}$ channel clusters at the active zone. The slowly-releasing vesicles are located further away from $\mathrm{Ca}^{2+}$ channels (Wadel et al., 2007). Upon $\mathrm{Ca}^{2+}$ influxs through presynaptic Ca channels following an AP invasion, the fast-releasing vesicles are exposed to higher $\mathrm{Ca}^{2+}$ concentration, while the slowlyreleasing vesicles are exposed to low $\mathrm{Ca}^{2+}$ concentration. At the same time, diffusion of $\mathrm{Ca}^{2+}$ to the slowly-releasing vesicles takes more time than that to the fast releasing vesicles. The kinetics of $\mathrm{Ca}^{2+}$ diffusion depends highly on the endogeneous $\mathrm{Ca}^{2+}$ buffering system. The differences in kinetics of these two components give rise to heterogeneity in vesicle release probability under physiological conditions, which doesn't 
mean they have distinct $\mathrm{Ca}^{2+}$ sensitivities. During flash photolysis, the $\left[\mathrm{Ca}^{2+}\right]_{\mathrm{i}}$ is elevated uniformly, and all the vesicles are exposed to the same concentration of $\mathrm{Ca}^{2+}$. Under this condition, the $\mathrm{Ca}^{2+}$ sensitivity of all synaptic vesicles is assayed. In order to address whether cAMP selectively modulates the fast-releasing vesicles, I separated the fast and the slow components of release. $10-15 \mathrm{~ms}$ depolarizing pulse is sufficient to release the entire fast component, leaving only the slowly releasing vesicles. Subsequently, flash photolysis was applied to probe the intracellular $\mathrm{Ca}^{2+}$ sensitivity of the slow component. As has been demonstrated in Fig. 3-7, the slowly-releasing vesicles were not modulated by cAMP. The present data gives the intriguing possibility that the fast-releasing synaptic vesciles are not only physically coupled to the $\mathrm{Ca}^{2+}$ channel cluster, but that their $\mathrm{Ca}^{2+}$ sensitivity becomes more sensitive after activation of second messnegers, although the intrinsic $\mathrm{Ca}^{2+}$ sensitivity is very similar to that of the slowly-releasing synaptic vesicles. Priming of synaptic vesicles has two steps: molecular priming, which makes the vesicles fusion-competent, and positional priming, which positions the synaptic vesicles close to the $\mathrm{Ca}^{2+}$ channel cluster, most likely corresponding to the specific release sites within active zones (Neher and Sakaba, 2008). The fast-releasing vesicles are those that are molecularly and positionally primed whereas the slowly releasing vesicles are only molecularly primed. From Fig.3-7, we postulate that the cAMP and/or its downstream targets are located close to the $\mathrm{Ca}^{2+}$ channel cluster at the active zone and 'super primes' vesicles (Schluter et al., 2006) via the protein complex at the active zones. This superpriming of synaptic vesicles has been also postulated by a recent study at the calyx of Held (Muller et al.).

\subsection{The underlying mechanism that cAMP increases the intracellular}

\section{Ca2+ sensitivity of the fast-releasing synaptic vesicles fusion}

Synaptic vesicle fusion critically depends on the $\left[\mathrm{Ca}^{2+}\right]_{\mathrm{i}}$. So far, there has been no quantitative analysis to study how cAMP changes the $\mathrm{Ca}^{2+}$ sensitivity for vesicle fusion at the calyx of Held synapse. In this study, $\left[\mathrm{Ca}^{2+}\right]_{i}$-clamping, weak and normal flash photolysis were applied to address this issue. The $\mathrm{Ca}^{2+}$ uncaging method was used to uniformly elevating $\left[\mathrm{Ca}^{2+}\right]_{\mathrm{i}}$ in the presynaptic terminal to study the intrinsic $\mathrm{Ca}^{2+}$ 
sensitivity of vesicle fusion under the modulation by second messengers. In the range of $2-14 \mu \mathrm{M}\left[\mathrm{Ca}^{2+}\right]_{\mathrm{i}}$, the cooperativity of $\mathrm{Ca}^{2+}$ for transmitter release was high under control conditions (around 4), consistent with previous reports (Bollmann et al., 2000; Schneggenburger and Neher, 2000). Bath application of forskolin increased the $\mathrm{Ca}^{2+}$ sensitivity of vesicle fusion, but this enhancement became less prominent with an increase in $\left[\mathrm{Ca}^{2+}\right]_{\mathrm{i}}$. Accordingly, the $\mathrm{Ca}^{2+}$ cooperativity was reduced from 4 to 3 . This outcome is opposite to the cAMP effect observed at inhibitory synapses (Trudeau et al., 1998), where cooperativity was increased by elevation of cAMP. The average mEPSC frequency around basal $\left[\mathrm{Ca}^{2+}\right]_{\mathrm{i}}$ was increased 4-5-fold in the current work. In order to explain the data more quantitatively, all data sets were fitted by a simplified allosteric $\mathrm{Ca}^{2+}$-binding model (Lou et al., 2005). Compared with the conventional 5 sites $\mathrm{Ca}^{2+}$ binding model, this model adds the rate constant $l_{+}$to the conventional sequential $\mathrm{Ca}^{2+}-$ binding model (Heinemann et al., 1994; Lando and Zucker, 1994; Schneggenburger and Neher, 2000), allowing low rates of vesicle fusion to occur in the absence of bound $\mathrm{Ca}^{2+}$ to the $\mathrm{Ca}^{2+}$ sensor. According to this model, higher rates of vesicle fusion can be attained when the $\mathrm{Ca}^{2+}$ sensor is more completely occupied by $\mathrm{Ca}^{2+}$. Compared with control data, cAMP increased the vesicle fusion rate constant $l_{+} 6.7$-fold.

Other than allosteric $\mathrm{Ca}^{2+}$-binding model, two-sensor model was proposed by Sun et al. (2007), based on the experiments carried out in synaptotagmin $2 \mathrm{KO}$ mice. According to this model, fast and slow $\mathrm{Ca}^{2+}$ sensors are responsible for synchronous transmitter release and asynchronous release, respectively. And an effectivity factor $\mathrm{C}$ accounts for the change in spontaneous release in synaptotagmin-deficient synapses. We also fitted the data with this model (parameters are not shown in this thesis), According to this model, both sensors will be modulated by cAMP, but cAMP has more strong effect on the slow sensor. This is predictable since cAMP modulates vesicle fusion mainly at low $\left[\mathrm{Ca}^{2+}\right]_{\mathrm{i}}$.

The outcome of model fitting is almost the same as seen with phorbol esters, which target presynaptic protein kinase C/munc-13 signaling pathway (Hori et al., 1999; Rhee et al., 2002; Silinsky and Searl, 2003) and also increase $l_{+} 5$-fold (Lou et al., 2005). For the phorbol ester effect, the differential influence on the two sets of vesicles has not been tested directly, and the secretion model in (Lou et al., 2005) assumes that both the fastand slowly releasing vesicles are modulated equally. It is possible that the expression of 
$\mathrm{PKC} /$ munc-13 is not active zone specific, but rather more homogeneous in the terminal. Nevertheless, it remains to be tested experimentally if phorbol esters have a preferential effect on the fast-releasing vesicles, similar to the forskolin effect. Besides, cAMP and protein kinase $\mathrm{C}$ may share a similar mechanism of action on transmitter release by perhaps having the same downstream target. Application of the Epac agonist 8-pCPT-2'O-Me-cAMP (ESCA1) augmented a subsequent enhancement of evoked EPSC amplitudes by phorbol esters in cultured mouse autaptic neurons from the hippocampus, and this effect was maximal when ESCA1 application preceded the PDBu application by 3 min, indicating a downstream effect of cAMP in Epac-to-PKC signalling (Gekel and Neher, 2008). Kaneko and Takahashi (2004) showed that the forskolin- and phorbol ester effects were additive, arguing against a common target. Different type of synapses may have different mechanisms. Nevertheless, the molecular mechanism of cAMP action remains to be elucidated further.

\subsection{Calmodulin modulates the rate of endocytosis in response to strong}

\section{stimulation}

Wu et al. (2009) suggested that $\mathrm{Ca}^{2+} /$ calmodulin initiated all forms of endocytosis irrespective of stimulation patterns (Wu et al., 2009). This finding is surprising, because different forms of endocytosis are not only kinetically different, but are also supposed to be mediated through distinct molecular mechanisms. It is therefore questionable if one single mechanism can be responsible for all forms of endocytosis. We re-examined the role of calmodulin in endocytosis at the young calyx of Held synapses, and found that calmodulin facilitated endocytosis in a stimulation-dependent manner but does not initiate endocytosis. With $0.5 \mathrm{mM}$ EGTA in the pre-pipette solution, calmodulin inhibitors slowed the rate of membrane retrieval only for strong stimulations, but not for weak stimulations. This is in contrast to Wu et al. (2009). The exact reason for this discrepancy is not so clear, but it is unlikely that we could not block endocytosis sufficiently. The concentration of the peptide in most of the experiments we used was the same as in previous studies, including that of Wu et al. (2009); even higher concentration of the peptide gave the same results. Our data are rather consistent with the idea that 
$\mathrm{Ca} /$ calmodulin modulates the endocytotic capacity similar to the role of $\mathrm{Ca}^{2+}$ in hippocampal cultured neurons (Balaji et al., 2008; Balaji and Ryan, 2007): Under block of calmodulin, endocytotic rate constant increases as the amount of exocytosis increases, indicating the limited capacity of endocytosis. Under the activation of calmodulin, the rate of endocytosis increases significantly as the stimulation increases, thereby retrieving the membrane in a similar time course irrespective of stimulation patterns. It is important to note that a recent study suggested that calmodulin is not involved in endocytosis in mature calyx of Held synapses. It remains to be tested if the calmodulin-dependent process disappears with development, or else $\mathrm{Ca}^{2+}$ does not elevate high enough to activate the process due to strong expression of $\mathrm{Ca}^{2+}$ buffers in the mature animals. At more physiological condition (0.05-0.1 mM EGTA in the pipette solution), calmodulin inhibitory peptide did inhibit endocytosis after $20 \mathrm{~ms}$ depolarizaing pulse, but not after 10 or $20 \mathrm{APs}$ in $100 \mathrm{~Hz}$, which is consistent with their finding that bulk $\mathrm{Ca}^{2+}$ activates calmodulin to participate in the process of endocytosis at young calyces.

Following exocytosis, endocytosis occurs either within or at peripheral active zone is still under debate. Nevertheless, released materials at the release sites have to be cleared from the release sites to allow new synaptic vesicles to dock and become readilyreleasable. Wu et al. (2009) proposed that vesicle fusion disrupts release site structure, interfering with subsequent vesicle priming and/or fusion; $\mathrm{Ca}^{2+} /$ calmodulin may facilitate the structural recovery, clearing the release sites by endocytosis and enhancing synaptic vesicle replenishment. Our data are inconsistent with this hypothesis for several reasons. Firstly, a $50 \mathrm{~ms}$ depolarizing pulse is more than sufficient to enhance synaptic vesicle replenishment (Hosoi et al., 2007; Sakaba and Neher, 2001a). While PKA inhibitor KT5720, affected endocytosis similarly like of calmodulin inhibitors, it did not affect the recovery of synaptic responses during the second pulse, confirming that calmodulin/cAMP-dependent endocytosis was not related to synaptic vesicle replenishment. Secondly, cAMP is downstream of $\mathrm{Ca}^{2+} /$ calmodulin for modulation of endocytosis, but not for synaptic vesicle replenishment (Sakaba and Neher, 2003). For vesicle replenishment, $\mathrm{Ca}$ and cAMP act independently. This means that the underlying processes are not necessarily the same, although both synaptic vesicle replenishment and endocytosis are affected by both calmodulin and cAMP. Finally, endocytosis is 
modulated by PKA but not Epac. This is opposite for synaptic vesicle replenishment, which is modulated by Epac but not by PKA (Kaneko and Takahashi, 2004; Sakaba and Neher, 2003). Synaptic vesicle replenishment requires the bulk Ca (Hosoi et al., 2007), while endocytosis is initiated by the local Ca close to the Ca channel cluster (Hosoi et al., 2009; Wu et al., 2009). This may suggest that synaptic vesicle replenishment cannot be entirely explained by the clearance of release sites. Alternatively, one has to postulate that clearance of release sites through endocytosis and priming of synaptic vesicles are two independent processes, both of which are necessary for synaptic vesicle replenishment. Alternatively, endocytosis has to be split into an intermediate process and membrane retrieval, and the two processes are modulated by calmodulin separately. In this case, however, the time course of membrane retrieval has to be determined by local $\mathrm{Ca}^{2+}$, either at the release sites or at the endocytotic sites in the periactive zone. In the former case, the local $\mathrm{Ca}^{2+}$ has to prime the endocytotic activity in advance to membrane retrieval, because the membrane retrieval is much slower than the local $\mathrm{Ca}^{2+}$ signals. Nevertheless, calmodulin may affect synaptic vesicle replenishment independent of clearance of release sites, for example through molecular priming of synaptic vesicles through Munc13, whereas membrane retrieval can be modulated by another Ca sensor.

\section{4 cAMP/PKA is a downstream target after the activation of $\mathrm{Ca}^{2+} /$ calmodulin}

Inhibition of cAMP and PKA blocked both fast and slow forms of endocytosis (Fig. 3-12). Endocytosis is not completely abolished by the inhibitors, which indicates that a mechanism other than the cAMP/PKA-dependent process may be involved during strong stimulation. The endocytosis in response to a short depolarizing pulse was not strongly affected by the AC inhibitor or the PKA inhibitor. This suggests that cAMP/PKA can only be activated by massive stimulation. ACs were proposed to have 12 transmembrane domains which share some properties with some voltage-sensitive ion channels (Gao and Gilman, 1991; Krupinski et al., 1989). In cultured cerebellar and hippocampus neurons, AC activity is synergistically stimulated by depolarization (Cooper et al., 1998; Reddy et al., 1995). Although direct demonstration of $\mathrm{Ca}^{2+}$ stimulation of $\mathrm{AC}$ is lacking at the 
calyx of Held, it is possible that during a long depolarizing pulse, ACs are activated in our experimental conditions, leading to the activation of PKA. In mammals, at least nine isoforms of $\mathrm{ACs}$ were characterized; $\mathrm{AC} 1, \mathrm{AC} 3$ and $\mathrm{AC} 8$ are $\mathrm{Ca}^{2+} /$ calmodulin dependent enzymes (Mons and Cooper, 1994; Xia et al., 1993; Xia et al., 1992). AC1 and AC8 are expressed in different regions of the brain; specific localization may suggest specific functions (Defer et al., 2000). $\mathrm{Ca}^{2+}$ can either stimulate or inhibit ACs, depending on the specific AC isoforms. Usually several AC isoforms are co-expressed in the same type of cells, mapping a complex system to modulate different cell functions, such as synaptic plasticity, differentiation, development and so on (Defer et al., 2000). It is unknown at present which isoforms are expressed at calyx of Held. Nevertheless, we suggest that cAMP/PKA acts downstream of calmodulin. This means that the slow mode of endocytosis observed at the calyx of Held is different from the calmodulin-sensitive component of endocytosis observed at other synapses, where calmodulin modulates endocytosis directly or else via calcineurin (Cousin and Robinson, 2000). Calmodulin sensitive slow form of endocytosis can only be induced with massive stimulation, and in our experimental condition, it seems that the fast form of endocytosis is calmodulin insensitive. However with the inhibitors of $\mathrm{AC}$ and PKA, both forms of endocytosis are inhibited. We don't know the exact reason for this discrepancy. It is possible that some PKA activity remains after inhibition of calmodulin. PKA has been implicated in modulating somatic endocytosis (Zhang et al., 2004). Zhang et al. characterized a rapid form of endocytosis which is calcium- and dynamin-independent, and this form is controlled by PKA-dependent phosphorylation in dorsal root ganglion neurons in the peripheral neural system. Although the downsteam target of PKA remains to be identified, this is the first study indicating the role of PKA in endocytosis in nerve terminal. Because fast endocytosis may potentially enhance synaptic vesicle cycling, it may have some relevance in short- and long term presynaptic plasticity, which depends on the activation of calmodulin and PKA. 


\section{Summary}

Short-term plasticity is a fundamental property of all synapses but its underlying mechanisms remain to be elucidated. In this study, the functional role of the well-known second messenger, cAMP, was studied at the calyx of Held synapse, a giant glutamatergic synapse in the auditory brainstem. By combining electrophysiological recordings of the pre- and postsynaptic compartments with $\mathrm{Ca}^{2+}$ imaging and $\mathrm{Ca}^{2+}$ uncaging, it was found that elevation of cAMP concentration increased intracellular $\mathrm{Ca}^{2+}$ sensitivity for transmitter release, especially at lower $\mathrm{Ca}^{2+}$ concentrations. The change in $\mathrm{Ca}^{2+}$ sensitivity was limited to the fast-releasing synaptic vesicles, but not the slowlyreleasing vesicles. Fitting of the data using a simplified allosteric model indicated that cAMP increased the fusion 'willingness', thereby facilitating transmitter release. It was suggested that synaptic vesicles have to be positionally primed to the release sites close to the $\mathrm{Ca}^{2+}$ channel cluster for cAMP to modulate intracellular $\mathrm{Ca}^{2+}$ sensitivity.

In addition to exocytosis, I have used time-resolved capacitance measurement to examine whether cAMP modulates endocytosis. It was found that lowering the concentration of cAMP in the terminal as well as inhibition of PKA activity slowed down the rate of endocytosis during strong stimulation, indicating the involvement of cAMP/PKA in endocytosis. Calmodulin blockers slowed the rate of slow form of endocytosis following strong, but not weak presynaptic stimulation. As a result, the time course of endocytosis got progressively slower as the amounts of endocytosis increased. This suggested that calmodulin facilitated but not initiated endocytosis in an activitydependent manner at the calyx of Held synapse. Finally, we suggested that cAMP/PKA might be the downstream target of calmodulin.

In conclusion, cAMP is involved in the whole process of synaptic vesicle recycling. It modulates exocytosis, endocytosis and synaptic vesicle recruitment differently: For synaptic vesicle fusion, cAMP/Epac modulates the intracellular $\mathrm{Ca}^{2+}$ sensitivity for fast transmitter release. For endocytosis, $\mathrm{Ca}^{2+} /$ calmodulin-cAMP/PKA is involved in synaptic vesicle retrieval. For vesicle recruitment, $\mathrm{Ca}^{2+} /$ calmodulin and $\mathrm{cAMP} / \mathrm{Epac}$ are also known to be important, but they work independently. 


\section{Bibliography}

Balaji, J., and Ryan, T.A. (2007). Single-vesicle imaging reveals that synaptic vesicle exocytosis and endocytosis are coupled by a single stochastic mode. Proceedings of the National Academy of Sciences of the United States of America 104, 20576-20581.

Bergsman, J.B., De Camilli, P., and McCormick, D.A. (2004). Multiple large inputs to principal cells in the mouse medial nucleus of the trapezoid body. Journal of neurophysiology 92, 545-552.

Beutner, D., Voets, T., Neher, E., and Moser, T. (2001). Calcium dependence of exocytosis and endocytosis at the cochlear inner hair cell afferent synapse. Neuron 29, 681-690.

Bollmann, J.H., Sakmann, B., and Borst, J.G. (2000). Calcium sensitivity of glutamate release in a calyx-type terminal. Science (New York, N.Y 289, 953-957.

Bos, J.L. (2006). Epac proteins: multi-purpose cAMP targets. Trends in biochemical sciences $31,680-686$.

Burgoyne, R.D., and Clague, M.J. (2003). Calcium and calmodulin in membrane fusion. Biochimica et biophysica acta 1641, 137-143.

Burgoyne, R.D., O'Callaghan, D.W., Hasdemir, B., Haynes, L.P., and Tepikin, A.V. (2004). Neuronal Ca2+-sensor proteins: multitalented regulators of neuronal function. Trends in neurosciences 27, 203-209.

Chen, C., and Regehr, W.G. (1997). The mechanism of cAMP-mediated enhancement at a cerebellar synapse. J Neurosci 17, 8687-8694.

Colbran, R.J. (2004). Protein phosphatases and calcium/calmodulin-dependent protein kinase II-dependent synaptic plasticity. J Neurosci 24, 8404-8409.

Cousin, M.A. (2009). Activity-dependent bulk synaptic vesicle endocytosis--a fast, high capacity membrane retrieval mechanism. Molecular neurobiology 39, 185-189.

de Lange, R.P., de Roos, A.D., and Borst, J.G. (2003). Two modes of vesicle recycling in the rat calyx of Held. J Neurosci 23, 10164-10173. 
Defer, N., Best-Belpomme, M., and Hanoune, J. (2000). Tissue specificity and physiological relevance of various isoforms of adenylyl cyclase. Am J Physiol Renal Physiol 279, F400-416.

Dodge, F.A., Jr., and Rahamimoff, R. (1967). Co-operative action a calcium ions in transmitter release at the neuromuscular junction. The Journal of physiology 193, 419432.

Edwards, F.A., Konnerth, A., Sakmann, B., and Takahashi, T. (1989). A thin slice preparation for patch clamp recordings from neurones of the mammalian central nervous system. Pflugers Arch 414, 600-612.

Fesce, R., Grohovaz, F., Valtorta, F., and Meldolesi, J. (1994). Neurotransmitter release: fusion or 'kiss-and-run'? Trends in cell biology 4, 1-4.

Forsythe, I.D., and Barnes-Davies, M. (1993). The binaural auditory pathway: excitatory amino acid receptors mediate dual timecourse excitatory postsynaptic currents in the rat medial nucleus of the trapezoid body. Proceedings 251, 151-157.

Heidelberger, R., Heinemann, C., Neher, E., and Matthews, G. (1994). Calcium dependence of the rate of exocytosis in a synaptic terminal. Nature 371, 513-515.

Heinemann, C., Chow, R.H., Neher, E., and Zucker, R.S. (1994). Kinetics of the secretory response in bovine chromaffin cells following flash photolysis of caged $\mathrm{Ca} 2+$. Biophysical journal 67, 2546-2557.

Holt, M., Cooke, A., Wu, M.M., and Lagnado, L. (2003). Bulk membrane retrieval in the synaptic terminal of retinal bipolar cells. J Neurosci 23, 1329-1339.

Joris, P.X., and Yin, T.C. (1998). Envelope coding in the lateral superior olive. III. Comparison with afferent pathways. Journal of neurophysiology 79, 253-269.

Jurado, L.A., Chockalingam, P.S., and Jarrett, H.W. (1999). Apocalmodulin. Physiological reviews 79, 661-682.

Kandler, K., and Friauf, E. (1993). Pre- and postnatal development of efferent connections of the cochlear nucleus in the rat. The Journal of comparative neurology 328 , 161-184.

Lindau, M., and Neher, E. (1988). Patch-clamp techniques for time-resolved capacitance measurements in single cells. Pflugers Arch 411, 137-146. 
Llinas, R., Sugimori, M., and Silver, R.B. (1995). The concept of calcium concentration microdomains in synaptic transmission. Neuropharmacology 34, 1443-1451.

Miller, T.M., and Heuser, J.E. (1984). Endocytosis of synaptic vesicle membrane at the frog neuromuscular junction. The Journal of cell biology 98, 685-698.

Muller, M., Goutman, J.D., Kochubey, O., and Schneggenburger, R. Interaction between facilitation and depression at a large CNS synapse reveals mechanisms of short-term plasticity. J Neurosci 30, 2007-2016.

Neher, E., and Sakaba, T. (2001). Combining deconvolution and noise analysis for the estimation of transmitter release rates at the calyx of held. J Neurosci 21, 444-461.

Neher, E., and Sakaba, T. (2008). Multiple roles of calcium ions in the regulation of neurotransmitter release. Neuron 59, 861-872.

Richards, D.A., Guatimosim, C., and Betz, W.J. (2000). Two endocytic recycling routes selectively fill two vesicle pools in frog motor nerve terminals. Neuron 27, 551-559.

Royle, S.J., and Lagnado, L. (2003). Endocytosis at the synaptic terminal. The Journal of physiology 553, 345-355.

Sakaba, T. (2006). Roles of the fast-releasing and the slowly releasing vesicles in synaptic transmission at the calyx of held. J Neurosci 26, 5863-5871.

Sakaba, T., and Neher, E. (2001). Calmodulin mediates rapid recruitment of fastreleasing synaptic vesicles at a calyx-type synapse. Neuron 32, 1119-1131.

Satzler, K., Sohl, L.F., Bollmann, J.H., Borst, J.G., Frotscher, M., Sakmann, B., and Lubke, J.H. (2002). Three-dimensional reconstruction of a calyx of Held and its postsynaptic principal neuron in the medial nucleus of the trapezoid body. J Neurosci 22, 10567-10579.

Schluter, O.M., Basu, J., Sudhof, T.C., and Rosenmund, C. (2006). Rab3 superprimes synaptic vesicles for release: implications for short-term synaptic plasticity. J Neurosci 26, 1239-1246.

Schneggenburger, R., and Forsythe, I.D. (2006). The calyx of Held. Cell and tissue research 326, 311-337.

Schneggenburger, R., and Neher, E. (2000). Intracellular calcium dependence of transmitter release rates at a fast central synapse. Nature 406, 889-893. 
Sun, J., Pang, Z.P., Qin, D., Fahim, A.T., Adachi, R., and Sudhof, T.C. (2007). A dual$\mathrm{Ca} 2+$-sensor model for neurotransmitter release in a central synapse. Nature 450, 676682.

Taschenberger, H., and von Gersdorff, H. (2000). Fine-tuning an auditory synapse for speed and fidelity: developmental changes in presynaptic waveform, EPSC kinetics, and synaptic plasticity. J Neurosci 20, 9162-9173.

Torok, K., and Trentham, D.R. (1994). Mechanism of 2-chloro-(epsilon-amino-Lys75)[6-[4-(N,N- diethylamino)phenyl]-1,3,5-triazin-4-yl]calmodulin interactions with smooth muscle myosin light chain kinase and derived peptides. Biochemistry 33, 12807-12820.

von Gersdorff, H., and Borst, J.G. (2002). Short-term plasticity at the calyx of held. Nature reviews 3, 53-64.

von Gersdorff, H., and Matthews, G. (1994). Inhibition of endocytosis by elevated internal calcium in a synaptic terminal. Nature 370, 652-655.

Wayman, G.A., Lee, Y.S., Tokumitsu, H., Silva, A.J., and Soderling, T.R. (2008). Calmodulin-kinases: modulators of neuronal development and plasticity. Neuron 59, 914931.

Wu, L.G., Ryan, T.A., and Lagnado, L. (2007). Modes of vesicle retrieval at ribbon synapses, calyx-type synapses, and small central synapses. J Neurosci 27, 11793-11802.

$\mathrm{Wu}, \mathrm{W}$., and Wu, L.G. (2007). Rapid bulk endocytosis and its kinetics of fission pore closure at a central synapse. Proceedings of the National Academy of Sciences of the United States of America 104, 10234-10239.

Wu, W., Xu, J., Wu, X.S., and Wu, L.G. (2005). Activity-dependent acceleration of endocytosis at a central synapse. J Neurosci 25, 11676-11683.

$\mathrm{Wu}, \mathrm{X} . \mathrm{S}$., and $\mathrm{Wu}$, L.G. (2009). Rapid endocytosis does not recycle vesicles within the readily releasable pool. J Neurosci 29, 11038-11042.

Yazawa, M., Vorherr, T., James, P., Carafoli, E., and Yagi, K. (1992). Binding of calcium by calmodulin: influence of the calmodulin binding domain of the plasma membrane calcium pump. Biochemistry 31, 3171-3176.

Zhang, C., Xiong, W., Zheng, H., Wang, L., Lu, B., and Zhou, Z. (2004). Calcium- and dynamin-independent endocytosis in dorsal root ganglion neurons. Neuron 42, 225-236. 
Zucker, R.S. (1999). Calcium- and activity-dependent synaptic plasticity. Current opinion in neurobiology 9, 305-313.

Zucker, R.S., and Regehr, W.G. (2002). Short-term synaptic plasticity. Annual review of physiology 64, 355-405. 


\section{Abbreviations}

AC adenylate cyclase

AMPA $\alpha$-amino-3-hydroxy-5-methyl-4-isoxazolepropionic acid

AP action potential

aVCN anterior ventral cochlear nucleus

$\mathrm{AZ} \quad$ active zone

cAMP cyclic adenosine monophosphate

CNS central nervous system

CTZ cyclothiazide

D-AP5 D(-)-2-amino-5-phosphonopentanoic acid

DMN DM-nitrophen

EGTA ethylene glycol tetraacetic acid

Epac exchange protein directly activated by cAMP

EPSC excitatory postsynaptic current

GBC globular bushy cell

GEF guanine nucleotide exchange factor

Kyn kynurenic acid

LSO lateral superior olive

mEPSC miniature excitatory postsynaptic current

MNTB medial nucleus of the trapezoid body

$N \quad$ the number of releasable vesicles

NMDA N-methyl-D-aspartate

$p \quad$ release probability

PKA protein kinase A

PPT Patcher's Power Tools

RRP readily releasable pool

SEM standard error of the mean

Syt synaptotagmin

TEA-Cl tetraethylammonium chloride 
TTX tetrodotoxin

UV ultraviolet

8-pCPT-2'-O-Me-cAMP 8-(Chlorophenylthio)-2'-O-methyl-cAMP

$\gamma$-DGG $\gamma$-D-glutamylglycine 


\section{Acknowledgment}

First and foremost, I'm heartily thankful to my supervisor Dr Takeshi Sakaba for giving me the opportunity to work in such a wonderful lab, for his patience, encouragement and tremendous support to my $\mathrm{PhD}$ project and thesis accomplishment. He is armed with immense knowledge, no matter in science, music, geography or history, and one of the cleverest people I'v ever met in my life.

Secondly, I'd like to give my grateful thanks to the members of my $\mathrm{PhD}$ committee, Prof. Tobias Moser and Prof. Andreas Stumpner for their suggestions and continuous support to my $\mathrm{PhD}$ project in the last three and half years.

Thanks to all the members in biophysics of synaptic transmission group, Dr. Nobutake Hosoi, for his help in experiments and data analysis at the beginning of my study; Dr. Kristian Wadel and Jin Bao helped me to set down my life in Göttingen; and also Chao-Hua Huang for sharing all the news in and outside of the lab.

My sincere thanks to Prof. Erwin Neher, for his comments on my project and manuscripts. My special thanks to Irmgard Barteczko, for her helps in life and administerial stuffs. I thank Raunak Sinha, for all his kindly helps in correcting the English for my manuscript and thesis. I thank Dr. Holger Taschenberger to provide nice macros; also I'd like to give my numerous thanks to Sigrid Schmidt, Frank Würriehausen and Frank Köhne for their excellent technical assistances. My thanks to all my colleagues, Kun-Han Lin, Yunfeng Hua, Zuxin Chen, Meike Pedersen, Samuel Young, Dirk Reuter, Dmitri Bibitchkov, Ina-Maria Herfort... for the great time I had in our department, it's a memorable experience to work in such a friendly and freely atmosphere.

I also thank all my friends in Goettingen, for sharing their time and happiness with me. Finally, I owe special gratitude to my family for unconditional support, especial blessing to my dear grandfather in another world. 


\section{Curriculum Vitae}

\section{Personal Data}

Name: $\quad$ Lijun Yao

Gender: $\quad$ Female

Date of birth: $\quad 27.10 .1979$

Place of birth: Hangzhou, China

\section{Education}

1999-2004: $\quad$ Medical Degree. in Basic Medical Sciences.

Zhejiang University, Hangzhou, China

2003-2004: Internship in biophysics group

Institute of Neuroscience, Chinese Academy of Sciences, Shanghai

2004-2006: $\quad$ M. S. in Neurobiology.

Zhejiang University, Hangzhou, China

Institute of Molecular Medicine, Peking University, Beijing

2007-2010: $\quad$ PhD student in the research group for biophysics of synaptic

transmission at the Max Planck Institute for Biophysical Chemistry,

Göttingen, Germany

Sensory and Motor Neuroscience program in the Göttingen graduate school for neurosciences and molecular biosciences (GGNB) Georg August-University Göttingen, Germany 


\section{Publication list}

1. Yao L and Sakaba T. (2010). cAMP modulates intracellular $\mathrm{Ca}^{2+}$ sensitivity of fastreleasing synaptic vesicles at the calyx of Held synapse. Journal of Neurophysiology, 104(6):3250-60.

2. Yao L and Sakaba T. Activity-dependent modulation of endocytosis by calmodulin at the calyx of Held, submitted.

3. Yao L, Wang G, Ouyang $\mathrm{K}$ et al. (2006). $\mathrm{Ca}^{2+}$ sparks and $\mathrm{Ca}^{2+}$ glows in superior cervical ganglion neurons. Acta Pharmacoligica Sinica. 27(7):848-852

4. Yao J, Chen X, Li H, Zhou Y, Yao L et al. (2005). Bmp09, a "longchain" scorpion peptide blocker of BK channels. The journal of Biological Chemistry 280(15):1481914828

5. Yao L, Zhou S, Xia Q (2004). The effects of metabotropic glutamate receptors in synaptic plasticity. Progress in physiological Sciences. Review, 35(1):73-76 